\title{
Modulation of aging process by diet and crosslinking inhibitor and their effects on production in poultry
}

\author{
Muhammad Iqbal \\ West Virginia University
}

Follow this and additional works at: https://researchrepository.wvu.edu/etd

\section{Recommended Citation}

Iqbal, Muhammad, "Modulation of aging process by diet and crosslinking inhibitor and their effects on production in poultry" (1998). Graduate Theses, Dissertations, and Problem Reports. 927.

https://researchrepository.wvu.edu/etd/927

This Dissertation is protected by copyright and/or related rights. It has been brought to you by the The Research Repository @ WVU with permission from the rights-holder(s). You are free to use this Dissertation in any way that is permitted by the copyright and related rights legislation that applies to your use. For other uses you must obtain permission from the rights-holder(s) directly, unless additional rights are indicated by a Creative Commons license in the record and/ or on the work itself. This Dissertation has been accepted for inclusion in WVU Graduate Theses, Dissertations, and Problem Reports collection by an authorized administrator of The Research Repository @ WVU.

For more information, please contact researchrepository@mail.wvu.edu. 


\title{
Modulation of Aging Process by Diet and Crosslinking Inhibitor and their Effects on Production in Poultry
}

\author{
Muhammad Iqbal, D.V.M., M.Sc. (Hons) \\ Dissertation submitted to the Faculty of the College of \\ Agriculture, Forestry and Consumer Sciences \\ West Virginia University \\ in the partial fulfillment of the requirements for the degree of \\ Doctor in Philosophy \\ H. Klandorf, Chair \\ R. A. Dailey \\ P. Keeting \\ P. B. Kenney \\ J. Killefer \\ R. A. Peterson
}

September 22, 1998

Morgantown, West Virginia

Keyword: Aging, Aminoguanidine, Diet restriction, Pentosidine, Meat quality, Reproduction 


\section{Modulation of Aging Process by Diet and Crosslinking Inhibitor and their Effects on Production in Poultry Muhammad Iqbal}

(Abstract)

The factors which influence the age-related decline in production performance and increases in meat toughness in broiler breeder hens are poorly understood. We have hypothesized that the elevated plasma concentration of glucose contributes to the nonenzymatic attachment of glucose to proteins, and generates glycoxidation crosslinks in tissues which can ultimately impair function, is responsible for loss of production. One such crosslink, pentosidine, is an established indicator of both glycative and oxidative tissue damage in mammals. Specifically, the role of diet restriction (DR) and a nucleophilic hydrazine compound, aminoguanidine (AG), a crosslinking inhibitor, were examined on tissue crosslinking with the view towards both extending the production period and improving carcass quality of broiler breeder hens. Additionally, isolation of pentosidine from skin and tendons of hens and their relationship with tendon breaking strength (TBS), time (TBT) and meat tenderness (SF) were carried out. Chicks were randomly divided into four dietary groups from eight to 125 wks after hatch: ad libitum (AL); diet restricted (DR, fed 60\% of ad libitum); AL and DR groups supplemented with $400 \mathrm{ppm}$ AG (1.35 mg/kg BW/day) each (AL+ AG and DR+AG respectively).

The results showed that pentosidine was present in the skin $\left(\mathrm{P}_{\mathrm{s}}\right)$ and tendon $\left(\mathrm{P}_{\mathrm{t}}\right)$ of the broiler breeder hens, and that there was an age-related, linear increase in its concentration $\left(p<0.001, r^{2}\right.$ $=0.94)$ for skin and $\left(p<0.0001 ; r^{2}=0.79\right)$ for tendon in AL-fed hens. DR and supplementation of AG with AL-fed hens (AL+AG) retarded $\mathrm{P}_{\mathrm{s}}(p<0.001 ; p<0.001)$ and $\mathrm{P}_{\mathrm{t}}(p<0.001 ; p<0.01)$ respectively as compared to AL-fed hens. Concurrently, there was also an age-related parallel increase in TBS $\left(p<0.0001 ; r^{2}=0.56\right)$, in TBT $\left(p<0.0001 ; r^{2}=0.67\right)$, and in shear force (SF) $\left(p<0.0001 ; r^{2}=0.95\right)$

in AL-fed hens. Similarly DR significantly retarded the overall TBS $(p<0.01)$ TBT $(p<0.02)$, and SF $(p<0.0001)$ values as compared to AL-fed hens over the period of this study. Supplementation of AL-fed hens with AG (AL+AG group) 
significantly retarded the concentration of $\mathrm{P}_{t}, \mathrm{P}_{\mathrm{s}}$ and $\mathrm{SF}$ values and was similar to those of the DR group. Although, supplementation of DR with AG $(D R+A G)$ retarded the rate of accumulation of $\mathrm{P}_{\mathrm{s}}(p<0.001)$ over the laying cycle, the reduction in $\mathrm{P}_{\mathrm{s}}$ did not significantly affect the mean production. It is therefore concluded that the rate of $\mathrm{P}_{\mathrm{s}}$ and $\mathrm{P}_{\mathrm{t}}$ accumulation and the age-related increase in meat toughness can be retarded by either DR or AG. $\mathrm{P}_{\mathrm{s}}$ can also be used as a predicator of age-related changes in meat. The same approach has the potential to be initiated in other animal production systems. Because of the linear, age-related increase in the concentrations of tissue pentosidine, it can be used as biomarker for the study of aging. 


\section{Dedication}

To my beloved parents (late) whose inspirations

lead me to pursue this study

and

brother, ljaz Ahmad (Late) 


\section{Acknowledgments}

First of all I am grateful to Almighty Allah who gave me courage, health and opportunity to accomplish the requirements for this degree.

I would express my sincerest appreciation to my graduate advisor, Dr. Hillar Klandorf, for his leadership, advice and assistance during the course of this research. Special thanks are due to my graduate advisory committee for its valuable and thoughtful contributions for the improvement of my research projects especially by Drs. P. B. Kenney and John Killefer. l acknowledge the useful inputs of Drs. Dailey and Peterson as well. I also appreciate Dr. Townsend's assistance in statistical analyses.

I acknowledge the valuable contribution of Mr. Nabil Al-Humadi (NIOSH), in modification and standardization of HPLC procedure for pentosidine estimation. I also acknowledge the ever-ready and generous helps of Sarah Holt, Diana Keller and Susan Slider. Farm crew also deserves my appreciation especially, Bill Jones, Fred, Miller, Gordon and Glen for formulating and mixing of feed and their help in talking care of my experimental birds.

I would also like to appreciate the help of my fellow graduate students in managing and slaughtering the experimental birds over the period of time, especially Hakan Kocamis, Jon Preggi, Christine Dezert and Dinesh Rathore.

Last but not least, I appreciate the support and encouragement of my wife, Yasmin, during my stay at WW as a graduate student. I appreciate the patience of my daughters' Tahreem and Farheen, and son, Asim for my long absences from home.

labal Muhammad

Morgantown, W 


\section{TABLE OF CONTENTS}

TITLE PAGE

ABSTRACT

DEDICATION iv

ACKNOWLEDGMENTS v v

TABLE OF CONTENTS vi

LIST OF TABLE AND FIGURES ix

LIST OF ABBREVIATIONS X xi

CHAPTER

Introduction 1

Objectives 4

\section{CHAPTER 2}

Review of literature $\quad 5$

What is aging $\quad 5$

Bases of aging hypotheses $\quad 5$

The free radical theory of aging 6

$\begin{array}{ll}\text { The gycosylation theory of aging } & 9\end{array}$

Types of collagen crosslinks 11

The Maillard reaction theory of aging 13

Hybrid or synergistic theory of aging 13

$\begin{array}{ll}\text { Role of glycosylation in aging process } & 14\end{array}$

$\begin{array}{ll}\text { Pentosidine } & 16\end{array}$

$\begin{array}{ll}\text { Location of glycation crosslinks } & 19\end{array}$

Role of collagen in crosslinking studies $\quad 20$

Diabetes-mediated structural changes in collagen 20

Role of lysyl oxidase in collagen crosslinking 22

Role of Maillard reaction in collagen crosslinking 23 
Role of glycoxidation in collagen crosslinking $\quad 24$

Role of hydrogen peroxide in collagen crosslinking 25

Prevention of glycoxidation derived tissue damage 26

Natural protection 26

Physiological intervention $\quad 28$

Diet restriction 28

Effect of diet restriction on gycosylation $\quad 31$

Role of uric acid in birds $\quad 32$

Pharmacological intervention $\quad 33$

Aminoguanidine 34

Poultry as an animal model for the study of aging 37

CHAPTER 3

Results, discussion and materials and methods 38

Study I

Protein glycosylation and advanced glycosylation endproducts

(AGEs) accumulation: an avian solution? 39

Abstract 39

Introduction 39

Materials and methods $\quad 41$

Results 43

Discussion 44

Acknowledgments $\quad 48$

References 49

Study II

Relationship between mechanical properties and pentosidine in tendon: effect of age, diet restriction and aminoguanidine 62

Abstract

Introduction 
Materials and methods $\quad 64$

$\begin{array}{ll}\text { Results } & 67\end{array}$

Discussion $\quad 68$

Acknowledgments $\quad 71$

$\begin{array}{ll}\text { References } & 72\end{array}$

Study III

Age-related changes in meat tenderness and tissue pentosidine: effect of diet restriction and aminoguanidine $\quad 85$

$\begin{array}{ll}\text { Abstract } & 85\end{array}$

Introduction 86

$\begin{array}{ll}\text { Materials and methods } & 87\end{array}$

$\begin{array}{ll}\text { Results and discussion } & 90\end{array}$

Acknowledgments 93

$\begin{array}{ll}\text { References } & 94\end{array}$

Study IV

Effect of dietary aminoguanidine on tissue pentosidine and reproductive performance 106

$\begin{array}{ll}\text { Abstract } & 106\end{array}$

Introduction 107

Materials and methods 108

Results and discussion $\quad 111$

Acknowledgments 113

References 114

CHAPTER 4

$\begin{array}{ll}\text { Summary } & 126\end{array}$

$\begin{array}{ll}\text { BIBLIOGRAPHY } & 129\end{array}$ 


\section{LIST OF TABLES AND FIGURES}

Study I

Figure 1: $\quad$ Effect of age, diet restriction and aminoguanidine on body weight 56

Figure 2: $\quad$ Effect of age, diet restriction and aminoguanidine on plasma glucose

Figure 3: $\quad$ Effect of age, diet restriction and aminoguanidine on skin pentosidine

Figure 4: $\quad$ Effect of age, diet restriction and aminoguanidine on plasma uric acid

Figure 5: $\quad$ Effect of age, diet restriction and aminoguanidine on oxidative stress

Figure 6: Correlation between age and pentosidine accumulation

Study II

Figure 1: $\quad$ Effect of age and diet restriction on TBS, TBT and tendon pentosidine

Figure 2: $\quad$ Effect of age and aminoguanidine on TBT, TBS and tendon pentosidine

Figure 3: $\quad$ Effect of age, diet restriction and aminoguanidine on TBT, TBS and tendon pentosidine

Study III

Figure 1: $\quad$ Correlations between shear force in pectoralis major muscle and pentosidine

Figure 2: $\quad$ Effect of age, diet restriction and aminoguanidine on SF

Figure 3: $\quad$ Effect of age, diet restriction and aminoguanidine on breast weight 104

Figure 4: $\quad$ Effect of age, diet restriction and aminoguanidine on cook time 105 Study IV

Figure 1: $\quad$ Skin pentosidine in control and AG-supplemented groups 124

Figure 2: $\quad$ Hen day egg production in control and AG-supplemented groups 125

Table 1: $\quad$ Egg production and egg defects 121 
Table 2: $\quad$ Eggshell thickness, egg weight, and specific gravity 


\section{LIST OF ABBREVIATIONS}

\begin{tabular}{|c|c|}
\hline $\mathrm{ACP}$ & Aldol condensation product \\
\hline $\mathrm{AG}$ & Aminoguanidine \\
\hline AGE-R & Advanced glycosylation endproducts receptor \\
\hline AGEs & Advanced glycosylation endproducts \\
\hline $\mathrm{AH}$ & Aldol histidine \\
\hline AL & Ad libitum \\
\hline Br. W & Breast weight \\
\hline BW & Body weight \\
\hline $\mathrm{CB}$ & Cynomide bromide peptide \\
\hline CML & $\mathrm{N}^{\epsilon}$-(Carboxymethyl)-lysine \\
\hline CPM & Count per minute \\
\hline $\mathrm{CT}$ & Cook time \\
\hline CY & Cook yield \\
\hline DHNL & Dihydroxynorleucine \\
\hline DHLNL & Dihydroxylysinonorleucine \\
\hline DR & Diet restriction \\
\hline FFI & 2 Furoyl-4(5)-2(furanyl)-1 H-imidazole \\
\hline Gluc-lys & Glucitolyllysine \\
\hline Gluc-hyl & Glucitolylhydroxylysine \\
\hline HD & Hen day \\
\hline HHMD & Histidinohydroxymerodesmosine \\
\hline HNL & Hydroxynorleucine \\
\hline HLNL & Hydroxylysinonorleucine \\
\hline $\mathrm{LOX}$ & Lysyl oxidase \\
\hline LNL & Lysinonorleucine \\
\hline LP & Lsyl pyridinium \\
\hline
\end{tabular}




$\begin{array}{ll}\text { MDA } & \text { Malanodialdehyde } \\ \text { NFC-1 } & \text { Non fluorescent crosslinks } \\ \text { NADH } & \text { Nicotinamide adenine dinucleotide (reduced) } \\ \text { NO } & \text { Nitric oxide } \\ \text { OHP } & \text { Hydroxypyridinium } \\ \text { P } & \text { Pentosidine } \\ \text { PA } & \text { Pyridinium analogue } \\ \mathrm{P}_{\mathrm{s}} & \text { Skin pentosidine } \\ \mathrm{P}_{\mathrm{t}} & \text { Tendon pentosidine } \\ \text { SF } & \text { Shear force } \\ \text { TBS } & \text { Tendon breaking strength } \\ \text { TBT } & \text { Tendon breaking time }\end{array}$

$x i i$ 


\section{Chapter 1}

\section{INTRODUCTION}

A common feature of the life cycle of virtually all multicellular organisms is the progressive decline in the efficiency of various physiological processes (Sohal and Weindruch, 1996). A variety of strategies and models have been used to understand the nature of the mechanisms underlying the phenomenon of senescence. One hypothesis for aging postulates that the senescence-associated loss of functional capacity is due to the accumulation of molecular oxidative damage (Sohal and Orr, 1995). Nonenzymatic glycosylation or glycation of tissue protein, and subsequent formation of advanced glycosylation endproducts (AGEs) has become of increasing interest in gerontological research for the last two decades, because these processes have been implicated as mediators of the aging process (Cerami, 1985; Monnier, 1989). The glycation theory of aging suggests that modification of proteins by glucose and associated browning or Maillard reaction leads to the gradual crosslinking, polymerization and characteristic development of brown color and fluorescence (Cerami, 1985; Monnier, 1989). These crosslinks are postulated to lead to the deterioration in the structure and function of tissue protein in a manner similar to the mechanism by which the pathophysiology of diabetes generates tissue specific complications (Monnier, 1989; Bucala and Cerami, 1992; Vlassara et al., 1994). One such crosslink, pentosidine, has been isolated and characterized in diabetic subjects and is an imidazole $[4,5 b]$ pyridinium molecule comprising a lysine and an arginine residue crosslinked by a pentose (Sell and Monnier, 1989). Pentosidine is a glycoxidation product, as both glycation and oxidation reactions are involved in its biosynthesis (Baynes, 1991). In mammals, pentosidine has been found to increase in skin, tendons and other tissues over the life span of the individual, and hence can be used as a sensitive biomarker for aging studies (Grandhee and Monnier, 1991; Dyer et al., 1991 b; Cefalu et al., 1995). A comparable increase in biomechanical strength of collagens has been found in vitro after incubation of rat tail tendons 
with glucose (Andreassen and Oxlund, 1985; Kent et al.,1985; Andreassen et al., 1988; Menzel and Reihsner, 1991) and in vivo in animals with experimental diabetes (Galeski et al., 1977; Andreassen et al., 1981). In another study, Verzar (1963) demonstrated that the resistance to thermal denaturation of rat tail tendons, measured as tendon breaking strength (TBT), increased with advancing age. Recent theories have suggested that nonenzymatic glycation reactions play a key role in the phenomenology of aging, either alone or in combination with other processes, such as free radical generation (Masoro et al., 1992; Kristal and Yu, 1992). Such stochastic processes may, in turn, exert a regulatory effect on gene expression during aging (Sohal and Allen, 1990).

Meat of spent fowls becomes objectionably tough with increasing age, and this toughness limits its use in whole muscle foods resulting in considerable economic loss to the poultry industry (Nakamura et al., 1975; Shimokomaki et al., 1972). For this reason, meat has been traditionally used in less-profitable, comminuted or retorted products where the small particle size or thermal processing is used to reduce toughness (Nurmahmudi and Sams, 1997). This problem is of particular importance to the poultry industry which produces spent fowl as a byproduct of table and hatching egg production. Approximately 130,000,000 spent fowl are disposed of in the USA annually (Vandepopuliere and Lyons, 1996); thus, the potential economic benefit to be gained by tenderizing this meat is tremendous. Presently, there is no economically feasible way to increase meat tenderness by decreasing collagen crosslinking in spent fowl meat (Kondaiah and Panda, 1992; Woods et al., 1997).

Advancing age also adversely affects the reproductive success of many species of animals including poultry. A very common observation in broiler breeder hens is the rapid, age-related decline in reproductive performance once the genetic potential for maximum production of eggs has been reached. Furthermore, eggs that are laid during the later period of the reproductive life span are less likely to be fertilized and, if fertilized, are less likely to hatch (Lerner et al., 1993). The factors that influence these age-related decreases in reproductive success are poorly understood. However, there is evidence in male humans that adult onset diabetes accelerates the 
age-related decline in reproductive function and fertility (Kaiser and Korenman, 1988).

Hypothesized that the age-related decline in production and meat quality may be, in part, due to the nonenzymatic glycosylation process, because naturally plasma glucose concentrations in birds are 2-6 times higher than mammals (Holmes and Austad, 1995; Beuchat and Chong, 1996; Iqbal et al., 1998 a). In addition birds also have metabolic rate 2-2.5 times as high as those of mammals of similar body sizes ( Lindstedt and Calde, 1976; Austad and Fischer, 1991) which presumably expose them to a higher rate of free oxygen radical production and, consequently, accelerated tissue damage (Harman, 1956; Del Maestro, 1980); 2) comparatively elevated body temperature (about $3^{\circ} \mathrm{C}$ higher than mammals) (Holmes and Austad, 1995). Both of these latter factors should accelerate the formation of advanced Maillard products. Hence, birds can provide a novel model for ascertaining the role of tissue glycosylation in the age-related functional decline of tissues.

The principal objective of these studies was to understand the role of nonenzymatic glycosylation in birds and to find ways to delay this process so as to improve the productive performance of livestock. Previous studies have shown that diet restriction (DR) is one of the most commonly used measures. It has been shown to increase the mean and maximum life span, decrease age-associated physiological changes, and delay or prevent many of the age-associated diseases (Weindruch and Salford, 1988; Synder, 1989). For example, the reproductive life span of ad libitum-fed broiler breeder is both shorter and less productive than their DR counterpart; DR is the only established means of delaying the decline in their productive performance. The ability of DR to modulate reproductive aging and slow or prevent the onset of a number of major age-associated diseases, makes DR a tremendously powerful and versatile tool (Yu, 1994b). DR also is used successfully in the poultry industry to achieve the desired body weight without affecting reproductive performance (Fontana et al., 1992).

A nucleophilic hydrazine compound, aminoguanidine (AG), that acts as a crosslinking inhibitor and is the most extensively used pharmacological compound (Khatan et al., 1988; 
Odetti et al., 1990; Oxlund and Andreassen, 1992; Wu, 1995). In vivo, AG has been shown to lessen the formation of collagen-linked fluorescence associated with the production of advanced glycosylation endproducts (AGEs). AG has been postulated to block pathological tissue alterations via inhibition of deleterious long-term changes to proteins associated with hyperglycemia (Edelstein and Brwonlee, $1992 \mathrm{ab}$ ). AG has been referred to as a virtually nontoxic nucleophilic hydrazine $\left(\mathrm{LD}_{50}=1800 \mathrm{mg} / \mathrm{kg}\right.$ in rodents) (Ou and Wolff, 1993). AG has been successfully used in poultry to retard the fluorescence in skeletal muscle associated with aging collagen (Klandorf et al., 1996).

Objectives:

The objectives of these studies were to:

1. isolate and quantify the glycoxidation product, pentosidine, from the skin $\left(\mathrm{P}_{\mathrm{s}}\right)$ and tendon $\left(\mathrm{P}_{\mathrm{t}}\right)$ of broiler breeder hens.

2. determine if pentosidine accumulates in the tissues of the hens with advancing age.

3. determine if dietary modulation and supplementation of AG can:

a. retard the rate of accumulation of $\mathrm{P}_{\mathrm{s}}$ and $\mathrm{P}_{\mathrm{t}}$ over the period of time.

b. affect the mechanical properties of tendon.

c. prevent the age-related increase in meat toughness.

d. improve the production performance of broiler breeder hens. 


\section{Chapter 2}

\section{REVIEW OF LITERATURE}

\section{Aging}

Aging is an inevitable biological process that affects living organisms. In general, aging is characteristically described as a time-dependent functional decline, leading to the cell's incapacity to withstand external and internal challenges (Yu, 1996). The causal factors that underlie the time-dependent, deleterious processes of aging have not yet been well defined, and no single adequate molecular explanation for aging is currently available. The consensus among researchers views the concept of biological aging as an organism's failure to maintain homeostasis (Holliday, 1992).

\section{Bases of Aging Hypotheses}

Through the decades, the extent and complexity of aging process has been reflected by a plethora of hypotheses which have inundated the field of aging. According to a recent review, there are more than 300 theories on aging (Medvedev, 1990). An in-depth treatment of these theories is beyond the scope of this review. Most aging theories fall into two broad categories based on putative casual forces: (1) intrinsic factors referring to genomic components and genetic

processes; and (2) extrinsic factors, encompassing various external environmental influences on the organism.

\section{Intrinsic Factors of Aging}

The long-held depiction of aging as a programmed force (Holliday, 1992) is based on observations of various species with finite life spans. The fixed life span of a given species was believed to be predetermined by their genetic makeup, suggesting that the course of 
senescence is actively programmed in much the same manner as the development and maturity phases of the life cycle. The fact that strong genetic influences underlie the course of the aging process is undeniable, as seen in life span differences among species and in cases of accelerated human aging, such as Werner's syndrome. Yet, at present, no compelling reason supports the view that aging is actively programmed by genetic mechanisms (Kowland and Kirkwood, 1994). Crucial unanswered questions then are, to what extent do intrinsic genetic forces play in setting the course of aging?. And, how do these forces interact with environmental factors (i.e., extrinsic forces) to influence the rate of aging?. One emerging clue elucidated from recent molecular studies indicates that intrinsic genomic factors can be readily influenced by environmental factors, such as oxidative stress and DR (Van Remmen et al., 1995; Yu, 1995). A better understanding of the nature of these interactions should provide further insights for many unresolved questions about the causality and process of biological aging.

\section{Extrinsic Factors of Aging}

Most popular theories today postulate a role for extrinsic factors in the aging

process. These theories have centered around the notion that age-related deterioration is primarily related to structural and functional modifications of cellular constituents. Among them are four related hypotheses that implicate free radicals, glycosylation reactions, and /or Maillard reactions as causative factors in the aging process.

The Free Radical Theory of Aging. Investigations over the last two decades have focused a great deal on identifying the extrinsic factors of aging (Eichhorn, 1979). Among them, two of the most likely factors are diet (Nolen, 1972; Holehan, 1986) and free radicals (Orr and Sies 1985; Saul et al., 1987; Porta, 1988; Kehrer and Smith, 1994; Sohal, 1994). These extrinsic factors are particularly significant, because they have characteristic ways of influencing the aging process (Porta, 1988; Farmer and Sohal, 1989; Mote et al, 1991; Kehrer and Smith, 1994; Pahlavani et al., 1994). First, both are inseparable components of an organism's normal metabolism: diet for energy and generation of free radicals during an oxygen metabolism. Second, interaction between these two factors results in deleterious effects directly and indirectly 
on cellular structure and functions. Third, and perhaps more important, both have the capability to interact with intrinsic genetic components which can modify the ultimate course of aging (Farmer and Sohal, 1989; Esterbauer and Schaur, 1990; Mote et al, 1991; Dean et al.,1992; Chaudhry et al., 1994; Pahlavani et al., 1994). Thus, in contrast with programmed aging theories, aging hypotheses based on extrinsic factors recognize the organism's genotypic susceptibility as well as its ability to adjust to a changing environment in the face of external forces (Corpas et al., 1993). Evidence supports the view that abnormal cellular constituents such as DNA, (Cathcrat et al., 1984; Vaca et al., 1988; Brandy and Davison, 1990; Chou et al., 1991; Chung et al., 1992) proteins, (Oimomi et al., 1986; Stadtman, 1988; Baynes and Monnier, 1989; Masoro et al., 1989; Sohal et al., 1993), carbohydrates (Kristal and Yu, 1992; Cadenas et al., 1994; Brownlee, 1995; Wolff, 1995), and lipids are oxidatively damaged by free radicals (Greshman et al., 1954; Yagi, 1991; Yu et al., 1992; Laganiere and Yu, 1993; Chen et al., 1995). Data on cellular damage imply that oxidative modifications of genetic components by these extrinsic factors are consistent with what has been observed in aging and disease processes.

The concept of free radical involvement in aging and disease was proposed by Greshman et al. (1954) and Harman, (1956). These investigators envisioned free radical species causing irrecoverable functional deterioration associated with aging. Gerschaman provided clues to the possible cause of cellular damage from X-ray irradiation, oxygen poisoning, and radiation. In formulating the free radical theory of aging, Harman deemed oxygen free radicals as the primary underlying cause of age-related deterioration and disease leading to death. This aging theory is meritorious for having provided modern gerontologists with both a conceptual base and the experimental opportunities necessary to explain the biological basis of senescence and disease.

More knowledge gained in the field of free radical biochemistry over the last decade necessitated an expansion and modification of the original free radical theory of aging ( $\mathrm{Yu}$ and Yang, 1996). Free radicals are defined chemically as atoms or molecules that are highly reactive due to an unpaired electron in an outer orbit (Del Maestro,1980). Because of the reactivity due to the unstable electronic configuration, free radical reactions proceed randomly by chain 
reaction (Oldham et al., 1988). Although not itself a free radical, the biologically important molecule $\mathrm{H}_{2} \mathrm{O}_{2}$ can undergo the trace metal $(\mathrm{Fe} / \mathrm{Cu})$ catalyzed Haber-Weiss (Aust, 1985) and Fenton reactions (Halliwell and Gutterridge, 1984), leading to the generation of highly reactive hydroxyl radicals.

Most biological substances are targets of free radical attack, and the ubiquitous, nonselective, and random nature of free radical reactions make the free radical theory of aging among the most plausible mechanisms to explain both aging processes (Harman, 1956; Porta, 1988) and major age-related diseases such as cancer (Sun, 1990) and atherosclerosis (Del Maestro, 1980). The most important factor implicating free radicals in the aging process is the fact that under normal physiological conditions, the majority of these free radicals are generated endogenously from inspired $\mathrm{O}_{2}$ molecules as by-products of the mitochondrial electron transport reactions involved in energy production. This inherent nature and absolute oxygen requirement for biological systems make it extremely difficult to eliminate the possibility that free radicals do not play causal roles in aging processes.

Although major cellular sources of free radicals are mitochondria, microsomes, and peroxisomes, sources of reactive species are widespread throughout the body, (Freeman, 1984; Boje and Arora, 1992; Moncada et al., 1992; Masters, 1994), for example, intracellular enzymes reactions formed by oxidases, dehydrogenases and oxygenases and extra cellular sources of oxidant species form glycation and Maillard reactions (Kristal and Yu, 1992). Nitric oxide synthase has been shown to be another significant oxidant source (Boje and Arora, 1992; Moncada et al., 1992 Masters, 1994). Emerging evidence shows the generation of reactive aldehyde compounds from the lipid peroxidation process (Esterbauer and Schaur, 1990; Chen et al., 1995; Kristal et al., 1996). An estimated 1-2\% of mitochondrial oxygen consumption is converted, by simple electron transfer, to the formation of the superoxide radical (Bandy and Davison, 1990). Mitochondrial production of superoxide has been estimated to be as high as $10^{7}$ superoxide radicals/day (Nohl and Hegner, 1990; Guarnieri et al., 1993). It has been estimated that a single cell in a living organism may be exposed to $10^{10}$ molecules of superoxide radical 
$\left(\mathrm{O}^{\bullet}\right)$ per day (Ames et al., 1993) representing $1.75 \mathrm{~kg}$ of $\mathrm{O}^{\bullet}$ produced in a $70 \mathrm{~kg}$ human over a one year period (Frei, 1994). This would roughly translate to a range of 2 to $5 \mathrm{gm}$ of $\mathrm{O}^{\bullet}$ being produced in a broiler from 0 to 49 days of age (Bottje et al., 1995).

Consequences of Free Radical Attack. Although free radical prodution per se does not appear uniformly increased with age, the damage induced, e.g., lipid peroxidation, is detected very widely (Yu, 1994). Lipid peroxidation can have numerous detrimental effects on cell function (Horton and Fairhurst, 1987) and membrane fluidity (Yu, 1994). For example, there is evidence that $\mathrm{Na}^{+} / \mathrm{K}^{+}$ATPase activity is disrupted by lipid peroxidation (Thomas and Reed, 1990), resulting in an inability of the cell to maintain the electrical gradient required for cell viability. An increase in cytosolic $\mathrm{Ca}^{2+}$ following oxidative induced damage to membrane $\mathrm{Ca}^{2+}$ ATPase causes the loss of cytologic $\mathrm{Ca}^{2+}$ regulation and has been suggested as a key step leading to cell death (Thomas and Reed, 1988). Free radicals can also oxidize proteins and DNA within the cell, while extracelluar release of reactive oxygen species (ROS) can damage neighboring cells. Another group of toxic compounds formed during lipid peroxidation are the 4hydroxyalkenals which react with sulfhydryl groups of proteins, and along with melanodialdehyde (MDA), can convey the toxic effects of lipid peroxidation throughout the cell (Bottje et al., 1995).

The Glycosylation Theory of Aging. The implication of glycation or glycosylation reactions in the aging process (Monnier and Cerami, 1981;Cerami, 1985) can be considered as an expanded version of the collagen crosslinking theory of aging, and is based on the crosslinking of numerous proteins by modified glucose residues. Indeed, at the heart of the glycation theory (Cerami, 1985) is the hypothesis that these crosslinks impair both cell and tissue function, which thus leads to age-associated tissue deterioration. The glycosylation theory came in the aging field

somewhat circuitously through observations concerning the increased development of specific pathologies in diabetic patients and their similarities to those in the aged population. This 
association lead to the linkage between the glycation process and Maillard reactions as possible mechanisms underlying the accelerated aging of diabetics.

\section{Types of Collagen Crosslinks}

Collagen crosslinking is a complex post-translational modification that occurs extracellularly (Yamauchi and Mechanic, 1987; Fujimoto, 1988; Reiser et al., 1992). Collagen crosslinks appear to arise from one of two general biosynthetic pathways (Reiser, 1991; Yamauchi and Mechanic, 1987). Crosslinks may be classified into two major groups: those mediated by the enzyme lysyl oxidase (Siegel, 1979; Reiser et al., 1992) and those derived from the nonenzymatic addition of glucose adducts (Baynes and Monnier, 1989; Reiser, 1991).

Enzymatic Collagen Crosslinks. A crosslink pathway begins with the lysyl oxidasemediated oxidative deamination of certain lysine and hydroxylysine residues. The resultant aldehyde moieties may undergo further reactions with each other or with lysine, hydroxylysine and histidine residues to form a series of di-, tri- and tetrafunctional crosslinks in collagen (Robins, 1982).

In some tissues and species, aging is associated with an increase in non-reducible mature crosslinks, the reaction products of difunctional enzymatic crosslinks and unmodified residues (Fujimoto, 1982; Yamauchi et al., 1987). However, in many tissues the generation of mature crosslinks tends to plateau with age, rather than increase in a linear fashion, and thus are unlikely to account for the progressive physiochemical changes associated with age. In contrast, there is considerable evidence that nonenzymatically mediated collagen crosslinks derived from glucose adducts increase with age in virtually all tissues studied. The accumulation of such compounds, so called advanced glycation endproducts (AGEs), is associated with alterations in mechanical properties, solubility, ligand binding and conformation of collagen (Reiser, 1991). Recent theories have suggested that nonenzymatic glycation reactions play a key role in the phenomenology of aging, either alone or in combination with other processes, such as free 
radical generation (Masoro et al., 1989; Kristal and Yu, 1992). Such stochastic processes may in turn, exert a regulatory effect on gene expression during aging (Sohal and Allen, 1990).

Glucose Derived Nonenzymatic Collagen Crosslinks. The second pathway begins with the nonenzymatic glycation of lysine and hydroxylysine residues. These early glycation products, glucitolyllysine and glucitolylhydroxylysine, are believed to undergo a series of reactions to form complex fluorophores and chromophores, collectively referred to as advanced glycation endproducts (AGEs) (Reiser, 1991). There is now considerable evidence that collagen crosslinks arise from nonenzymatic glycation accumulate in virtually every tissue of diabetic subjects (Reiser, 1991), and that such abnormalities are associated with changes in the physiochemical properties of collagen and ultimately with long-term clinical complications.

The nonenzymatic reaction between glucose and proteins is collectively known as Maillard reaction. On the basis of the recent in vitro and in vivo studies, it is proposed that this nonenzymatic gycosylation of certain proteins in body triggers a series of chemical reaction that culminate in the formation, and eventual accumulation of irreversible crosslinks. It begins with the formation of schiff bases when an aldehyde group (CHO) of glucose and an amino group $\left(\mathrm{NH}_{2}\right)$ of a protein are attracted to each other. This combination is unstable and quickly rearranges itself into a stabler, but still reversible, substance known as an Amadori product. If a protein persists in the body for months or years, some of its Amadori products slowly dehydrate and rearrange themselves yet again- into new glucose-derived structures. These can combine with various kinds of molecules to form irreversible structures named AGEs. Most AGEs are yellowish brown and fluorescent and have specific spectrographic properties. More important for the body, many are also able to crosslink with adjacent proteins. The precise chemical structure of advanced glycosylation end products and of most AGE-derive crosslink is still not known. Nevertheless, some evidence suggests that AGEs are often created by the binding of an Amadori product to glucose or another sugar. Such end products would form bridges to other proteins by binding to available amino groups. In some instances two Amadori products may instead merge, creating an AGE that is also a crosslink. The first such crosslink isolated is 2- 
furoyl-4(5)-(2-furanyl)-1H-imidazole, or FFI, mixture of amino acid lysine, albumin and glucose (Cerami et al., (1987), but since been shown to be an artifact of the isolation procedure.

The Maillard Reaction Theory of Aging. Monnier (1989) and Monnier et al., (1991) expanded the glycation theory of Aging to include all Maillard reactions, the class of chemical reactions to which glycation reactions belong. The Maillard reaction represents a series of complex processes that include initiation, propagation, and termination processes similar to those seen in free radical reaction (Monnier et al., 1991). Maillard reactions may be initiated by any compound containing an $\propto$ - hydroxy aldo or keto group reacting with the $\epsilon$-amino group of an amino acid. A typical reaction producing glycated proteins is the formation of Amadori products via a Schiff base resulting from a condensation reaction between the amino groups of proteins and aldo or keto moieties of reducing sugars (Wolff et al., 1987). The Amadori product can degrade further to various ketoaldeydes, each of which contains reactive carbonyl groups (Monnier, 1990). In some cases, these reactions require transition metals such as $\mathrm{Fe}$ and $\mathrm{Cu}$, as do some free radical reactions (Mullarkey et al., 1990). The secondary substances are often more reactive than the parent compound which can then generate from macromolecular complexes. The propagation processes, including fragmentation and crosslinking, eventually lead to advanced Maillard products (Monnier et al., 1991) termed as AGEs (Monnier and Cerami, 1981).

Synergistic Theory. This recent theory proposed by Kristal and Yu, (1992) is comprised of the free radical, glycation, and Maillard reaction theories of aging: the essence of the proposal is based on the consideration that each of these three processes share common chemical reactions that involve free radicals. This new theory of aging is based on the prediction that agerelated deterioration is produced by the sum of the damage induced by free radicals, by glycation and other Maillard reactions, and by their interplay. By combining the free radical, glycation, and Maillard theories of aging, (Yu, 1993) this theory postulates that these separate processes, which differ significantly in their site of generation and their primary targets, might interact to cause age-associated deterioration. The synergistic interactions suggested by this theory offers 
a stronger scientific basis for the direct involvement of a Maillard reactions, including glycation, in the aging process, rather than their indirect involvement coupled to their association with agerelated diseases.

\section{Roles of Glycation and Oxidation in Aging Process}

The extent of glycation or glycosylation of tissue proteins depends, in part, glucose concentrations and is increased in proportion to the degree of hyperglycemia in diabetes. Although glycation is relatively constant with age (Dyer et al., 1992), other products of reactions with sugars and proteins accumulate in long-lived tissue protein (Dunn et al., 1989; Dunn et al., 1991). These compounds, such $\mathrm{N}^{\epsilon}$-(carboxymethyl)lysine (CML) and pentosidine (P), as well as a characteristic Maillard-type fluorescence are generated during the Maillard or browning stages of the glucose-protein reaction. CML and P are termed as glycoxidation products because both glycation and oxidation reactions are required for their formation from reducing sugars (Dyer et al., 1991; Baynes, 1991). Strong correlations have been noted between CML, P, and fluorescence in the control and diabetic populations, providing evidence of age-dependent chemical modification of skin collagen via the Maillard reaction and acceleration of this process in diabetes. (Dyer et al., 11992).

Oxygen may have a special role as a fixative of chemical damage to proteins by the Maillard reaction in vivo, as glycoxidation products are the only products of Maillard reaction known to accumulate in tissue proteins (Dyer et al., 1991; Baynes, 1991). The increase in the rate of their accumulation in collagen in diabetes is consistent with the description of diabetes as a disease of accelerated chemical aging of tissue proteins. The accumulation of CML and P is also consistent with the free radical theory of aging which considers aging to be the result of cumulative oxidative damage to biomolecules. Despite the difference between the structures of CML and $\mathrm{P}$, the two compounds accumulate in concert with normal aging and their rates of accumulation are comparably accelerated in diabetes. For this reason, they provide a consistent index of protein exposure to oxidative stress AGEs (Reiser, 1991). Glycoxidation products appear to be particularly sensitive indicators of oxidative stress, perhaps because of ease of 
oxidation of reducing sugars and their adducts to protein. Although CML and P probably account only for a fraction of the carbohydrate adducts and crosslinks formed nonenzymatically in protein, both appear to be useful as biomarkers of the extent to glycative and oxidative damage of protein via the Maillard reaction (Dyer et al., 1991; Baynes, 1991).

Pentosidine. This compound is the most widely described Maillard structure in the context of extracellular matrix glycation. First isolated by Sell and Monnier (1989a) its structure was described soon afterwards as an imidazopyridinium compound (Sell and Monnier, 1989b), comprised of single lysine and arginine moieties crosslinked by a pentose. FAB-high resolution mass spectrometry showed a $\mathrm{m} / \mathrm{z}$ of 379,2069 compatible with the empirical formula $\mathrm{C}_{12} \mathrm{H}_{27} \mathrm{~N}_{6} \mathrm{O}_{4}$. Pentosidine could be synthesized in vitro by reacting L-lysine with L-arginine and D-ribose of collagen or with D-ribose or its isomers i.e., the subsequent crosslink was given the name of pentosidine. However, preliminary data suggested that plasma ribose levels are very low in patients with diabetes. It has now been identified as the endproduct of the Maillard reaction elicited by hexoses, pentoses, ascorbate and a variety of Amadori compounds in vitro (Sell and Monnier, 1989a, 1989b; Dyer et al., 1991; Grandhee and Monnier, 1991). The proposed mechanism of pentosidine formation from D-ribose, D-glucose, D-fructose, and Lascorbate the common intermediate I. However, the major in vivo carbohydrate source leading to pentosidine formation is not known. What clear is that oxidation reactions are required at some stage in the reaction as the formation of pentosidine is inhibited in the absence of oxygen, a property that prompted Baynes to coin the phrase 'glycoxidation product' to describe pentosidine and other glycation compounds similarly affected by oxidation availability (Baynes, 1991).

Detection of pentosidine in the tissues of animals has been greatly facilitated by its acid stability, and more significantly by its fluorescent properties. Consequently, it has now been, identified in numerous collagenous and non-collagenous tissues and shown to exhibit a linear increase with age in normal tissue (shrew, monkey, dog, pig, cow, rat, human) (Sell and Monnier 
1989; Sell et al., 1996) whereas in patients with diabetes, there is an accelerated rate of increase. However, its significance as a major crosslinking component has been largely discounted. Dyer et al., (1991) initially presented evidence that pentosidine accounts for $<1 \%$ of the total crosslinks formed during the in vitro browning reaction of proteins with glucose. This figure is based on a yield of only one pentosidine per 100 collagen molecules, representing $1 \%$ of the assumed potential of 1 crosslink per collagen molecule. It is now regarded mainly as a biomarker in the

assessment of cumulative damage to proteins by nonenzymatic reactions of the Maillard reaction. In view of the low yields of fluorescent crosslinks observed during in vitro and in vivo investigations, Bailey et al., (1995) turned their attention to a detailed analysis of non-fluorescent components associated with highly crosslinked collagen. Using a modified technique developed for the detection of lysyl oxidase mediated crosslinks (Sims and Bailey, 1992) they were able to identify a non-fluorescent component, NFC-1, the concentration of which could be correlated to a change in phyiochemical properties associated and is detected in HPLC analysis as a major ninhydrin positive peak eluting after lysine. NFC-1 is generated following short incubations of collagen with ribose (3 days) or glucose albeit after more extended incubation (3 month). Data from Paul and Bailey (1996) indicated that NFC-1 is composed of lysine and arginine moieties. It can be formed by the reaction of $\mathrm{N}$-acetyl-lysine, $\mathrm{N}$-acetyl-arginine with either ribose or glyoxal, a retro-aldol cleavage product of the Maillard reaction. The concentrations of NFC-1 are about 50 times greater than that of pentosidine in aorta (Sims et al., 1996)

Location of Glycation Crosslinks. The location of the potential crosslinks in the collagen fiber, has not been elucidated. The decreased susceptibility of collagen to enzymes and the increased thermal denaturation temperature following glycation is consistent with inter helical crosslinking, which may involve lysines at random location along the length of the molecule, or preferentially glycated lysine. It is these lysines that might also be the site of the crosslinks. Studies on the location of the initial hexosyllysine in young and old rat tendon collagen by Reiser et al., (1992), rather surprisingly indicated a preferential glycation of particular lysines in the cyanogen bromide peptide (CB-3) in the middle of the collagen molecule, since one might expect 
the lysines in the rod-line molecule to be reasonably equivalent throughout the length of the molecule. Wess et al., (1993) provided evidence from neutron scattering for increased glycation in the gap region of the fibre. The gap region of the collagen fiber is formed as a result of the end-overlap quarter stagger alignment of the molecules in the fiber. The end-overlap is a region of more tightly bound molecules whilst the gap or hollow region contains less and loosely bound molecules. This latter region might be accessible to glucose and the formation of glycation products as shown by Wess and his colleagues.

\section{Collagen in Crosslinking Studies}

In the past 15 years, considerable efforts have been devoted to understanding the nature of postsynthetic collagen modifications associated with diabetes (Monnier et al., 1996). The focus on collagen is warranted for several reasons. First, collagen is present ubiquitously (25 to $30 \%$ of mammalian proteins), and changes in collagen structure occur in several tissues that are critically affected by diabetes, such as joints, arteries, the capillary bed in the retina, and the renal glomerular system. Second, the long half-life of collagen increases its susceptibility to accumulate molecular lesions. Thus, the biochemical nature of these changes in structure, if understood, may provide a window into the pathogenesis of diabetic complications in general. Third, the determination of specific collagen changes in skin may provide a much needed marker to monitor midterm efficacy of therapeutic intervention. (Monnier et al., 1996).

\section{Effect of Glucose on Collagen Structure}

A number of changes occur at the tissue level in diabetes involve collagen. In humans, these include the rapid development of atherosclerosis (Minaker, 1987), thickening of capillary basement membranes (Kilo et al., 1972), increased arterial wall stiffness (Pillsbury et al., 1974), decreased lung elasticity (Schuyler et al.,1976), sclerosis of renal glomeruli (Bilous et al., 1989), stiffening of the heart (Regan et al., 1977), periarticular rigidity (Grgic et al.,1975), and osteoarthritis (Hadley, 1993). Some of these changes also are observed in the diabetic and galactosemic animal, such as capillary basement membrane thickening (Robison et al., 1983; Chandler et al., 1984; Klandorf et al, 1996) and glomerulosclerosis (Velasquez et al., 1990). 
Data from animal studies, especially the diabetic rat, reveal profound abnormalities in the mechanical properties of tail tendons such as tensile behavior, stress strain behavior, and crimp length, as well as a tendency toward a larger fibril diameter (Galeski et al., 1977). Thermal stability is increased in collagen from tendons (Yue et al., 1983) and skin (Yosha et al., 1983) of diabetic rats. Skin collagen solubility to acid and pepsin also is decreased (Ramanurthy et al.,1972), and its strength and stiffness are increased (Andreassen et al., 1981). Similarly, extractability of collagen by acid and pepsin from aortas of diabetic rats is decreased (Brownlee et al., 1986), concomitant with an increase in the tensile strength of the aortic wall is increased (Andreassen and Oxlund, 1987).

At the molecular level, collagen in various tissues from diabetic individuals is less digestible by collagenase and cyanogen bromide (Francis et al., 1974; Hamlin et al., 1975; Kohn and Schnider, 1982; Schnider and Kohn, 1982; Kohn, 1983), and the pepsin releasable fraction of skin collagen contains more high molecular weight material than that from age-matched control subjects (Kohn and Schnider, 1982). X-ray diffraction studies of toe tendons from diabetic individuals reveal alterations in the lateral packing and axial structure of collagen molecules in diabetes (James et al., 1991).

Although most of the changes described above are non-specific parameters of crosslinkng, a number of investigators were able to bring additional evidence for the presence of high molecular crosslinked material in collagen extract using SDS-PAGE (Schnider and Kohn, 1982; Brownlee et al., 1986). Thus, there is strong evidence that intra- and intermolecular crosslinks form at an accelerated rate in the extracellular matrix in diabetes.

\section{Role of Lysyl Oxidase in Age-related Collagen Crosslinking}

Lysyl oxidase (LOX) is the enzyme involved in the formation of the so-called physiological crosslinks that are needed for the stabilization of collagen fibrils. Drugs that inhibit the formation of LOX induce a condition called lathyrism in which collagens are poorly crosslinked with the result that arteries are at increased risk of rupture (Narayanan et al., 1982). 
Thus, the absence of the enzyme may have profound effects on development (Butler et al., 1987). The finding that LOX activity is increased in lungs from diabetic rats (Madia et al., 1979) has intrigued several investigations in this field.

Evidence supporting a role for LOX in collagen crosslinking in diabetes includes the inhibition of acid-soluble crosslinkages by $\beta$-aminopropionitrile (a LOX inhibitor) and Dpenicillamine, which complexes lysine- and hydroxylysine-aldehyde groups (Chang et al., 1980). It has been demonstrated that some of the specific LOX-dependent crosslinks are increased in experimental (Chang et al., 1980) as well as human diabetes (Buckingham and Reiser, 1990).

Whereas LOX crosslinks may well contribute to the increased stability of newly synthesized collagen, such as in granulation tissue and perhaps during sclerosis, a number of observations suggest that these crosslinks are unlikely to completely explain the dramatic stiffening of tissues and crosslinking of collagen in diabetes. First, LOX-mediated crosslinks appear to form in newly synthesized collagen in very specific sites and are progressively replaced by more stable physiological crosslinks (Fujimoto, 1982; Reiser et al., 1992). There is no evidence that diabetes can catalyze LOX crosslink formation in sites other that those involved during physiological maturation of collagen. Second, the tensile strength of aortic wall collagen of diabetic rats is increased, but LOX-mediated crosslinks are unchanged as compared with controls (Andreassen and Oxlund,1987). Because collagen content in several tissues such as skin (Rodnan et al., 1979; Vishwanth et al.,1986), arteries (Rehkter et al., 1993), kidneys (Klein et al.,1975), and heart (Regan et al.,1977) is increased, findings of elevated LOX crosslinks in such tissues in diabetes is best interpreted as a reflection of newly deposited collagen.

\section{Role of The Maillard Reaction in Collagen Crosslinking}

The proposition that the Maillard reaction would play a role in collagen crosslinking was first formulated by Bensusan (1965) but went unnoticed. Similarly, earlier findings that collagen contains glucitolyl-lysine adducts (Robins and Bailey, 1972) did not trigger an investigation into the role of the Maillard reaction in diabetes and aging until nonenzymatic glycosylation was 
proposed as a mechanism for diabetic complications (Cerami et al., 1979). By this time, several studies by Kohn and Schnider (1982) and others demonstrated that diabetes has several features of accelerated aging, both at the tissue level and at the level of collagen itself. Thus, the concept that reducing sugars such as glucose could explain the progressive crosslinking of collagen during normal aging and at an accelerated rate in diabetes (Monnier and Cerami, 1981; Monnier et al., 1984) warranted in-depth studies into the role of glucose as a crosslinking agent of the extracellular matrix. Many studies confirmed the fact that incubation of collagen with glucose or other glycating sugars could reproduce conditions associated with diabetes and normal aging. For example, the increase in thermal rupture time in diabetic and aging rats is duplicated by incubation in glucose (Yue et al., 1983; Andreassen and Oxlund, 1985) or glucose-6-phosphate solutions (Kohn et al., 1984).

The implication of glycated lysine residues or some protein-bound carbonyl intermediates in the formation of crosslinks suggest the role of Amadori products in collagen crosslinking. Several studies have firmly established that collagen glycation is increased during exposure to high glucose in vitro (Fujimoto 1977; Guitton et al., 1981; Rogozinski et al.,1983 Guitton et al., 1984; Perejda et al., 1984; Fujimoto and Horiuchi, 1986; Andreassen et al., 1988; Fu et al., 1994) or in vivo (Kohn and Schnider, 1982; Perejda and Uitto, 1982; Yue et al., 1984; 1985; Davis and Stehbens, 1984; Le pape et al., 1984 Oimomi et al., 1986; Vishwanth et al.,1986; Brennan, 1989; Monnier et al.,1988; Lyons et al.,1991; Miksik and Deyl, 1991; Miksik et al.,1991) and that collagen glycation increases with age in several tissues (Kohn ans Schnider,1982; Ansdreassen and Oxlund 1985; Oimoni et al.,1986; Miksik et al.,1991). Yet, other studies have failed to document anything more than a loose relationship between crosslinking and glycation, either in vitro or in vivo. For example, collagen glycation, although short-term, does not inhibit collagenase digestibility (Lyons and Kennedy, 1985). Increased mechanical stability of tail tendons during glycation in phosphate buffer is prevented when glycation carried out in Tris buffer in spite of equal extent of glycation in both buffers (Andreassen and Oxlund, 1985; Lyons and Kennedy, 1985; Andreassen et al., 1988). Treatment of diabetic rats with aspirin or naproxen, a nonsteroidal anti-inflammatory agent, prevents the 
increase in thermal rupture time without decreasing glycation (Yue et al.,1985; Yue et al.,1984). Similarly, treatment of diabetic rats with vitamin E limits collagen crosslinking without affecting glycemia or glycated hemoglobin levels (Aoki et al.,1992).

Role of Glycoxidation in Collagen Crosslinking. Studies by Baynes and colleagues (Baynes, 1991) demonstrated that proteins that are oxidatively stressed generate a fluorescent product similar to those formed during incubation of proteins with reducing sugars. In subsequent studies, Baynes and colleagues (Fu et al.,1994) showed that covalent crosslinks form concomitantly with increasing glycation, fluorescence, and carboxymethyllysine (CML) and pentosidine levels during incubation with glucose and that anaerobic conditions suppress all modifications except the formation of Amadori products, i.e., glycation itself. Addition of antioxidants and free radical scavengers have a similar effect. Thus, these experiments show that it is possible to uncouple glycoxidation and crosslinking from glycation (Fu et al., 1994). When aminoguanidine (AG), a crosslinking inhibitor, was used in these studies, it also blocked glycoxidation, thereby implicating ketoaldehydes in addition to reactive oxygen species in the crosslinking process. The question, however, is whether the ketoaldehydes originate from oxidation of Amadori products (glycoxidation) or from an earlier step, i.e., glucose autoxidation (Wolff and Dean, 1987) or oxidation of Schiff base intermediates (Namiki and Hayashi, 1975). Based on kinetic analysis, Fu et al., (1994) concluded that Amadori products are not likely to be the major precursors of collagen crosslinks, but that direct autoxidation of glucose, perhaps catalyzed by amino groups, may be as important as glycation. In contrast, Hunt et al. (1993), using a system comprising bovine serum albumin, glucose, and copper, came to the conclusion that oxidation of Amadori products plays a more important role in protein modifications than glucose autoxidation.

To clarify this discrepancy, Glomb and Monnier (1995) followed CML formation as a function of glycoxidation in a glucose/lysine incubation system and used $\mathrm{AG}$ and boric acid as trapping agents for free glyoxal and Amadori products, respectively. Results showed that when the reaction is initiated from glucose, AG traps $50 \%$ of CML. However, CML formation is 
hardly suppressed when it originates from Amadori products. In contrast, boric acid totally suppressed CML formation from Amadori products, but suppressed only $37 \%$ of the glucose/lysine system. These and other experiments (Glomb and Monnier, 1995) suggest that in a glucose/lysine system $~ 50 \%$ of CML formed originates from oxidation of Amadori products and $\sim 40-50 \%$ from a pre-Amadori stage largely independent of glucose autoxidation, the socalled Namiki pathway.

Role of Hydrogen Peroxide in Collagen Crosslinking. The studies from other laboratories (Jiang et al., 1990; Mullarkey et al.,1990), implicate oxygen-reactive species in protein modification during incubation with glucose. To further determine the role of glucose and Amadori products in collagen crosslinking, Elgawish et al. (1997) added $2 \mathrm{mmol} / \mathrm{l}$ glycated $\epsilon$-amino-capric acid to rat tail tendons incubated with 5 and $30 \mathrm{mmol} / \mathrm{l}$ glucose. A dramatic increase in crosslinking as measured by thermal stability was observed. A substantial part of crosslinking could be suppressed with catalase, thereby implicating $\mathrm{H}_{2} \mathrm{O}_{2}$ in the crosslinking process.

To evaluate the role of $\mathrm{H}_{2} \mathrm{O}_{2}$ in collagen crosslinking in vivo, intact tail tendons were implanted into the peritoneal cavity of diabetic rats, which were then treated for 28 days with intraperitoneal injections of catalase or a control protein. Again, significant inhibition of tail tendon crosslinking was observed, which could also be duplicated with antioxidants (Elgawish et al., 1997).

\section{PREVENTION OF GLUCOSE-DERIVED TISSUE DAMAGE}

Knowledge of the mechanisms involved in collagen crosslinkings has led to attempts to alleviate or inhibit the effects of AGEs. An interesting suggestion is that the body possesses a system for removing some of these AGEs and damaged cells from the body. For this reason, the protection against Maillard reactants can be divided into three categories: 1) natural ; 2) physiological and; 3) pharmacological intervention. 
Natural Protection Against Maillard Reactants. Defense against toxic carbonyl compounds derived from sugars or other sources are present and this involves:

Glucose as a Sources of Energy. Bunn and Higgins (1981) demonstrated that glucose as the major carrier of energy in mammalians, precisely because it is the slowest reacting carbohydrate. It is apparent that other sugars such as ribose are 130 times more reactive in the browning reaction than glucose. Thus, the lower the plasma and cellular concentration of these highly reactive sugars, the lesser the biological stresses to an animal system. Furthermore, both plasma and cells have high levels of free low-molecular-weight amines that can compete with glucose, and thus act as physiological inhibitors of glycation.

Protection by Oxidative Fragmentation of Amadori Products. Amadori products can undergo oxidative fragmentation in the presence of $\mathrm{Fe}^{2+}$ and oxygen to form carboxyalkylated lysine residues. Fragmentation of the Amadori product of glucose between $\mathrm{C} 2$ and $\mathrm{C} 3$ gives rise to $\mathrm{N}^{\epsilon}$-carboxyalkylated lysine and erythronic acid, whereas fragmentation between $\mathrm{C} 3$ and $\mathrm{C} 4$ results in the formation of 3-( $\mathrm{N}^{\epsilon}$-lysino)-lactic acid and glyceric acid. The net effect is that the protein has lost a positive charge and gained a negative charge. Furthermore, the modification is irreversible. Thus, the formation of carboxy-alkylated protein may be detrimental to protein function. On the other hand, however, it might prevent irreversible inter- or intramolecular crosslinking and protein trapping during advance Maillard reactions (Brownlee et al., 1983; Brwonlee et al., 1985). This mechanism of protection might be particularly important in the prevention of excessive crosslinking on the extracellular matrix, the hallmark of the aging process.

Protection against Maillard Reactants through Enzymatic Inactivation. Hata et al., (1988) has shown that liver enzymes such as $\alpha$-ketoglutaraldehyde dehydrogenase in the presence of $\mathrm{NADH}$ can use 3-deoxyglucosone as a substrate, thereby suggesting that Maillard reactants could be inactivated enzymatically. Although there is no evidence that Amadori 
products themselves could be cleaved enzymatically, the notion that Maillard intermediates that propagate the reaction could be inactivated enzymatically is important.

Protection by Endogenous Amines That Can Trap Maillard Reactants. The concept that one might be able to interfere in the course of the Maillard reaction comes from the work of Brownlee et al. (1986) who showed that AG can block the formation of protein-linked fluorescence and crosslinking in model systems in vitro as well as in diabetic rat skin collagen. Based on this concept, it is thus logical to consider amines not only for their ability to initiate the Maillard reaction by reacting with sugars, but also for their potential as scavenging agents of the highly reactive Maillard intermediates.

\section{Protection by Cellular Removal and Degradation of Advanced Maillard Molecules.} Vlassara et al., (1984 a; b; 1987; 1988) demonstrated that macrophages have the ability to remove molecules or cells modified by AGEs. From their work evolved the notion that some cells have a specific receptor (AGE-R) that recognizes the modified protein, thus preventing the accumulation of AGE proteins. When myelin was glycated with glucose or glucose 6-phosphate, it was more rapidly taken up by macrophages compared to myelin incubated without sugar. Similar observations were made with AGE-modified albumin.

Further study by Vlassara and Bucala (1996) demonstrated the presence of cell surface receptors that were specific for the recognition and degradation of AGE-modified proteins also have been identified on circulation monocytes, lymphocytes, endothelial and renal mesangial cells, and other cellular systems to partake in both normal tissue remodeling and tissue damage. Interaction of AGE-modified proteins with AGE-R complex serves not only to degrade AGE proteins but also to induce the synthesis and release of cytokines (Vlassara et al., 1988) and growth factors (Kirstein et al., 1990, 1992), suggesting a dual purpose: disposal of senescent AGE-modified molecules and initiation of tissue repair and protein turnover. 
Physiological Intervention. Throughout history, humans have sought a means to a longevity, (Busse, 1973; Comfort, 1979) but few have been successful in their pursuit of successful antiaging strategies (Yu, 1995). Thus, far, DR is the only life-prolonging means accepted by experimentalists. DR has consistently proven to extend both median and maximum life spans of laboratory animals, owing probably to its ability to intervene in both biological aging and life-shortening pathogenesis (Weindruch and Walford, 1988). In addition, evidence from the last two decades of DR research has established the credibility of DR as the most potent modulator of both physiological and pathologic processes. The following sections explain the unique abilities of DR as a research tool to further define the aging process.

Mechanisms of Action of DR. The following are the different hypotheses presented to explain the underlying mechanism of DR. The ability of DR to modulate life span and retard pathogenesis has merited it the gold standard of experimental gerontology in corroborating or disproving several major aging hypotheses. (Masoro, 1988; Weindruch and Walford, 1988).

The Growth and Development Hypothesis. The growth and development hypothesis is based on one of the oldest notions of aging, i.e., that the rate of growth is positively correlated with the rate of aging (i.e., the slower the growth, the longer the life span). The phenotypic consequence of slow growth under various conditions was taken as evidence of lifeprolonging action. The validity of this hypothesis was tested by implementing DR after sexual maturity, or in mid life, in order to avoid the period of slow growth and to not interfere with development (Weindruch and Walford, 1988; Yu, 1994). Amazingly, the results of these studies clearly indicated that the rate of growth has little bearing on a life span; thus, DR provided evidence refuting the growth retardation theory.

The Glucocorticoid Cascade Hypothesis. A more recent hypothesis of aging is the glucocorticoid cascade hypothesis (Sapolsky et al.,1986). The basic tenet of this proposal contends that chronically elevated glucocorticoid levels are the underlying factor of chronic 
stress leading to neuronal death. Several studies support this claim by linking chronic stress to experimentally induced accelerated aging. Other supporting evidence shows that the hippocampus undergo a neural cell reduction with age. If this theory holds true, one could predict that glucocorticoid levels will be higher in ad libitum-fed rats than in DR rat. Studies by Sabtino et al., (1991) examined rats at various ages by taking blood samples several different times during the day to detect diurnal hormone secretions. Surprisinlg; throughout their life, DR rat had significantly higher levels rather than the predicted low levels of the stress hormone. Although further investigation is needed, the results from the DR studies suggests that the major role of glucocorticoid in aging is not as an accelerator of the aging process.

Metabolic Rate Hypothesis. Another theory of aging examined by the DR paradigm is the metabolic rate hypothesis. This theory has been deeply rooted in gerontological literature (Masoro et al., 1982). The original proposal has based on the rate of living theory, which states that an organism's longevity is inversely related to its metabolic rate (Holliday, 1992; Comfort, 1979), suggesting that organisms with higher metabolic rates have shorter life spans. This view seems to be in line with another outdated idea, the "wear and tear" notion of aging. The validity of the metabolic rate view is now being questioned by recent revelations from several studies on homoiothermic animals, including those subject to the DR paradigm (Masoro et al., 1982).

One major finding generated from DR studies reveals that DR rats have metabolic rates similar to their non-restricted counterparts, yet have much longer life spans (Masoro et al., 1991). Similar trends are expected in primates, whose life spans are longer than predicted from their metabolic rate as calculated by oxygen intake. These findings call for a reassessment of metabolic rate as the major determinant in the aging process of all organisms (Holmes and Austad, 1995). Further, evidence casting doubts on the validity of this theory emanates from comparative studies of various species (Holmes and Austad, 1995; Barja et al.,1994). These studies reveal that birds and bats also have substantially longer life spans from what would be predicted based on their metabolic rates (Holmes and Austad, 1995; Barja et al.,1994). 
DR as a Modulator of Oxidative Stress. The possibility that nutritional intervention, other than dietary antioxidant supplementation, stave off oxidative damage was first suggested by the work of Enesco and Kurk (1981). These authors restricted dietary protein (4\% compared to $26 \%$ ) at 3, 5, 7, and 12 months of age, finding that lipofuscin, found in the brain and heart of older mice, accumulation significantly reduced. Their work was confirmed by a recent work of Youngman (1993) who demonstrated that restriction of protein intake alone can reduce oxidatively damaged DNA and other oxidative damage. The first study of DR showing its ability to suppress oxidative reactions was conducted by Chipalkatti et al. (1983). Here, the authors observed that brain lipofuscin and brain malondialdehyde is significantly lower in 50\% DR mice. Furthermore, they found that DR also reduces age-related lipid peroxidation in liver homogenate from 12- and 24-month-old mice. More recently, Sohal et al. (1994) used 40\% caloric restricted mice at 9, 17 and 23 months of age, and obtained data demonstrating reduced protein oxidation, mitochondrial State 4 respiration superoxide, and $\mathrm{H}_{2} \mathrm{O}_{2}$ production in the brain, heart, and kidney. The current perception holds that the antioxidative action of DR seems far more widespread than earlier suspected (Koizumi et al., 1987; Yu, 1993). Findings on the deleterious aging effects of membrane structure and cell function have led a number of investigators to propose the membrane hypothesis of aging. Zs-Nagy (1994) and Hansford (1983) independently hypothesized age-related declines in cellular bioenergetics based on membrane structural deterioration. This was also revealed by Grinna (1977) who documented alterations of both the gross chemical composition and the quantitation of mitochondrial and microsomal membranes in aged animals. Subsequent detailed membrane lipid analysis showed that while the major phospholipid classes did not vary significantly with age (Zs-Nagy, 1994), characteristic changes in fatty acid composition of the phospholipids did occur. Data showed that increased polyunsaturated fatty acids (20:4, 22:4 and 22:5) and decreased linoleic acid (18:2) are present in membranes from older ad libitum-fed rats. This would help explain the age-related increased susceptibility of these membranes to free radical attack. Interestingly, DR modulates a change in membrane lipid composition on the opposite direction: older DR animals have decreased in polyunsaturated fatty acid and increases in 18:2 concentration compared to their younger 
counterparts Thus, it appears that two major membrane properties, unsaturation to saturation ration and peroxidizability, were effectively modulated by DR to provide a well-preserved, intact membrane structure and integrity (Yu, 1994). Confirming those changes, Hegner (1980) was the first to note that membrane fatty acid profiles change with age and determined the detailed quantitative and qualitative shifts in saturated and unsaturated fatty acids, strongly suggesting that age affects the basic components to the membrane structure.

Effect of DR on Gycosylation Process. Variable effects of DR have been reported in the literature. For example, a 10\% decrease in blood glucose concentrations in DR rats (Cefula et al., 1995) decreases the glycation of proteins by 18-33\%. A similar observation was noted in a separate study; an approximately $11 \%$ decrease in glucose in DR animals was associated with a 34\% reduction in glycated $\mathrm{Hb}$ (Masoro, et al.,1989). Although the reason for this is unknown, a decrease in the level of oxidative stress in birds (DR as compared to AL) limit the formation of glycoxidation products by increasing the formation of antioxidant enzymes (Younman et al., 1982; Yu et al.,1982;Yu, 1994), this limitation being present in spite of higherthan-mammalian basal temperatures and concentrations of glucose. Further, strengthening the antioxidant defenses of diabetics by dietary supplementation of Vitamins $\mathrm{C}$ and $\mathrm{E}$ has been reported to decrease protein glycation without an effect on glycemia (Ceriello et al., 1991; Aoki et al., 1992; Davie et al., 1992). These observations suggest that a reduction in oxidative stress in DR animals contributes to the observed decrease in protein glycation. This might result from changes in ascorbate homeostasis or the ratio of Vitamin $\mathrm{E}$ to polyunsaturated lipid concentrations in DR compared to AL animals (Cefalu et al., 1995). While several studies have established that collagen glycosylation is increased with elevated concentrations of glucose both in vivo and in vitro and that this glycosylation increases with age in several tissues, other studies have failed to demonstrate anything more than a loose relationship between crosslinking and glycosylation, either in vivo or in vitro (Guitton et al., 1984; Le pape et al., 1984; Lyons et al., 1991; Fu et al.,1994; Monnier et al., 1996). Furthermore, Beuchat and Chong (1997) reported that, in spite of circulating concentrations of plasma glucose in excess of $650 \mathrm{mg} / \mathrm{dl}$, the amount of glycated $\mathrm{Hb}$ in hummingbirds (2-5\%) is lower as compared to mammals which have levels 
ranging from 6-8\%. These data suggest that class aves may also have a more efficient antioxidant system which limits the generation of glycoxidation products. Indirect evidence to support this view includes the observation that, in spite of an increased metabolic rate, the production of oxygen free radicals in avian (Red-tailed hawk and chicken) heterophils was significantly lower than in analogous bovine neutrophils (Conlon et al., 1991). In another study comparing pigeons and rats, researchers found that mitochondrial generation of both oxygen free radicals and peroxides was lower in pigeons and that pigeons had higher antioxidant activities (Ku and Sohal, 1993). Antioxidants such as Vitamin A (retinol and retinyl esters) and $\alpha-$ tocopherol (Vitamin E) have been found in higher concentrations in birds than in mammals (Schweger et al., 1991).

Role of Uric Acid in Birds. Uric acid is one of the circulating antioxidants that demonstrates a positive correlation with maximum life span across species (Ames et al., 1981; cultler, 1984). Humans, the most long lived among primates, have comparably high levels of uric acid because they lack uricase, the terminal degradative enzyme present in monkeys and other mammals (Ames et al., 1981; Schreiber et al., 1986). In support of this concept, the lower levels of uric acid in macaques (3-fold as compared to humans) correlate with a 3-fold shorter life span as compared to humans (Short et al., 1997). Uric acid not only demonstrates a stabilizing influence on ascorbate in human blood (Sevanian et al.,1991), by enhancing its role as a free radical scavenger (Ames et al., 1981; Cultler, 1984), but also functions as a noncatalytic binder of iron and iron-containing compounds that would otherwise catalyze the oxidation of ascorbate. This being the case, many species of birds must far surpass mammals in their ability to cope with free radical damage.

Uric acid has been proposed as a potent scavenger of free radicals in human and animal tissues (Becker, 1993; Hellsten et al., 1997). Uric acid is ubiquitous; it is found in all types of extracellular fluids including the lymphatic, CSF, interstitial, synovial, intraocular, and amniotic fluids as well as in the lining of the respiratory tract (Becker, 1993). In fact, a reduction in uric acid concentrations is associated with an increase in reperfusion injury (infarct extension) in 
humans following myocardial infarction (Parmely et al., 1992). Urate demonstrates, in vitro, the ability to scavenge peroxides, various hydroxyl radicals, and hypochlorous acid (Becker, 1993; Hellstein et al., 1997). Concentrations of plasma uric acid in birds are approximately two fold greater than the urate concentrations measured in humans (Bishop et al., 1992; Shapiro et al., 1997). Although several different antioxidants are present in the body system of birds, we suggest that uric acid may play an important role in coping with oxidative stress and formation of glycoxidation products (Klandorf et al., 1998).

Pharmacological Intervention of AGE Accumulation. Pharmaceutical intervention would seem to be the most likely route and pharmacological agents were sought that could inhibit this process by selectively blocking reactive carbonyl group on ketoamine products as well as and on their derivatives such as 3-deoxyglucosone and glycolaldehyde. Different chemical agents have been tried to delay the AGEs formation of crosslinks. Some of these include: a) Aminopyrazoles; b) Aminosubstituted benzimidazoles; c) 1,2 Disubstituted benzimidazoles; d) Substituted 1,2,4-trizoles; e) Amino-benzoic acids and derivatives; f) 2 alkylidene-aminoguanidines and derivatives; g) Aminoguanidine (AG); h) Rutin; I) Vit. $\mathrm{B}_{6}$ (pyridoxine); j) Vit. $\mathrm{B}_{1}$ (thiamine pyrophosphate); k) Vit C; 1) 1,25 (OH) ${ }_{2}$ or Vit $\mathrm{D}_{3}$ m) ALT 462 and $486,711$.

The nucleophilic hydrazine compound aminoguanidine- $\mathrm{HCl}$ has been the most extensively investigated compound of this type and effectively inhibits the formation of AGEs in vitro and in vivo (Brownlee et al., 1986). However, in some case AG did not work effectively and the precise mechanism of action is still controversial (Booth et al., 1997).

Aminoguanidine (AG). It has been proposed as an agent of potential benefit in prophylaxis of diabetic complications of kidney, nerve and eye (Brownlee et al., 1986; Brownlee et al.,1988; Hammes et al., 1991; Kumari et al., 1991; Edelstein and Brownlee,1992 a; Edelstein and Brownlee, 1992 b). In vivo, AG has been shown to diminish the formation of collagenlinked fluorescence associated with the production of AGEs. AG has been postulated to block 
pathological tissue alterations via inhibition of deleterious long-term changes to proteins associated with hyperglycemia (Edelstein and Brownlee, 1992). AG has been referred to as a "virtually non-toxic nucleophilic hydrazine $\left(\mathrm{LD}_{50}=1800 \mathrm{mg} / \mathrm{kg}\right.$ in rodents (Edelstein and Brownlee, 1992 a b).

Mode of Action of $\boldsymbol{A G}$. Numerous studies have demonstrated that the AG is an inhibitor of many manifestations of nonenzymatic glycation (Brownlee et al., 1988; Khatan et al., 1988; Odetti, 1990; Matuieu et al., 1995; Klandorf et al., 1996; Kochakian et al., 1998), some studies did not find it useful at all (Oxlund and Andreassen, 1992; Crijns et al., 1998). However, there is a disagreement over its mode of action as well ( Booth et al., 1997).

The primary mechanism by which AG inhibits the formation of AGE has not been determined. However, this small molecule, in the class of compounds called hydrazines (containing an active $\mathrm{NH}_{2}$ group), reacts with Amadori products. It binds to carbonyl groups and in so doing prevents the Amadori products from becoming AGEs (Fig. 2.9) (Brownlee et al., 1986; Oxlund and Andreassen,1992). The controversy arose after the recent kinetic studies carried out by Booth et al., 1997. The kinetic studies confirmed that AG is a potent inhibitor of antigenic AGE formation induced by high glucose concentrations (Booth et al., 1996). However, the inhibition unexpectedly appeared to diminish at later stages of the kinetics studies i.e., 144 hrs of incubation with ribose (Booth et al., 1996). Due to the slowness of the glycation with glucose, this surprising observation could not be completely established in earlier studies. Furthermore, the basis for this diminished inhibition is open to question due to the possible long term instability of aminoguanidine as well as its potential to produce hydrogen peroxide (Ou and Wolff, 1993). The kinetics of aminoguanidine inhibition of ribose glycation appear fully consistent with the earlier glucose findings. Ribose glycation results clearly demonstrate that AG slows the rate of antigenic AGE formation in the presence of sugar but has little effect on the final amount of AGE formed. In the case of glycation by ribose, the overall rate of AGE formation has been shown to be strongly dependent on ribose concentrations (Khalifah et al., 1996). In terms of the classical pathway of glycation, AG decreased the rate of initial Schiff 
base formation by complexing the acyclic free aldehyde forms of sugars (Ledl, 1990). Inhibition through formation of such an AG-sugar interaction was first advanced by Khatami et al., (1988). In addition, a glucose-AG adduct was recently produced, isolated, and structurally characterized by Hirsch et al., (1995). The hypothesis that AG does not significantly react with Amodori or later intermediates on the generation of AGEs could be directly tested by observing its possible

effects on post-Amadori kinetics, i.e., starting with protein Amadori intermediates. Preliminary studies had indicated a limited interaction of AG with post-Amadori AGE formation RNase (Khalifah et al., 1996). These results are especially noteworthy, since AG was originally proposed by its discoverers to be a nucleophilic blocker of Amadori glycation intermediates (Brownlee et al., 1986; Brownlee et al., 1988).

A novel hypothesis has recently been proposed that AG is a inhibitor of inducible NO synthase which is responsible for nitric oxide (NO) synthesis from L-arginine, as a free radical NO mediates the destruction of pancreatic $\beta$-cell in IDDM. Because AG delayed the onset diabetes, this data suggested an important role for NO in $\beta$-cell destruction. Finally, macrophages, which have been implicated in $\beta$-cell destruction, have been shown to increase NO production in diabetes-prone BB rats (Wu, 1995). So it was hypothesized that NO production may be enhanced in IDDM subjects before the onset of diabetes and that inhibition of NO production may prevent of delay the onset of diabetes.

\section{Poultry as an Animal Model for the Study of Aging}

Class aves offers unique advantage as an animal model for the study of biogerontology, particularly for the study of retarded aging and long life (Holmes and Austad, 1995). The chief features of this class relevant to aging research is that most species are dramatically longer-lived than mammals of equal body size (Lindstedt and Calde, 1976). The longevity of birds is somewhat surprising, since they exhibit several other traits which some modern theories of aging suggest should render them more susceptible to the degenerative processes of aging. These traits are (1) metabolic rates as much as 2-2.5 times as high as those of mammals of similar body sizes (Lindstedt and Calde, 1976; Austad and Fischer, 1991) which presumably expose them to a 
higher rate of free oxygen radical production and, consequently, accelerated tissue damage (Harman, 1956; Del Maestro, 1980). (2) blood sugar levels typically 2-6 times as high as those of mammals, along with a comparatively elevated body temperature (about $3^{\circ} \mathrm{C}$ higher than mammals) (Holmes and Austad, 1995). Both of these latter factors should accelerate formation of advanced Maillard products, also hypothesized to be involved in aging-related tissue degeneration (Cerami, 1985) in conjunction with the synergistic effects of free radical products (Sohal and Allen, 1990; Holmes and Austad, 1995). Keeping in view the longevity of birds, they must have special mechanism to prevent the formation of such products, degrade them once formed, remove affected cells, or some combination of the three to live longer (Holmes and Austad, 1995; Monnier, 1990; Iqbal et al., 1997).

Although poultry is an atypical representative of class aves, however, chicken can be effectively used to study its specialized protective mechanism against free radical production and Maillard reaction damage and subsequent AGEs formation, and may offer insight into medical intervention for retarding aging in general and increasing the productive period of poultry and other animals in particular. 


\section{Chapter 3}

The results of this research are presented as four separate experimental areas. They are formatted in the style of journals in which they will appear.

This chapter covers:

- Materials and Methods

- Results

- Discussion 


\title{
Study I
}

\section{Protein Glycosylation and Advanced Glycoslyated Endproducts (AGEs) Accumulation: An Avian Solution?}

\begin{abstract}
The objectives of this study were to determine the effect of diet restriction (DR) and the crosslinking inhibitor, aminoguanidine (AG), on oxidative stress, concentrations of uric acid, and the rate of pentosidine accumulation in the skin (Ps) of naturally hyperglycemic broiler breeder hens. Female chicks $(n=450)$ were randomly assigned to four groups from 8 to 92 weeks after hatch: ad libitum (AL); diet restricted (DR); AL and DR groups supplemented with 400 ppm AG each $(A L+A G$ and $D R+A G)$. The accumulation of Ps in AL birds increased linearly with age $(\mathrm{P}<0.001)$ and was significantly retarded in all treatment groups $(\mathrm{P}<0.001)$. Ps in the AL+AG group was comparable to that in the DR group, while Ps was lowest in the DR+AG group $(\mathrm{P}<0.001)$. PMA-induced respiratory bursts in blood leukocytes were lower and uric acid higher in both the $\mathrm{DR}$ and $\mathrm{DR}+\mathrm{AG}$ groups as compared to $\mathrm{AL}$ and $\mathrm{AL}+\mathrm{AG}$ groups $(\mathrm{P}<0.001)$. We concluded that the accumulation of Ps can be retarded by DR and AG.
\end{abstract}

\section{INTRODUCTION}

Class aves offers many advantages in providing animal models for biogerontology, particularly for the study of retarding aging and promoting longevity (1). The preeminent feature of this class in regard to aging is that most species within it are dramatically longer lived than mammals of comparable body size (2). Avian longevity is somewhat surprising due to other, inherently avian, traits which, based on many theories of aging, should render them more susceptible to the degenerative processes of aging. These traits include: 1: metabolic rates as much as 2 - 2.5 times higher than similarly-sized mammals $(2,3)$, which should, presumably, 
expose them to a higher rate of oxygen free radical production and, consequently, accelerated tissue damage (4-6); 2: concentrations of plasma glucose typically 2-6 times mammalian norms, which should accelerate the Maillard reaction and generate high concentrations of tissue crosslinks or advanced glycosylated endproducts (AGEs); and 3: an elevated basal body temperature (about $3^{\circ} \mathrm{C}$ higher than mammals) (1), which should contribute to the nonenzymatic attachment of glucose to proteins. Both of these latter factors should accelerate the formation of advanced Maillard products and, hence, the process of tissue aging (1).

The glycation theory of aging suggests that the modification of proteins by glucose and the associated browning or Maillard reactions leads to the gradual crosslinking, polymerization, browning, and fluorescence changes in collagen that are characteristic of aging $(7,8)$. It is postulated that, ultimately, these crosslinks will lead to the structural and functional deterioration of tissues (8-10). One such crosslink, or AGE, pentosidine, has been isolated and characterized. It is an imidizole [4, 5b] pyridinium molecule comprised of lysine and arginine residues crosslinked by a pentose (11). In mammals, pentosidine has been found to increase linearly over the lifespan of the animal and, hence, has been used as a biomarker for aging studies (12-14).

According to a synergistic theory of aging (15), age-related deterioration of tissues is due to the interplay of free radical damage, glycosylation and other Maillard reactions. The specific in vivo carbohydrate moiety which leads to pentosidine is not known, but oxidative reactions are required at some stage in its formation. In support of this view, the generation of pentosidine is inhibited in the absence of oxygen, a fact that prompted Baynes (1991) (16) to coin the term "glycoxidation product" to describe pentosidine and other glycation compounds similarly affected by oxygen availability. Glycoxidation products are exceptionally sensitive indicators of oxidative stress due to the relative ease of oxidation of both reducing sugars and their adducts. Although pentosidine accounts for only a small fraction of the carbohydrate adducts and crosslinks formed nonenzymatically in protein, its measurement is useful as a biomarker of both the glycative and oxidative damage to proteins (17). 
In contrast to the proposed progeric role of glycation and free radical formation, diet restriction (DR) has been shown to retard the aging process. In rodents, DR lowers concentrations of plasma glucose, increases longevity, decreases age-associated physiological changes, and delays or prevents many age-associated pathologies $(18,19)$. In addition to diet restriction, supplementing the diet with crosslinking inhibitors may have a positive effect on preventing age-related complications. The decrease in tissue functionality associated with nonenzymatic crosslinks can be delayed or somewhat ameliorated by supplements such as the nucleophilic hydrazine, Aminoguanidine Hydrochloride (AG) (20-23). While numerous studies have demonstrated that AG is an inhibitor of many manifestations of nonenzymatic glycation, its specific mode of action is controversial (24).

The objectives of this study were two-fold: 1: to determine the effect of diet restriction (DR) as well as the crosslinking inhibitor, aminoguanidine (AG), on the rate of accumulation of the glycoxidation product, pentosidine, in the skin (Ps) of naturally hyperglycemic broiler breeder hens, and 2: to determine the plasma concentrations of the antioxidant, uric acid, and oxidative stress in blood leukocytes. Any reduction in oxidative stress and/or the accumulation of Ps and/or an increase in uric acid could be interpreted as a reduction or slowing of the aging process.

\section{MATERIALS AND METHODS}

Birds and Management. Day-old female broiler-breeder (CobbXCobb) chicks ( $n=450)$ were placed in electrically heated battery brooders and fed ad libitum until 4 wks of age. All birds were then fed a restricted diet $(60 \%$ of $\mathrm{AL})$ which optimizes production performance. A restricted diet is limited in calories only; all diet restricted birds receive the recommended amounts of vitamins, minerals and nutrients. Feed intake of AL-fed birds was determined on a weekly basis and adjustments in feed allocation to the DR birds were made accordingly. Photoperiod was set according to the Cobb Management Guide (Cobb-Vantress, Inc., Siloam Springs, AR 72761). All chicks were assigned randomly at 4 wks of age to one of four experimental groups: ad libitum (AL); diet restricted (DR); AL and DR supplemented in feed 
with 400 ppm AG (1.35 mg/kg/day) (Aldrich Chemical Co., Inc., Milwaukee, WI 53233) (AL+AG and DR+AG, respectively). At this dose, a significant reduction in tissue fluorescence was recorded in poultry (25). Once assigned to a dietary group, the birds were fed accordingly throughout the study. All birds were fed daily between 0800 and 1000 hours and provided water ad libitum. The flocks were reared in floor pens until $20 \mathrm{wks}$ of age at which time the hens were put into individual cages. Body weight was monitored weekly and recorded every 12 wks.

Skin samples and pentosidine determination. Hens $(n=5)$ were randomly selected from each dietary group every 12 wks beginning at 8 wks of age and ending at 92 wks of age and killed by prior electrical stunning. Approximately $1 \mathrm{~g}$ of skin was removed from the abdominal area, washed with normal saline and stored at $-80^{\circ} \mathrm{C}$ until assayed. The collagen digest for pentosidine determination was prepared according to a technique for $10 \mathrm{mg}$ skin described by Monnier et al. (1986) (26) and Sell et al. (1992) (27). Briefly, this technique involved the removal of the epidermal and adipose layers, freezing in liquid nitrogen, and mincing. The minced samples were delipidated overnight in a chloroform-methanol (2:1) solution. Samples were then rehydrated in $50 \%$ methanol and hydrolyzed in $6 \mathrm{~N} \mathrm{HCl}$ at $110^{\circ} \mathrm{C}$ for $18 \mathrm{~h}$. All tubes were flushed with $\mathrm{N}_{2}$ prior to capping for heating. Subsequent to the hydrolysis, the samples were placed into a SpeedVac centrifuge-type vacuum drier (Savant Instruments, Farmingdale, NY 11735) until dry. Samples were then reconstituted in $250 \mu 1$ ddi $\mathrm{H}_{2} \mathrm{O}$ and filtered using a Costar ${ }^{\circledR}$ Spin-X® centrifuge tube filter (Corning Costar Corp., Cambridge, MA 02140). A modified Stagman and Stalder method was used to estimate collagen via an hydroxyproline standard; it was assumed that hydroxyproline made up 14\% of the total collagen (28).

The estimation of pentosidine was done by reverse phase HPLC (29). One mg of acidhydrolyzed skin collagen digest in $100 \mu \mathrm{l}$ water/0.01M heptafluorobutyric acid (HFBA) was injected into a 0.46X25 cm Vydac 218TP104 (10 $\mu \mathrm{m})$ C-18 column (Vydac, Hesperia CA 92345) connected to a Shimadzu HPLC (Shimadzu, Inc., Columbia MD 21040). This apparatus consisted of two LC-600 pumps, an SIL-6B autoinjector, RF-551 fluorescence detector (excitation $325 \mathrm{~nm}$, emission $370 \mathrm{~nm}$ ). Separations were achieved by application of a linear 
gradient of $12-42 \%$ acetonitrile from 0 to $20 \mathrm{~min}$ in water and HFBA. Quantification of pentosidine was made by comparison of peak areas with a pentosidine standard (Vincent Monnier, Cleveland, $\mathrm{OH}$ 44120) injected under identical conditions. A software package (Shimadzu CLASS-VP 4.2) was used to analyze the data.

Plasma glucose and uric acid determination. Blood samples $(\mathrm{n}=5)$ were collected from the wing vein of birds randomly selected for sacrifice as described above. Glucose was analyzed by a YSI 2700 Select biochemistry analyzer (YSI, Inc., Akron, OH 45387). Uric acid was determined using a kit from Sigma (procedure \# 685, Sigma Diagnostics, MO, 63178).

Leukocyte isolation and chemiluminescent assay for oxidative stress. One milliliter of blood from 125 wk old hens ( $\mathrm{n}=5$ per group except for the AL group where only 2 birds remained) was suspended in mono-poly resolving medium (ICN 16-980-49) and leukocytes were isolated by centrifugation. The total number of leukocytes were counted using a routine hemocytometric technique. To a $3 \mathrm{ml}$ luminometer tube were then added $100 \mu 1$ of leukocytes, $100 \mu 1$ luminol solution, $200 \mu \mathrm{l}$ PBS, and $100 \mu 1$ phorbol myristate acetate (PMA). The luminometer tube was placed into a luminometer (Berthold model LB 9505C) with the temperature control set at $37^{\circ} \mathrm{C}$. Oxidative activity was determined by measuring the luminescence generated over $30 \mathrm{~min}$; results were reported as counts per minute (CPM). The data was analyzed by a PC running KINB software. Luminescence was corrected for each group based on the number of leukocytes present.

\section{Statistical Analyses}

Data were analyzed by the general linear models procedure using a $2 \times 2 \times$ time factorial design (30). Regression analysis was performed to determine the correlation between the concentration of Ps and age. Oxidative stress was analyzed by a one-way ANOVA. The Student-Newman-Keuls Range method was used to test the significance of the difference between means.

\section{RESULTS}




\section{Plasma Glucose and Body Weight}

Hens in the DR groups weighed less at all ages monitored than those in the AL groups $(\mathrm{P}<0.0001$; Fig. 1). At wks 44 and $68 \mathrm{DR}$ increased $(\mathrm{P}<0.05)$ concentrations of plasma glucose as compared with AL birds (Fig. 2). AG did not affect body weight or plasma glucose concentrations.

\section{Skin Pentosidine (Ps)}

Ps accumulated linearly with age in AL-fed birds $\left(\mathrm{r}^{2}=0.7768\right)$; DR significantly reduced its accumulation in broiler breeder hens over the 92 weeks $(\mathrm{P}<0.001$; Fig. 3). Concentrations of Ps in DR birds increased initially after hatch and then plateaued. For the AL group, concentrations of Ps were $0.018 \pm 0.001 \mathrm{pmol} / \mathrm{mg}$ collagen for $8 \mathrm{wk}$ old birds and $0.171 \pm 0.018$ $\mathrm{pmol} / \mathrm{mg}$ collagen at $92 \mathrm{wks}$ of age. Concentrations in the DR group were $0.009 \pm 0.0007$ $\mathrm{pmol} / \mathrm{mg}$ collagen and $0.069 \pm 0.008 \mathrm{pmol} / \mathrm{mg}$ collagen for 8 and $92 \mathrm{wks}$ of age, respectively. Dietary supplementation with AG lowered the rate of Ps accumulation; concentrations of Ps in the AL+AG group were comparable to those of the DR group (Fig. 3). Aminoguanidine reduced $(\mathrm{P}<0.001)$ the accumulation of Ps in both dietary groups. The AL+AG group had concentrations of Ps ranging from $0.001 \pm 0.0009 \mathrm{pmol} / \mathrm{mg}$ collagen at 8 wks to $0.049 \pm 0.005 \mathrm{pmol} / \mathrm{mg}$ collagen at $92 \mathrm{wks}$, while the concentrations in the DR+AG group ranged from $0.012 \pm 0.002$ $\mathrm{pmol} / \mathrm{mg}$ collagen at $8 \mathrm{wks}$ to $0.036 \pm 0.003$ at $\mathrm{pmol} / \mathrm{mg}$ collagen at 92 wks.

\section{Oxidative Stress and Uric Acid}

PMA-induced respiratory bursts as a measure of oxidative stress were lower $(\mathrm{P}<0.0001)$ and plasma uric acid higher $(\mathrm{P}<0.0003)$ in both the $\mathrm{DR}$ and $\mathrm{DR}+\mathrm{AG}$ groups than in the $\mathrm{AL}$ and AL+AG groups $(\mathrm{P}<0.001)$. The AL+AG group showed a $10 \%$ increase in uric acid (Fig. 4) and a $302 \%$ decrease in oxidative stress (Fig. 5) as compared to the non-supplemented AL group. Plasma uric acid remained high at most time points and an overall increase in its mean concentrations was evident in DR-fed birds (Fig. 4). DR lowered oxidative stress and AG further enhanced that effect (Fig. 5). 


\section{DISCUSSION}

Contrary to expectations, the higher-than-mammalian concentrations of glucose in AL birds were not associated with higher-than-mammalian concentrations of Ps. In fact, Ps concentrations in the hens $(0.171 \pm 0.018 \mathrm{pmol} / \mathrm{mg}$ collagen at 92 wks of age for AL-fed birds $)$ were markedly lower than those reported in mammals $(29 \mathrm{pmol} / \mathrm{mg}$ for human skin collagen and $10 \mathrm{pmol} / \mathrm{mg}$ for bovine tendon collagen (commercial Type I insoluble collagen)) (11). Thus, the formation of pentosidine, and, hence, possibly other AGEs, may not solely depend on high concentrations of plasma glucose per se, even in the presence of elevated basal temperatures and metabolic rates, as found in birds. In fact, glycosylated collagen may stimulate the oxidation process and lead to pentosidine formation (31) although only $0.4-20 \%$ collagen by weight is reported to be glycosylated $(32,33)$. In addition, whether the chemical composition of avian collagen with respect to arginine/lysine is responsible for reduced crosslink formation as compared to mammals remains to be established. The accumulation of Ps in AL birds was linear over the 92 wk sampling period (Fig. 6), comparable to the results reported in mammals (shrew, monkey, dog, pig, cow, rat, human) $(11,34)$.

One of our most interesting results is the finding that, despite the fact that DR reduced concentrations of Ps, it did not produce a corresponding reduction in concentrations of plasma glucose. In this study, in contrast to the prediction of the glycosylation theory of aging, which states that glucose or other products of carbohydrate metabolism are important contributors to the aging process so that DR (and a corresponding reduction in concentrations of plasma glucose) would be expected to reduce tissue protein glycosylation and AGE accumulation, DR birds had decreased concentrations of Ps $(\mathrm{P}<0.001)$ coupled with increased average concentrations of plasma glucose $(\mathrm{P}<0.05)$ when compared to AL birds.

Variable effects of DR have been reported in the literature. For example, a 10\% decrease in blood glucose concentrations in DR rats (14) decreased the glycation of proteins by 18-33\%. A similar observation was noted in a separate study; an approximately $11 \%$ decrease in glucose in DR animals was associated with a $34 \%$ reduction in glycated $\mathrm{Hb}(35)$. The reason for this is 
unknown, however a decrease in the level of oxidative stress in birds (DR as compared to AL) may limit the formation of glycoxidation products by increasing antioxidant enzymes (36-38), this limitation being present in spite of higher-than-mammalian basal temperatures and concentrations of glucose. Strengthening the antioxidant defenses of diabetics by dietary supplementation of Vitamins $\mathrm{C}$ and $\mathrm{E}$ has been reported to decrease protein glycation without an effect on glycemia (39-41). These observations suggest that a decrease in oxidative stress in DR animals contributes to the observed decrease in protein glycation. This might result from changes in ascorbate homeostasis or the ratio of Vitamin E to polyunsaturated lipid concentrations in DR compared to AL animals (14). In the present study, a $4 \%$ increase in mean overall plasma glucose concentration was associated with a $99 \%$ decrease in mean Ps concentration. While several studies have firmly established that collagen glycosylation is increased with elevated concentrations of glucose both in vivo and in vitro and that this glycosylation increases with age in several tissues, other studies have failed to demonstrate anything more than a loose relationship between crosslinking and glycosylation, either in vivo or in vitro (42-46). Furthermore, Beuchat and Chong (1997) (47) reported that, in spite of circulating concentrations of plasma glucose in excess of $650 \mathrm{mg} / \mathrm{dl}$, the amount of glycated $\mathrm{Hb}$ in hummingbirds (2-5\%) is lower as compared to mammals which have levels ranging from 6$8 \%$. These data suggest that class aves may also have a more efficient antioxidant system which limits the generation of glycoxidation products. Indirect evidence to support this view includes the observation that, in spite of an increased metabolic rate, the production of oxygen free radicals in avian (Red-tailed hawk and chicken) heterophils was significantly lower than in analogous bovine neutrophils (48). In another study comparing pigeons and rats, researchers found that mitochondrial generation of both oxygen free radicals and peroxides was lower in pigeons and that pigeons had higher antioxidant activities (49). Antioxidants such as Vitamin A (retinol and retinyl esters) and $\alpha$-tocopherol (Vitamin E) have been found in higher concentrations in birds than in mammals (50).

Uric acid is one of the circulating antioxidants that demonstrates a positive correlation with maximum life span across species $(51,52)$. Humans, the most long lived among primates, 
have comparably high levels of uric acid because they lack uricase, the terminal degradative enzyme present in monkeys and other mammals $(51,53)$. In support of this concept, the lower levels of uric acid in macaques (3-fold as compared to humans) correlate with a 3-fold shorter life span as compared to humans (54). Uric acid not only demonstrates a stabilizing influence on ascorbate in human blood (55), enhancing its role as a free radical scavenger $(51,52)$, it also functions as a noncatalytic binder of iron and iron-containing compounds that would otherwise catalyze the oxidation of ascorbate. This being the case, many species of birds must far surpass mammals in their ability to cope with free radical damage.

Uric acid has been proposed as a potent scavenger of free radicals in human and animal tissues $(56,57)$. Uric acid is ubiquitous; it is found in all types of extracellular fluids including the lymphatic, CSF, interstitial, synovial, intraocular, and amniotic fluids as well as in the lining of the respiratory tract (56). In fact, a reduction in uric acid concentrations is associated with an increase in reperfusion injury (infarct extension) in humans following myocardial infarction (58). Urate demonstrates, in vitro, the ability to scavenge peroxides, various hydroxyl radicals, and hypochlorous acid $(56,57)$. Because the concentrations of plasma uric acid in birds are approximately twice greater than the urate concentrations measured in humans $(59,60)$, we propose that the lower tissue concentrations of Ps in birds as compared to mammals are due to a more efficacious avian antioxidant system. Although several different antioxidants are present in the body system of birds, we suggest that uric acid may play an important role in coping with oxidative stress. However, the results from these studies do not support a predominant role for uric acid as the agent by which oxidative stress and Ps were reduced in DR and AGsupplemented birds.

The supplementation of an AL-fed animal with AG reduced the concentration of Ps to that measured in a DR animal. The reduction in concentrations of Ps in AL+AG birds was associated with a $10 \%$ overall increase in uric acid and a concomitant $302 \%$ reduction in PMAinduced respiratory bursts in blood leukocytes. On the other hand, in the DR+AG group the overall decrease in Ps was 43\%, while the AL+AG birds showed an overall increase in uric acid 
of $14 \%$ with an $85 \%$ reduction in PMA-induced respiratory bursts. DR has been shown to lower oxidative stress by modulating free radical production and increasing the concentration of antioxidant enzymes (36-38). Whether AG's mechanism of action in lowering oxidative stress mimics that of DR remains to be established. Reduced glycation as a result of reduced oxidative stress, in AL supplemented with AG as well as in DR, should increase longevity as suggested by the free radical theory of aging.

It is concluded that the rate of accumulation of Ps can be retarded by DR and AG and that birds appear able to retard the accumulation of glycoxidation products and their associated tissue damage in a more effective manner than mammals. This observation has important clinical applications in that a newly diagnosed diabetic may be able to limit or even prevent the accelerated accumulation of crosslinkages and the associated tissue degeneration. In regard to animal agriculture, the reduction in crosslinks may ultimately translate into an improvement in carcass composition and meat tenderness as well as an increase in production cycles for certain species.

\section{Acknowledgments}

The authors would like to thank Drs. Vincent M. Monnier (Dept. of Pathology, Case Western Reserve University, Cleveland, $\mathrm{OH}$ ) for supplying the pentosidine standard, Knox Van Dyke (Dept. of Pharmacology and Toxicology, West Virginia University, Morgantown, WV) for his assistance in measuring oxidative stress, and Edwin C. Townsend (Agriculture and Forestry Experiment Station, West Virginia University, Morgantown, WV) for his help with the statistical analyses. 


\section{REFERENCES}

1. Holmes, D., and Austad, S. Birds as animal models for the comparative biology of aging: A Prospectus. J. Gerontol: Biol. Sci. 1995; 50A/2:B59-B56.

2. Lindstedt, S., and Calde, W. Body size and longevity in birds. Condor 1976;78:91-94.

3. Austad, S., and Fischer, K. Mammalian aging, metabolism, and ecology: evidence from bats and marsupials. J. Gerontol: Biol. Sci. 1991;46:B47-B53.

4. Harman, D. Aging: a theory based on free radical and radiation chemistry. J. Gerontol: Biol. Sci. 1956;11:289-300.

5. DelMaestro, R. An approach to free radicals in medicine and biology. Acta Physiol. Scand. 1980;492:153-168.

6. Sohal, R., and Allen, R. Oxidative stress as a causal factor in differentiations and aging: A unifying hypothesis. Exp. Gerontol. 1990;25:499-522.

7. Cerami, A. Hypothesis: Glucose as a mediator of aging. J. Amer. Geriatr. Soc. 1985;33:626-634.

8. Monnier, V. Toward a Maillard reaction theory of aging. Prog. Clin. Biol. Res. 304, 1-22. (1989).

9. Bucala, R., and Cerami, A. Advanced glycosylation: Chemistry, biology, and implications for diabetes and aging. Adv. Pharmacol. 1992;23:1-34.

10. Vlassara, H., Bucala, R., and Striker, L. Pathogenic effects of advanced glycosylation: Biochemical, biologic, and clinical implications for diabetes and aging. Lab. Invest. 1994;70:138-151.

11. Sell, D., and Monnier V. Structural elucidation of a senescence cross-link from human extracellular matrix: Implication of pentoses in the aging process. J. Biol. Chem. 1989;264:21597-21602.

12. Grandhee, S., and Monnier, V. Mechanisms of the formation of the Maillard protein cross-link pentosidine: ribose, glucose, fructose, and ascorbate as pentosidine precursors. J. Biol. Chem. 1991;266:11649-11653.

13. Dyer, D.G., Blackledge, J.A., Thorpe, S.R., Baynes, J.W. Formation of pentosidine during nonenzymatic browning of proteins by glucose: identification of glucose and other 
carbohydrates as possible precursors of pentosidine in vivo. J. Biol. Chem. 1991b;266:11654-11660.

14. Cefalu, W.T., Bell-Farrow, A.D., Wang, Z.Q., Sonntag, W.E., Fu, M., Baynes, J.W., and Thorpe, S.R. Caloric restriction decreases age-dependent accumulation of the glycoxidation products, $\mathrm{N}$-(Carboxymethyl)lysine and pentosidine, in rat skin collagen. J. Gerontol: Biol. Sci. 1995;50A:B337-B341.

15. Kristal, B., and Yu, B.. An emerging hypothesis: synergistic induction of aging by free radicals and Maillard reactions. J. Gerontol: Biol. Sci. 1992;47:B107-B114.

16. Baynes, J.W. Perspectives in diabetes. Role of oxidative stress in development of complications in diabetes. Diabetes 1991;40:405-412.

17. Dyer, D.G., Dunn, J.A., Thorpe, S.R., and Lyons, T.J. Accumulation of Maillard reaction products in skin collagen in diabetes and aging. Annals of the NY Acad. of Sci. 1992;663:421-422.

18. Weindruch, R., and Walford, R.L. The retardation of aging and disease by dietary restriction. Springfield, IL.=: Charles C. Thomas. 1988.

19. Snyder, D.L., ed. Dietary restriction and aging: Proceedings of the symposium on the effects of dietary restriction of aging and disease in germfree and conventional LobundWistar rats. Proc. Clin. Biol. Res. 1989;289 New York: Alan R. Liss.

20. Khatan, M., Soldan, Z., David, I., Li, W., and Rockey, J.H. Inhibitory effects of pyridoxal phosphate, ascorbate, and aminoguanidine on nonenzymatic glycosylation. Life Sci. 1988;43:1725-1731.

21. Odetti, P.R., Borgoglio, A., Pascale, A.D., Polandi, R., and Adezati, L. Prevention of diabetes-increased aging effect on rat collagen-linked fluorescence by aminoguanidine and rutin. Diabetes. 1990;39:796-801L.

22. Oxuland, H. and Andreassen, T. Aminoguanidine treatment reduces increase in collagen stability of rats with experimental diabetes mellitus. Diabetologia. 1992;35a:19-25.

23. Wu, G. Nitric oxide synthesis and effect of aminonguanidine and N-monomethyl-Larginine on the onset of diabetes in spontaneously diabetic BB rats. Diabetes. 1995;44:364-365. 
24. Booth, A.A., Khalifah, R.G., Todd, P., and Hudson, B.G. In vitro kinetic studies of formation of antigenic advanced glycation end products (AGEs): novel inhibition of postAmadori glycation pathways. J. Biol. Chem. 1997;272:5430-5437.

25. Klandorf, H., Holt, S., McGowan, J., Pinchasov, Y., Deyette, D., and Peterson, R. Hyperglycemia and non-enzymatic glycation of serum and tissue proteins in chickens. Comp Biochem. Physiol. 1995;110C/2:215-220.

26. Monnier, V.M., Vishwanath, V., Frank, K.E., Elmets, C.A., Dauchot, P., and Kohn, R.R. Relation between complications of type I diabetes mellitus and collagen-linked fluorescence. N. Eng. J. Med. 1986;314:403-408.

27. Sell, D.R., Lapolla, A., Odetti, P., Fogarty, J.,and Monnier, V.M. Pentosidine formation in skin correlates with severity of complications in individuals with long-standing IDDM. Diabetes. 1992;41:1286-1291.

28. Maekawa, T., Ratinasamy, Y.K, Altman, K.I., and Forbes, W.F. Changes in collagen with age-I. The extraction of acid soluble collagens from skin of mice. Exp. Gerontol. 1970;5:177-186.

29. Iqbal, M., Probert, L.L., and Klandorf, H. Effect of aminoguanidine on tissue pentosidine and reproductive performance in broiler breeders. Poult. Sci. 1997;76:1574-1579.

30. SAS Institute. SAT/STAT User's Guide: Statistics: Release 6.04 SAS Institute Inc., Cary, NC. 1990.

31. Paul, R.G. and Bailey, A.J. Glycation of collagen: the basis of its central role in the late complications of aging and diabetes. Int. J. Biochem. Cell. Biol. 1996;28:1297-1310.

32. Bailey, A.J., and Light, N.D. The structure and growth of muscle. In: Lawrie, R.A., ed. Meat Science 5th ed. Pergamon Press, Inc., Elmsford, NY. 1989;27-47.

33. Voet, D., and Voet, J.G. Three-dimensional structure of proteins. In: Biochemistry. 1st ed. John Wiley \& Sons Inc. New York, NY. 1990;144-190.

34. Sell, D.R., Lane, M.A., Johnson, W.A., Masoro, E.J., Mock, O.B., Reiser, K.M., Fogarty, J.F., Cutler, R.G., Ingram, D.K., Roth, G.S., and Monnier, V.M. Longevity and the genetic determination of collagen glycoxidation kinetics in mammalian senescence. Proc. Natl. Acad. Sci. 1996;93:485-490. 
35. Masoro, E., Katz, M., and McMahan, C. Evidence for the glycation hypothesis of aging from the food-restricted rodent model. J. Gerontol: Biol. Sci. 1989;44:B20-B22.

36. Youngman, L.D., Park, J-YK., Ames, B.N. Protein oxidation associated with aging is reduced by dietary restriction of proteins or calories. Proc. Natl. Acad. Sci. 1992;89:9112-9116.

37. Yu, B.P., Masoro, E.J., Murafa, I., Berfrand, H.Q., and Lynd, F.T. Life-span study of SPF Fischer 344 male rats fed ad libitum or restricted diets: longevity, growth, lean body mass and disease. J. Gerontol. 1982;37:130.

38. Yu, B.P. How diet influences the aging process of the rat. Proc. Soc. Exp. Biol. Med. 1994;205:97-107.

39. Ceriello, A., Guigliano, D., Quataro, A., Donzella, C., Dipalo, G., and Lefebvre, P.J. Vitamin E reduction of protein glycosylation in diabetes. Diabetes Care 1991;14:68-72.

40. Aoki, Y. Yanagisawa, Y., Yazaki, K., Oguchi, H., Kiyosawa, K., and Furuta, S. Protective effect of vitamin E supplementation on increased thermal stability of collagen in diabetic rats. Diabetologia 1992;35:913-916.

41. Davie, S.J., Gould, B.J. and Yadkin, J.S. Effect of Vitamin C on glycosylation of protein. Diabetes 1992;41:167-173.

42. Guitton, J.D., LePape, A., Muh, J.P. Influences of in vitro non-enzymatic glycosylation on the physicochemical parameters of Type I collagen. Collagen Relat. Res. 1984;4:253264.

43. LePape, A., Guitton, J.D., Muh, J.P. Distribution of non-enzymatically bound glucose in vivo and in vitro glycosylated Type I collagen molecules. FEBS Lett. 1984;170:23-27.

44. Lyons, T.J., Bailie, K.E., Dyer, D.G., Dunn, J.A., and Baynes, J.W. Decrease in skin collagen glycation with improved glycemic control in patients with insulin-dependent diabetes mellitus. J. Clin. Invest. 1991;87:1910-1915.

45. Fu, M-X, Wells-Knect, K.J., Blackledge, J.A., Lyons, T.J., Thorpe, S.R., and Baynes, J.W. Glycation, glycoxidation and crosslinking of collagen by glucose: kinetics, mechanisms, and inhibition of late stages of the Maillard reaction. Diabetes 1994;43:676-683. 
46. Monnier, V., Glomb, M., Elgawish, A., and Sell, D. The mechanism of collagen crosslinking in diabetes: A puzzle nearing resolution. Diabetes 1996;45:S67-S72.

47. Beuchat, C.A., and Chong, C.R. (1997). Hyperglycemia in hummingbirds: Implications for hummingbird ecology and human health. FASEB J. 1997;11:3:A91.

48. Conlon, P., Smith, D., and Gowlett, T. Oxygen radical production by avian leukocytes. Can. J. Vet. Res. 1991;55:193-195.

49. $\mathrm{Ku}, \mathrm{H}$. and Sohal, R.S. Comparison of mitochondrial prooxidant generation and antioxidant defenses between rat and pigeon: possible basis of variation in longevity and metabolic potential. Mech. Aging Dev. 1993;72:67-76.

50. Schweiger, F.F., Uehlein-harrell, S., Von Hegel, G., and Wiesner, H. Vitamin A (retinol and retinyl esters), $\alpha$-tocopherol and lipid levels in plasma of captive wild mammals and birds. Xentralblatt fur Veterinarmedizin-Reihe. 1991;A38:35-42.

51. Ames, B.N., Cathcart, R., Schwiers, E., and Hochstein, P. Uric acid provides an antioxidant defense in humans against oxidant- and radical-caused aging and cancer: a hypothesis. Proc. Natl. Acad. Sci. 1981;78:6858-6862.

52. Cutler, R.G. Urate and ascorbate: Their possible roles as antioxidants in determining longevity of mammalian species. Arch. Gerontol. Geriatr. 1984;3:321-348.

53. Schreiber, G., Tiemeyer, W., Flurer, C.I., and Zucker, H. Purine metabolites in serum of higher primates, including man. Int. J. Primatol. 1986;7:521-531

54. Short, R., Williams, D.D., and Bowden, D.M. Circulating antioxidants as determinants of the rate of biological aging in Pigtailed Macaques (Macaca nemestrina). J Gerontol.: Biol. Sci. 1997;52A:B26-B30.

55. Sevanian, A., Davies, K.J.A., and Hochstein, P. Serum urate as an antioxidant for ascorbic acid. Am. J. Clin. Nutr. 1991;54:1129S-1143S.

56. Becker, F.B. Towards the physiological function of uric acid. Free Radical Biol. and Med. 1993;14:615-631.

57. Hellsten, Y., Tullson, P.C., Richter, E.A., and Bangsbo, J. Oxidation of urate in human skeletal muscle during exercise. Free Radical Biol. and Med. 1997;22:169-174. 
58. Parmley, L., Mufti, A.G., Downey, J.M. Allopurinol therapy of ischemic heart disease with infarct extension. Can. J. Cardiol. 1992;8:280-286.

59. Bishop, M.L., Duben-Engelkink, J.L., and Fody, E.P. Clinical chemistry, principles, procedures, correlations. 2nd. Ed. Philadelphia, PA: Lippincott. 1992.

60. Shapiro, F., Mahagna, M., and Nir, I. Stunting syndrome in broilers: effect of glucose or maltose supplementation on digestive organs, intestinal disaccharidase, and some blood metabolites. Poult. Sci. 1997;76:369-380. 
Figure 1: Effect of age and diet restriction (DR) and aminoguanidine (AG) on body weight (BW). Each point is the mean $(\mathrm{n}=5) \pm$ SEM. $* \mathrm{P}<0.0001$ between dietary groups (AL vs DR) at all time points.

Figure 2: Effect of age and diet restriction (DR) and aminoguanidine (AG) on concentrations of plasma glucose. Each point is the mean $(n=5) \pm$ SEM. $* \mathrm{P}<0.02$ at those time points (AL vs DR).

Figure 3: Effect of age, diet restriction (DR), and aminoguanidine (AG) on skin pentosidine (Ps). Each point is the mean $(n=5) \pm$ SEM. Comparisons were within dietary groups (AL vs AL+AG and DR vs DR+AG). $* \mathrm{P}<0.05$ and $* * \mathrm{P}<0.001$.

Figure 4: Effect of age, diet restriction (DR), and aminoguanidine (AG) on concentrations of plasma uric acid. Each point is the mean $(\mathrm{n}=5) \pm$ SEM. $* \mathrm{P}<0.05$ and $* * \mathrm{P}<0.0001$ at those time points (AL vs DR).

Figure 5: Effect of age, diet restriction, and AG on oxidative stress. Each point is the mean $(\mathrm{n}=5) \pm$ SEM, except AL $(\mathrm{n}=2)$. Groups with different letters differ significantly $(\mathrm{P}<0.0001)$.

Figure 6: Correlation between age and pentosidine accumulation in the skin of ad libitum-fed broiler breeder hens. Each point is the mean $(n=5) \pm S E M$. 


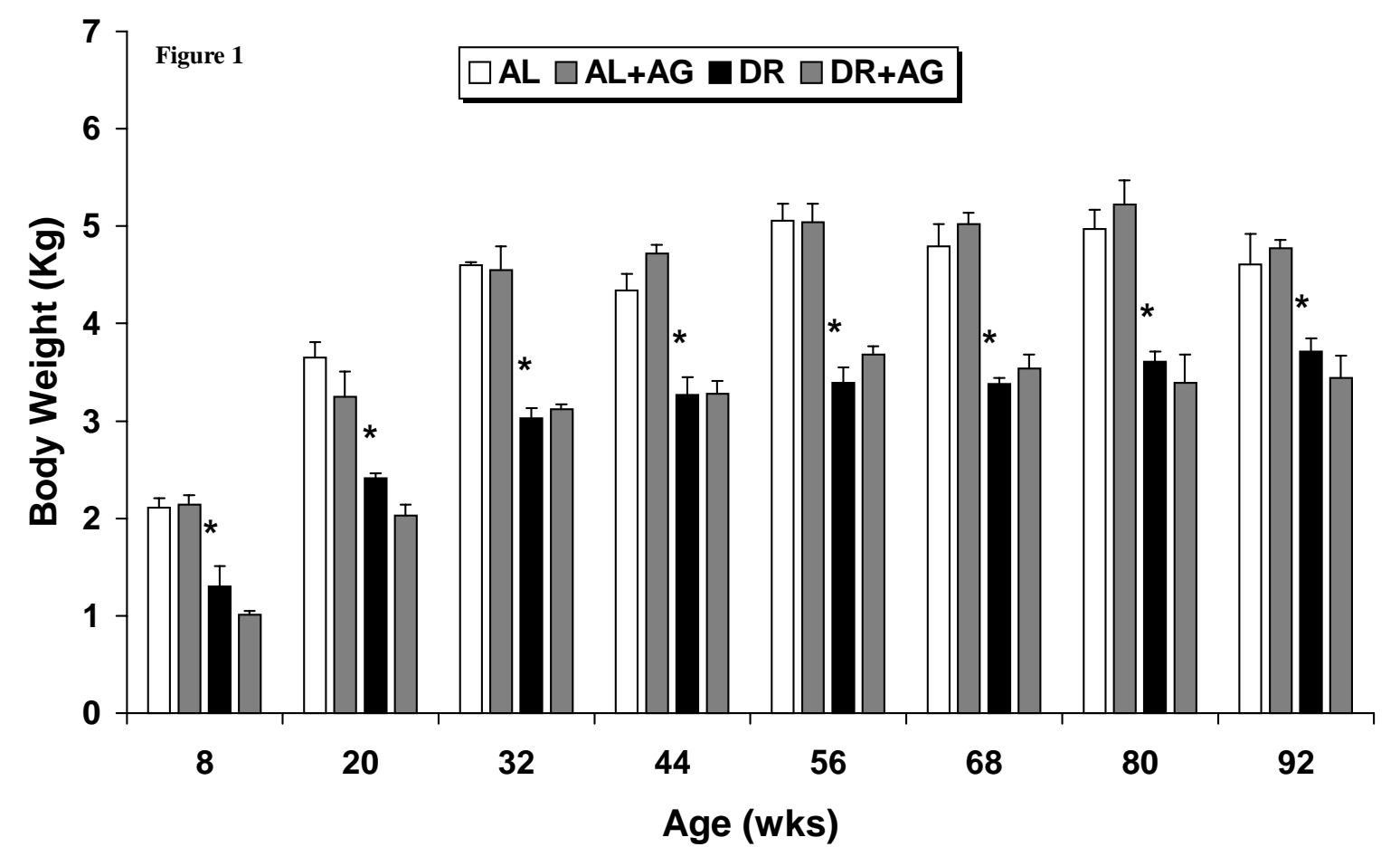




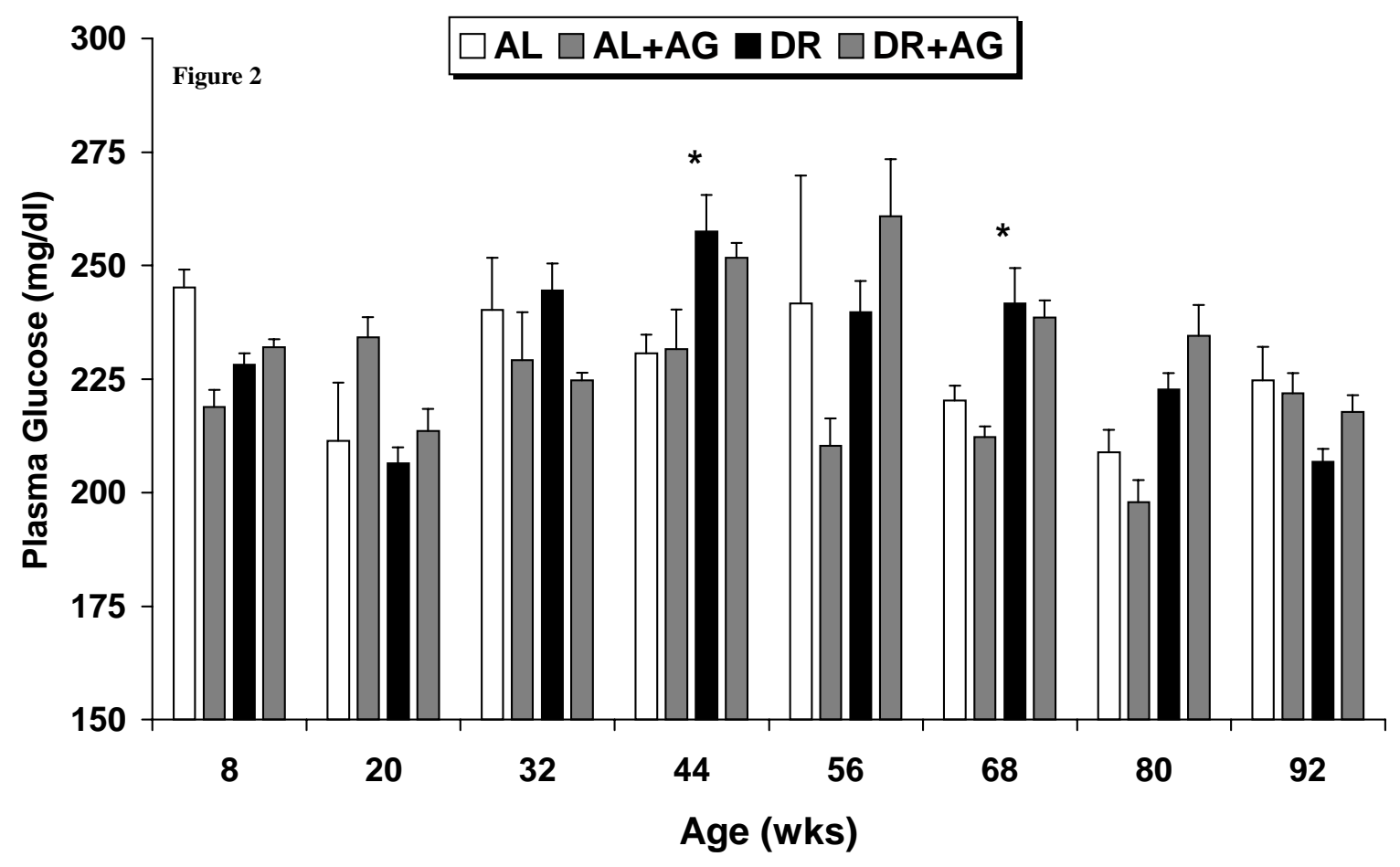




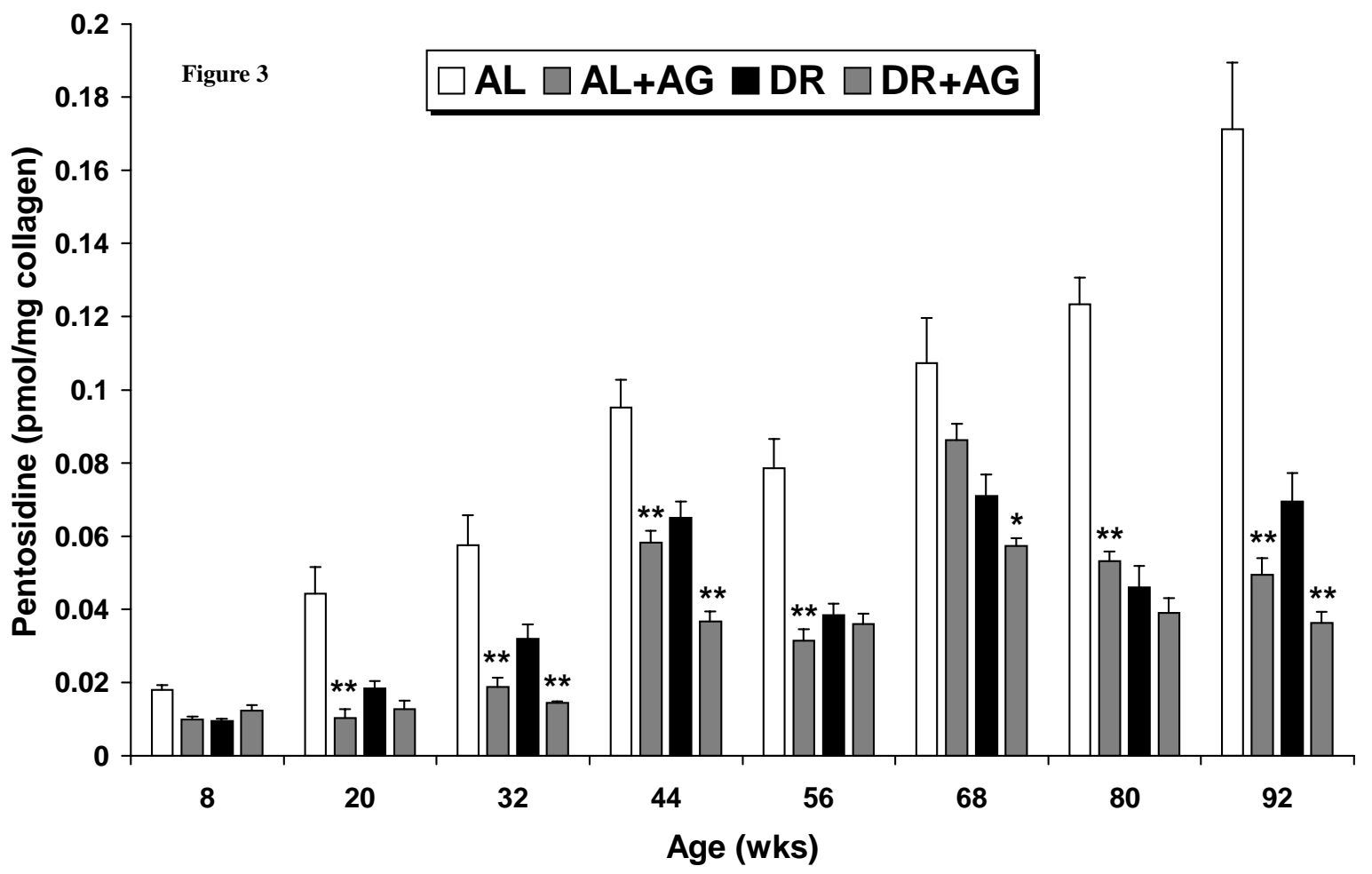




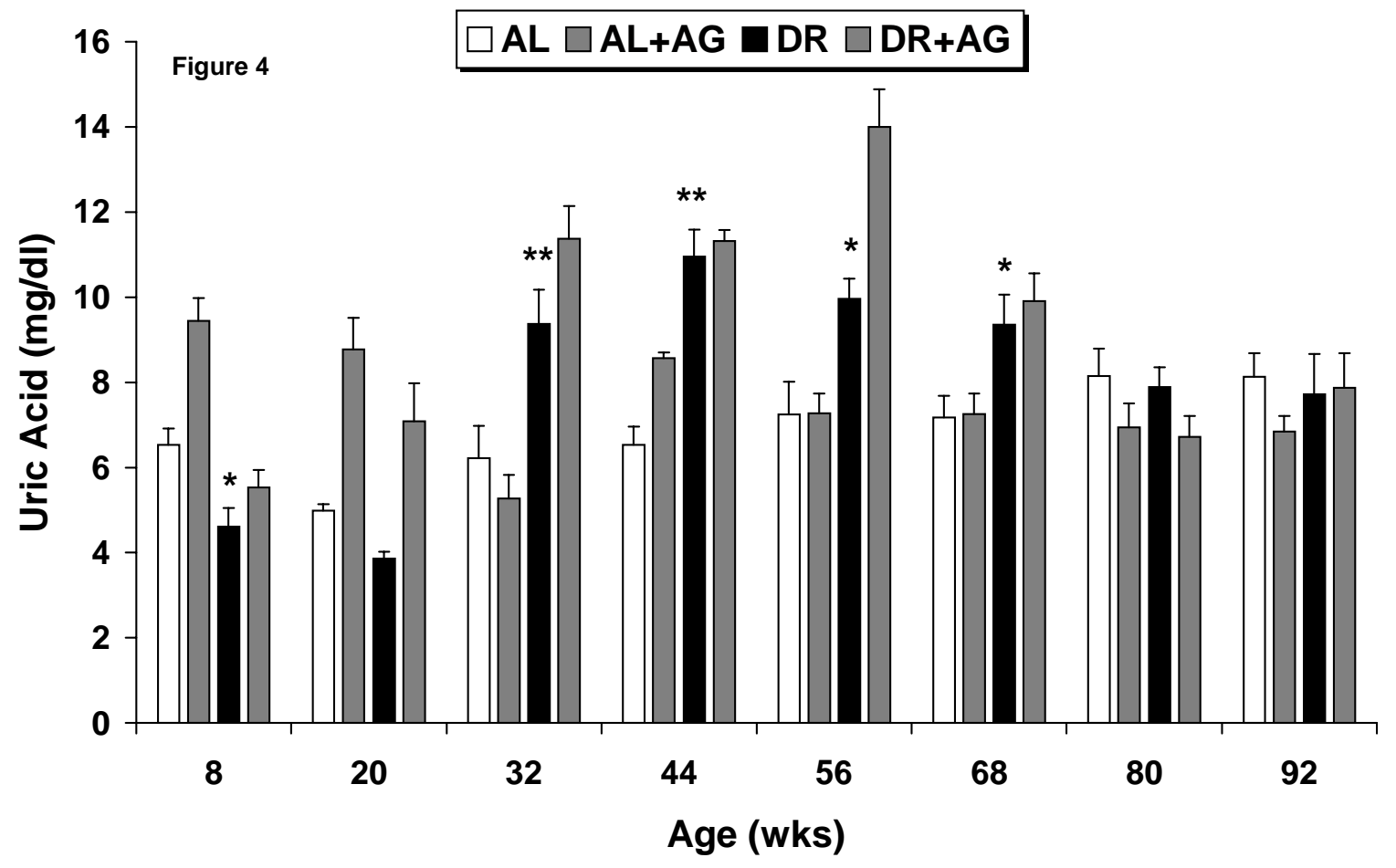


Figure 5

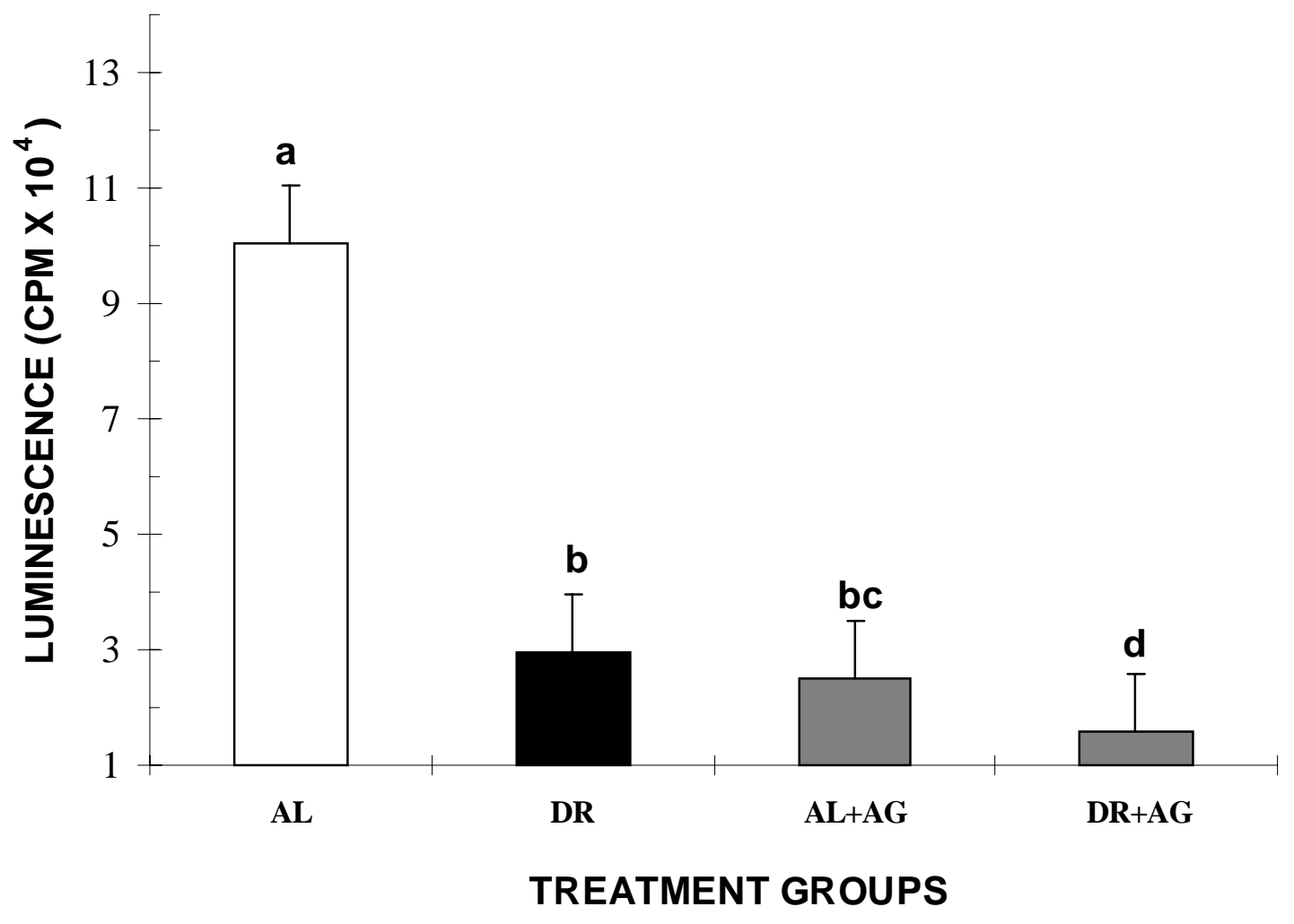




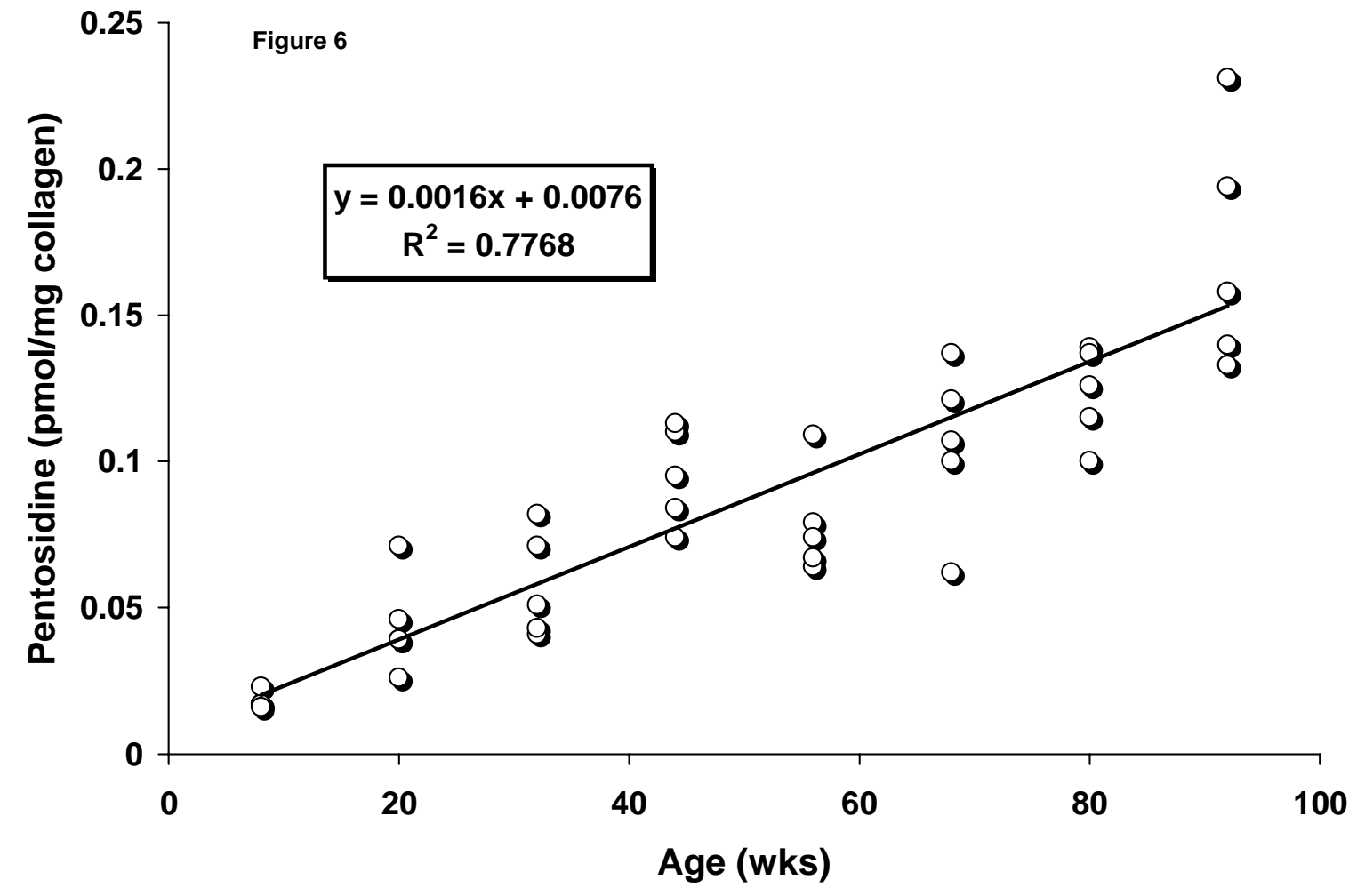




\title{
Study II
}

\section{Relationship between Mechanical Properties and Pentosidine in tendon: Effect of Age, Diet Restriction and Aminoguanidine in Broiler Breeder Hens}

\begin{abstract}
The objective of this study was to compare tendon breaking time (TBT) and tendon breaking strength (TBS) of the flexor perforans et perforatus digiti iii tendon with concentrations of pentosidine in tendons $\left(\mathrm{P}_{\mathrm{t}}\right)$ of broiler breeder hens from 8 to $125 \mathrm{wk}$ of age. In addition, effects of diet restriction (DR) and crosslinking inhibitor, aminoguanidine (AG) on $\mathrm{P}_{t}$, TBS and TBT were determined. Female chicks $(n=450)$ were randomly assigned to four treatment groups immediately after hatch: ad libitum (AL); feed diet restricted (DR; 60\% of AL); feed of AL and DR groups supplemented with $1.35 \mathrm{mg} / \mathrm{kg}$ BW/day $\mathrm{AG}$ each (AL+AG and $\mathrm{DR}+\mathrm{AG}$, respectively). In AL-fed hens $\mathrm{P}_{\mathrm{t}}$ increased with increasing age $\left(r^{2}=0.79 ; p<0.0001\right)$. Concurrently, there were age-related parallel increases in TBS $\left(r^{2}=0.56 ; p<0.0001\right)$, and TBT $\left(r^{2}=0.67 ; p<0.0001\right)$. The rate of accumulation of $\mathrm{P}_{\mathrm{t}}$ in DR group was lower $(p<0.0001)$, as were TBS $(p<0.01)$ and TBT $(p<0.02)$ compared to AL hens. Concentration of $\mathrm{P}_{\mathrm{t}}$ in AL+AG group was lower $(p<0.0002)$ as were TBS and TBT $(\mathrm{p}<0.01$ and $\mathrm{p}<0.01$, respectively). Supplementation of DR with AG did not affect $(p>0.05) \mathrm{P}_{t}$, TBS and TBT. In conclusion, the age-related increase in $\mathrm{P}_{\mathrm{t}}$ and loss of elasticity in the tendon was retarded by DR. AG was effective in AL-fed hens where $\mathrm{P}_{\mathrm{t}}$, TBT and TBS declined compared to DR-fed hens.
\end{abstract}

\section{INTRODUCTION}


Abnormally high concentrations of plasma glucose over a period of many years may result in impaired tissue function, as found in diabetic patients. The cause of these complications appears to be multifactorial (1). One of the factors may be nonenzymatic glycation with subsequent formation of browning products resulting in irreversibly crosslinked protein (2-8). These crosslinks would be expected to affect the biomechanical properties of connective tissues, because collagens are long-lived proteins that possess $\epsilon$-amino groups of lysyl and hydroxylysyl residues. Kohn (9) hypothesized that manifestations of aging are most pronounced in tissues with slow turnover, such as connective tissues of skin and tendon. In this regard, two established biomarkers of aging are rat tail tendon breaking time (TBT) (10-11) and the protein crosslink, pentosidine (12-14). Increased biomechanical strength and crosslinking of collagens were observed in vitro, after incubation of rat tail tendons with glucose $(3,15-17)$, and in vivo in animals with experimental diabetes (18-19). Verzar (20) demonstrated that resistance to thermal denaturation of rat tail tendon, measured as TBT, increased with advancing age. Further work by Everitt et al. (21-22) showed that dietary restriction initiated at an early age in rats retarded aging rate of tail tendon collagen fibers, and inhibited the development of certain agerelated disease processes such as renal disease, cardiac enlargement, and tumors. Further, Harrison et al. (23) and Harrison and Archer (24) established a strong correlation between age and TBT in inbred and hybrid strains of mice.

Since solubility of tail tendon collagen decreases with age, it was suggested by Verzar (20) that increased molecular crosslinking of collagen explains the age-related increase in TBT and the noted effects on collagen solubility. Ever since, the exact chemical structures of these putative markers of senescence have been sought (25). One such marker is a glycoxidation product, pentosidine, originally isolated from collagen of aged humans (26). Pentosidine has been found also in other mammals and in broiler breeder hens (13, 27-29). Pentosidine is an imidazopyridinium crosslink involving a pentose sugar crosslinked with arginine and lysine residues (26). 
Class aves is an interesting model for biogerontology (30): concentrations of plasma glucose are typically 2 to 6 times mammalian norms (30-32), metabolic rates are as much as 2 to 2.5 times higher than similarly-sized mammals (33-34), and basal body temperature is about $3{ }^{\circ} \mathrm{C}$ higher than in mammals (30). All these factors should accelerate the formation of advanced Maillard products and hence, the process of tissue aging compared to normal aging (30, 35-37). Concentrations of pentosidine in skin of birds, however, are approximately 1000 fold lower than what is found in mammalian tissues (32).

The objective of this study was to compare the tendon breaking time (TBT) and tendon breaking strength (TBS) of the flexor perforans et perforatus digiti iii tendon in broiler breeder hens with concentrations of pentosidine in tendon $\left(\mathrm{P}_{t}\right)$ from 8 to 125 wks of age. Secondly, effects of diet restriction (DR) and crosslinking inhibitor, aminoguanidine (AG) on $\mathrm{P}_{t}$, TBT and TBS were determined.

\section{MATERIALS AND METHODS}

\section{Birds and Management}

Day-old broiler breeder (Cobb X Cobb) female chicks $(n=450)$ were placed in electrically heated battery brooders and fed ad libitum until 4 wks of age. At this time, chicks were randomly assigned to four treatment groups: ad libitum (AL); diet restricted (DR) fed at $60 \%$ of AL energy intake; AL with aminoguanidine (AG; Aldrich Chemical Co., Inc., Milwaukee, WI 53233 ) at $1.35 \mathrm{mg} / \mathrm{kg} \mathrm{BW} /$ day $(\mathrm{AL}+\mathrm{AG})$; and DR with $\mathrm{AG}$ at $60 \%$ of that fed the AL group $(\mathrm{DR}+\mathrm{AG}) . \mathrm{AG}$ was supplemented in feed of the hens. Birds were fed these diets throughout the study. The DR chicks received the recommended amounts of vitamins, minerals and nutrients according to the Cobb Management Guide (Cobb-Vantress, Inc., Siloam Springs, AR 72761). Feed and AG allowance for DR birds were calculated weekly based on the previous week's consumption by the AL group. At the selected dose of $A G$, a significant reduction in the accumulation of fluorescent endproducts occurred in Biceps femoris muscle of broiler breeder hens (38). All birds were fed daily between 0800 and $1000 \mathrm{hrs}$ and had free access to water. The flock was reared in floor pens until 20 wks of age. At this time, hens were caged 
individually. All birds were kept under light-tight conditions, and photoperiod was set according to the Cobb Management Guide.

Tendon Collection. Flexor perforans et perforatus digiti iii tendons were removed at 12 wk intervals from 8 to 92 wks and then again at 125 wks of age. This tendon is among the longest tendons, extending from the middle of the tibial region to the 3rd digit. Hens $(n=5$, except for the AL group at 125 wks where only 2 birds remained) from each group were randomly selected for tendon dissection. Tendons were removed by transverse cuts at the point of insertion and slightly proximal to the origin, washed with normal saline and stored at $-80^{\circ} \mathrm{C}$ until physical and chemical analyses.

Measurement of Tendon Breaking Time and Strength. Tendon breaking time (TBT) and tendon breaking strength (TBS ) was measured by a modification of the procedures described for the estimation of shear force in pectoralis major muscle in poultry (39). Prior to analysis each tendon was immersed in Ringer's solution, $\mathrm{pH}$ 7.4. Determination of TBT and TBS was on individual tendons mounted in an Instron Universal Mechanical Machine (Model TM, Instron Corp., Canton, MA 45419). The normal grip to grip length of each test specimen was $10 \mathrm{~mm}$ (40). Tendons were attached between the Instron's movable cross-head and rigid base such that its position remained centered between the jaws. The cross-head, attached to a 50 kg load cell (Model 152050; Daytronic, Miamisburg, OH 45342), moved away from the rigid base at a speed of $50.8 \mathrm{~mm} / \mathrm{min}$. Output from a LVDT conditioner (Model 9130, Daytronic Miamisburg, $\mathrm{OH} 45342$ ) for the tensile deformation of the tendon was acquired by a computer equipped with a DT 2805 data acquisition board (Data Translation, Marlboro, MA 01752). Signals were processed with the HP-VEE software package (Hewllet Packard Co., Love land, CO 80539-9929). The unloaded cross-sectional area at the mid-point of the tendon was used to normalize the TBS. Tendon breaking time was recorded when the movable cross-head started to lift up until the tendon was broken. Tendon breaking strength was expressed as peak force (pf) in $\mathrm{kg} / \mathrm{mm}^{2}$ and TBT as time in milliseconds (ms). 


\section{Tendon Pentosidine $\left(\mathbf{P}_{t}\right)$ Determination}

Preparation of Collagen Digest. Broken Flexor perforans et perforatus digiti iii tendons were used for collagen digest for pentosidine determination. The collagen digest was prepared according to the techniques described by Monnier et al. (5) and Sell et al. (41). Briefly, $20 \mathrm{mg}$ of tendon was frozen in liquid nitrogen, minced, and placed into a 13 X $100 \mathrm{~mm}$ screw-capped tube and then delipidated overnight in a chloroform-methanol (2:1) solution. Samples were rehydrated in a $50 \%$ methanol solution and hydrolyzed in $5 \mathrm{ml}$ deareated $6 \mathrm{M} \mathrm{HCl}$ at $110{ }^{\circ} \mathrm{C}$ for $18 \mathrm{~h}$. All tubes were flushed with nitrogen prior to sealing with Teflon-faced rubber-lined caps. Subsequent to the hydrolysis, the samples were placed into a Speed Vac centrifuge-type vacuum drier (Savant Instruments, Farmingdale, NY 11735) until the $\mathrm{HCl}$ was evaporated. Samples were reconstituted in $250 \mu 1 \mathrm{H}_{2} \mathrm{O}$ containing $0.01 \mathrm{ml}$ hepatafluorobutyric acid (HFBA) and filtered using a Costar ${ }^{\circledR}$ Spin- $X ®$ centrifuge tube filter (Corning Costar Corp., Cambridge, MA 02140). A modified Stegman and Stadler spectrophotometric method was used for estimation of collagen, using a hydroxyproline standard and assuming a collagen content of $14 \%$ hydroxyproline by weight (42).

Tendon Collagen Pentosidine. Tendon pentosidine $\left(\mathrm{P}_{t}\right)$ was measured by a modified reversed phase HPLC method (29). Samples of $\sim 50$ to $200 \mu \mathrm{l}$ volumes equivalent to $1 \mathrm{mg}$ of collagen were injected into a 0.46X25 cm, Vydac 218TP104 $(10 \mu \mathrm{m}) \mathrm{C}-18$ column (Vydac, Hesperia, CA 92345) connected to a Waters HPLC (Waters, Milford, MA 01757). The apparatus consisted of two pumps (Waters ${ }^{\mathrm{TM}} 600$ Controller), an auto sampler (Waters ${ }^{\mathrm{TM}} 717_{\text {plus }}$ ), and a scanning florescence detector (Waters ${ }^{\mathrm{TM}} 474_{\text {Plus }}$ ). Separations were achieved by a linear gradient of $12-42 \%$ acetonitrile from 0 to $25 \mathrm{~min}$ in water and 0.01M HFBA at flow rate of 1 $\mathrm{ml} / \mathrm{min}$. The pentosidine peak was monitored by an on line scanning fluorescence detector at excitation wavelength $325 \mathrm{~nm} /$ emission wavelength $370 \mathrm{~nm}$. Quantitation of pentosidine was made by comparison with standard curve made by peak areas with a pentosidine standard (Vincent M. Monnier, Cleveland, OH 44120) injected under identical conditions. A software package (Millennium 2.1) was used for integration of peaks. 


\section{Statistical Analyses}

Data were analyzed by the general linear models procedure using 2 X 2 factorial design. (43). Correlation between $P_{t}$, age, TBS and TBT were determined. The Student-Newman-Keuls Multiple Range test was used to estimate the significance of difference among means.

\section{RESULTS}

Pentosidine $\left(\mathrm{P}_{\mathrm{t}}\right)$ of AL-fed broiler breeder hens increased with increasing age $\left(r^{2}=0.79\right.$, $p<0.0001)$. Concurrently, there was an age-related parallel increase in TBS $\left(r^{2}=0.56\right.$, $p<0.0001)$, and in TBT $\left(r^{2}=0.67, p<0.0001\right)$. DR lowered $(p<0.001)$ the accumulation of $\mathrm{P}_{\mathrm{t}}$ (Fig. 1A). Similarly, DR retarded the overall TBS $(p<0.01)$ and TBT $(p<0.02)$ when compared to tendons from AL-fed hens (Fig. $1 \mathrm{~B}, \mathrm{C}$ ). The effect of DR was not consistent on these endpoints; a greater decrease (112\%) in the concentration of $\mathrm{P}_{t}$ was associated with a relatively lower decrease in both TBS (55\%) and TBT (33\%) respectively.

Supplementation of AL-fed hens with AG (AL+AG group) retarded $(p<0.0002) \mathrm{P}_{\mathrm{t}}$ accumulation and resulted in decreases in TBS and TBT (p<0.01) (Fig. 2). As with DR, a 130\% decrease in the concentration of $P_{t}$ was measured in AL hens fed AG (AL+AG group) and was associated with $48 \%$ and $35 \%$ decreases in TBS and TBT, respectively. Supplementation of DR hens with AG (DR+AG group) did not affect ( $\mathrm{p}>0.05) \mathrm{P}_{\mathrm{t}}$, TBS or TBT (Fig. 3).

\section{DISCUSSION}

In the present study, age-related changes in $\mathrm{P}_{\mathrm{t}}$, TBS and TBT in broiler breeder hens were compared with the effects of DR and AG supplementation on these end-points. A parallel age-related increase in the concentration of $\mathrm{P}_{\mathrm{t}}$ was associated with age-related increases in TBS and TBT in AL-fed hens. These observations are similar to those of Fu et al. (44-45) who found a parallel increase in pentosidine formation and collagen crosslinking when rat tendon tendons were incubated with glucose. Likewise, Richard et al. (46) showed a parallel increase in pentosidine and TBT when tail tendons were incubated with ribose. However, because the lag phase and the percent increase in pentosidine and TBT were not the same over the incubation 
period in that study, there appeared to be some dissociation between the two events. These observations suggest that other crosslinks or factors may be responsible for the large increase in TBT during this type of incubation protocol. Undoubtedly, pentosidine would contribute to the observed increase in TBT if estimates of Vater et al. (47) are correct (14). The relationship between TBT and collagen crosslinks was further confirmed by the fact that the overall pattern of changes in TBT shared similarities with those of another glycoxidation product, carboxymethyllysine (CML) (48). Numerous studies have shown TBT to be highly correlated with age $(20,24,49-50)$, whereas little information is available about TBS in the literature.

DR markedly affected age-related changes in $P_{t}$, TBT and TBS. In mammals, DR has been found to increase mean and maximum life span while delaying many age-associated disease processes such as tumorigenesis (51-52), leukemia (53), and renal diseases (22). Previous studies have shown that the physiological rate of collagen aging as determined by TBT is delayed by DR (1,21-22). Similar observations were found in the present study. A significant delay of the age-related increase in TBS and TBT was observed in DR hens as compared to AL-fed hens (Fig. 1). Since DR delays the aging process, this intervention would be expected to also retard the age-related increase in the concentration of pentosidine. The rate of formation of $\mathrm{P}_{t}$ was significantly delayed by DR and these findings are comparable to those of earlier studies in rat tail tendons $(14,54)$.

The effectiveness of AG as an inhibitor of nonenzymatic glycation is controversial (1, 38, 55-60). There is also disagreement over its mode of action (61). In the present study, accumulation of $\mathrm{P}_{\mathrm{t}}$ for the AL+AG group was retarded (Fig. 2A); whereas, AG had no effect in the DR+AG group (Fig. 3A). Supplementation of AL and DR hens with AG resulted in a reduction in $\mathrm{P}_{\mathrm{t}}$ of $130 \%$ and $18 \%$, respectively. The overall concentration of $\mathrm{P}_{\mathrm{t}}$, TBS and TBT for AL+AG group was comparable to those of DR-fed birds (Fig. 1,2). The reduction in concentration of $\mathrm{P}_{\mathrm{t}}(130 \%)$ for the $\mathrm{AL}+\mathrm{AG}$ group was associated with a $48 \%$ decrease in TBS and a 35\% decrease in TBT. In contrast to these observations, Oxlund and Andreassen (1), did not find any difference between TBT of tail tendons from diabetic rats treated with AG for 120 
$\mathrm{d}$ and control rats. Results suggest that the detection of treatment effect is a likely explanation for these differences because a difference in TBT was recorded in the present study beginning at $20 \mathrm{wk}(140 \mathrm{~d})$ of age. In agreement with Oxlund and Andreassen (1), AG supplementation of DR did not affect $P_{t}$, TBS and TBT. These findings are consistent with those of Klandorf $e t$ al. (38) who reported no effect of AG supplementation in DR birds on the fluorescence of Biceps femoris muscles in poultry. Similarly, in a companion study (39), supplementation of DR with AG did not affect the shear force of the pectoralis major muscles over a $125 \mathrm{wk}$ period. These results are consistent with the views that AG is relatively ineffective in DR-fed animals. Perhaps this is due to the total concentration of pentosidine in a given amount of collagen, and the extent of glycosylation in the body. Dyer et al. (62) reported that pentosidine accounts for $<1 \%$ of the crosslinks formed from the in vitro Browning reaction between protein and glucose. Reports mention that glycosylation does not continue indefinitely and is limited in amounts that range from $\sim 0.4$ to $20 \%$ by weight of covalently attached carbohydrates, depending on the collagen's tissue of origin (63-65). Although DR significant reduced crosslinking, it did not reduce the glycosylation (32). As a consequence, DR probably left little or no role to play for AG supplementation in reducing the glycosylation process and subsequently reduction in collagen crosslinks. Although the mode of action in lowering $\mathrm{P}_{\mathrm{t}}$, TBS and TBT in AL-fed hens is not clearly understood, AG lowers oxidative stress in broiler breeder hens (32). However, oxidative stress in that study has to be carefully interpreted because there were only two birds in the ALfed group, and that was based only on one time point (125 wks) observation in broiler breeder hens.

Although rate of accumulation of $\mathrm{P}_{\mathrm{t}}$ was similar to the age-related changes in TBS and TBT, there were differences in response of these end-points to DR and AG. A 130\% decrease in mean overall $\mathrm{P}_{\mathrm{t}}$ for $\mathrm{AL}$ group was reported by supplementing this group with $\mathrm{AG}$ which was associated with $48 \%$ and 35\% decrease in mean TBS and TBT values, respectively. Similarly, a $112 \%$ decrease in mean overall concentration of $P_{t}$ in DR birds, which was associated with 55\% and 33\% decreases in mean TBS and TBT values, respectively. These observations are in contrast to studies in mammals (44-46) which showed that TBT values were relatively higher as compared to the crosslinks present in rat tail tendons incubated with glucose or ribose, 
suggesting other crosslinks than pentosidine might be involved in observed TBT values $(14,47)$. A non-fluorescent component (NFC-1) has been isolated (66) and its concentration is also glucose dependent. NFC-1 concentrations are also found 50 times greater than that of pentosidine in aorta (67). We do not know the reasons for this discrepancy between these results and the results of our study. On the other hand, an $18 \%$ reduction in the concentration of $\mathrm{P}_{\mathrm{t}}$ in DR+AG birds was associated with no changes in TBS and TBT (Fig. 3). Variable effects of DR and AG have been reported in literature on glycosylation $(13,32,68)$. The results from our earlier studies (32) and other previous work do not support the view of increased crosslink formation as a result of hyperglycemia per se. We have previously observed that DR and AG lowered the crosslinking without any observable effect on plasma glucose concentrations. These findings are intriguing and imply that glycosylation may not be the primary cause of age-related crosslink formation (45,69-71). In support of this view, there are reports that Amadori products are not likely to be the major precursors of collagen crosslinks and that crosslinking agents are generated, in part, before Amadori rearrangement (72).

In summary, $\mathrm{P}_{\mathrm{t}}$, TBS and TBT increased over time in AL-fed hens. DR significantly retarded the rate of accumulation of $P_{t}$, TBS and TBT alone, not with AG supplementation. In conclusion, AG was effective only in AL and not in DR hens. The response of DR and AG was greater in lowering the concentration of $\mathrm{P}_{t}$ than those of TBS and TBT. The results support the view that it is not hyperglycemia per se that is responsible for the age-related changes in tissue crosslinking.

Acknowledgments: The authors would like to thank Drs. John Killefer for his useful discussion in developing the method for TBS and TBT, and Edwin C. Townsend for his help with the statistical analyses. We are also grateful to Hakan Kocamis for his generous help in the accomplishment of this project. 


\section{REFERENCES}

1. Oxlund, H., and Andreassen, T.T. Aminoguanidine treatment reduces the increase in collagen stability of rats with experimental diabetes mellitus. Diabetologia 1992; 35:1925.

2. Kohn R.R., Cerami, A., and Monnier, V.M. Collagen aging in vitro by nonenzymatic glycosylation and browning. Diabetes 1984; 33:57-59.

3. Kent M.J.C., Light, N.D., and Bailey, A.J. Evidence for glucose-mediated covalent crosslinking of collagen after glycosylation in vitro. Biochem J 1985; 225-: 545-52.

4. Brownlee, M., Cerami, A., and Vlassara, H. Advanced products of nonenzymatic glycosylation and the pathogenesis of diabetic vascular disease. Diabetes Metabolism Reviews 1988; 4:437-451.

5. Monnier, V.M., Vishwanath, V., Frank, K.E., Elmets, C.A., Dauchot, P. and Kohn, P. R. Relationship between complications of type I diabetes mellitus and collagen-linked fluorescence. N. Engl. J. Med. 1986; 314:403-408.

6. Monnier, V.M. Nonenzymatic glycosylation, the Maillard reaction and the aging process. J. Gerontol: Biol. Sci. 1990; 45:B105-B111.

7. Reiser, K.M. Nonenzymatic glycation of collagen in aging and diabetes. Proc. Soc. Exp. Biol. Med. 1991; 196:17-29.

8. Bucala, R., and Cerami, A. Advanced glycosylation: chemistry, biology, and implications for diabetes and aging. Adv Pharmacol 1992; 23:1-33.

9. Kohn, R.R. Evidence against cellular aging theories. In: Adelman, R.C.: Roth, G.S., eds. Testing the theories of aging. Boca Raton, FL: CRC Press, 1982; 221-231.

10. Higgins, K.A., Stout, J.T., Heller, D.A., and Parker, R.F. Individual variability in tail tendon fiber break time in three age cohorts of different strains of mice. Exp. Gerontol. 1991; 26:467- 477.

11. Heller, D.A., and McClearn, G.E. A longitudinal genetic study of tail tendon fiber break time. Ageing 1992; 21:129-134. 
12. Reiser, K.M. Influence of age and long-term dietary restriction on enzymatically mediated crosslinks and nonenzymatic glycation of collagen in mice. J. Gerontol: Biol. Sci. 1994; 49:B71-B79.

13. Cefalu, W.T., Bell-Farrow, A.D., Wang, Z.Q., Sonntag, W.E., Fu, M., Baynes, J.W., and Thorpe, S.R. Caloric restriction decreases age-dependent accumulation of the glycoxidation products, $\mathrm{N}$-(Carboxymethyl)lysine and pentosidine, in rat skin collagen. J. Gerontol: Biol. Sci. 1995; 50A:B337-B341.

14. Sell, R. and Monnier, V.M. Age-related association of tail tendon break time with tissue pentosidine in DBA/2 vs C57BL/6 mice: The effect of dietary restriction. J. Gerontol: Biol. Sci. 1997;52A:B277-B284.

15. Andreassen T.T., and Oxlund, J. Thermal stability of collagen in relation to nonenzymatic glycosylation and browning in vitro. Diabetologia 1985; 28:687-69.

16. Andreassen, T.T., Oxlund, J., and Danielsen, C.C. The influence of nonenzymatic glycosylation and formation of fluorescent reaction products on the mechanical properties of rat tail tendons. Conn Tissue Res. 1988; 17:1-9.

17. Menzel, E.J., and Reihsner, R. Alterations of biochemical and biomechanical properties of rat tail tendon caused by nonenzymatic glycation and their inhibition by dibasic amino acid arginine and lysine. Diabtologia 1991, 34:12-16.

18. Galeski, A., Kastelic, J., Baer, E., and Kohn, R.R. Mechanical and structural changes in rat tail tendon induced by alloxan diabetes and aging. J Biomech. 1977; 10:775-782.

19. Andreassen T.T., Seyer-Hansen, K., and Bailey, A.J. Thermal stability, mechanical properties and reducible crosslinks of rat tail tendon in experimental diabetes. Bichem. Biophys. Acta 1981; 677:313-317.

20. Verzar, F. The aging of collagen. Sci. Am. 1963; 208:104-114.

21. Everitt, A.V., Porter, B.D., and Steele, M. Dietary, caging and temperature factors in the aging of collagen fibers in rat tail tendon. Gerontology 1981; 27:37-41.

22. Everitt, A.V., Wyndham, J.R., and Barnare, D.L. The anti-aging action of hypophysectomy in hypothalamic obese rats: effects on collagen aging, age-associated proteinuria development and renal histopathology. Mech. Aging Dev. 1983; 22:233-25. 
23. Harrison, D.E., and Archer, J. R. Physiological assays for biological age in mice: Relationship of collagen, renal function, and longevity. Exp. Aging Res. 1983; 9:245251.

24. Harrison, D.E., Archer, J.R., Sacher, G.A., Boyce, F.M. Tail collagen aging in mice of thirteen different genotypes and two species: Relationship to biological age. Exp. Gerontol. 1978; 13:63-73.

25. Calkins, E. Aging of cells and people. Clin. Obstet. Gynecol. 1981; 24:165-179.

26. Sell, D.R., and Monnier, V.M. Structural elucidation of senescence crosslink from human extra-cellular matrix: Implication of pentoses in the aging process. J. Biol. Chem. 1989; 264:21597-21602.

27. Grandhee, S.K., and Monnier, V.M. Mechanisms of formation of the Maillard protein crosslink pentosidine: ribose, glucose, fructose and ascorbate as pentosidine precursors. J. Biol. Chem. 1991; 266:11649-53.

28. Dyer, D.G., Blackledge, J.A., Thorpe, J.R., and Baynes, F.W. Formation of pentosidine during nonenzymatic browning of proteins by glucose: Identification of glucose and other carbohydrates as possible precursors of pentosidine in vivo. J. Biol. Chem. 1991b; 266:11654-11660

29. Iqbal, M., Probert, L.L., and Klandorf, H. Effect of aminoguanidine on tissue pentosidine and reproductive performance in broiler breeders. Poultry Sci. 1997; 76:1574-1579.

30. Holmes, J.D., and. Austad, S.N. Birds as animal models for the comparative Biology: A prospectus. J. Geront: Biol. Sci. 1995; 50A:B59-B66.

31. Beuchat, C.A., and Chong, C.R. Hyperglycemia in hummingbirds: Implications for hummingbird ecology and human health. FASEB J. 1997; 11:3:A91.

32. Iqbal, M., and Probert, L.L., Klandorf, H. and Al-Humadi, N.H. Protein glycosylation and accumulation of advanced glycosylation endproducts (AGEs): An avian Solution? J Gerontology: Biol. Sci. 1998; In review.

33. Lindstedt, S., and Calde, W. Body size and longevity in birds. Condor 1976; 78:91-94.

34. Austad, S., and Fischer, K. Mammalian aging, metabolism, and ecology: evidence from bats and marsupials. J. Gerontol: Biol. Sci. 1991;46:B47-B53. 
35. Harman, D. Aging: A theory based on free radical and radiation chemistry. J. Gerontol: Biol. Sci. 1956;11:289-300.

36. Del Maestro, R. An approach to free radicals in medicine and biology. Acta Physiol. Scand. 1980;492:153-168.

37. Sohal, R., and Allen, R. Oxidative stress as a causal factor in differentiations and aging: A unifying hypothesis. Exp. Gerontol. 1990; 25:499-522.

38. Klandorf, H., Zhou, Q., and Sams, A.R. Inhibition by aminoguanidine of glucose-derived collagen cross-linking in skeletal muscle of broiler breeder hens. Poultry Sci. 1996; 75:432-437.

39. Iqbal, M., Kenney, P.B., and Klandorf, H. Age-related changes in meat tenderness and tissue pentosidine: Effect of diet restriction and aminoguanidine in broiler breeder hens. Poultry Sci.1998; In review.

40. Haut, R.C. The influence of specimen length on the tensile failure properties of tendon collagen. J. Biomechanics 1986; 19:951-955.

41. Sell, D.R., Lapolla, A., Odetti, P., Fogarty, J., and Monnier, V.M. Pentosidine formation in skin correlates with severity of complications in individuals with long-standing IDDM. Diabetes. 1992; 41:1286-1291.

42. Maekawa, T., Ratinasamy, Y.K, Altman, K.I., and Forbes, W.F. Changes in collagen with age-I. The extraction of acid soluble collagens from skin of mice. Exp. Gerontol. 1970; 5:177-186.

43. SAS Institute. SAT/STAT User's Guide: Statistics: Release 6.04 SAS Institute Inc., Cary, NC. 1990.

44. Fu, M.-X., Knecht, K. J., Thrope, S.R., and Baynes, J. W. Role of oxygen in crosslinking and chemical modification of collagen by glucose. Diabetes 1992; 41 (Suppl. 2):42-48.

45. Fu, M.-X., Wells-Knecht, K.J., Blackledge, J. A., Lyons, T. J., Thrope, S.R., and Baynes, J.W. Glycation, glycoxidation, and crosslinking of collagen by glucose. Kinetics, mechanisms, and inhibition of late stages of the Maillard reaction. Diabetes 1994; 43:676-683. 
46. Richard, S., Tamas, C., Sell, D.R., and Monnier, V.M. Tissue-specific effects of aldose reductase inhibition on fluorescence and crosslinking of extracellular matrix in chronic galactosemia. Relationship to pentosidine crosslinks. Diabetes 1991; 40:1049-1056.

47. Vater, C.A., Harris, Jr., E.D., and Siegel, R.C. Native crosslinks in collagen fibrils induce resistance to human synovial collagenase. Biochem. J. 1979; 181:639-645.

48. Elgawish, A., Glomb, M., Friedlander, M. and Monnier, V.M. Involvement of hydrogen peroxide in collagen crosslinking by high glucose in vitro and in vivo. J. Biol. Chem. 1996; 271:12964-12971.

49. Everitt, A.V. Food intake, growth and the aging of collagen in rat tail tendon. Gerontologia 1971; 17:98-108.

50. Harrison, D.E., and Archer, J. R. Measurement of changes in mouse tail collagen with age: Temperature dependence and procedural details. Exp. Gerontol. 1978;13:75-82

51. Weindruch, R., and Walford, R.L. Dietary restriction in mice beginning at 1 year of age: Effect of life-span and spontaneous cancer incidence. Science 1982; 215a;1415-1418.

52. Snyder, D.L., ed. Dietary restriction and aging: Proceedings of the symposium on the effects of dietary restriction of aging and disease in germfree and conventional LobundWistar rats. Proc. Clin. Biol. Res. 1989;289 New York: Alan R. Liss.

53. Shimokawa, I., Yu, B.P., Higani, Y., Ikeda, T., and Masoro, E.J. Dietary restriction retards onset but not progression of leukemia in male F344 rats. 1993. J. Gerontol: Biol. Sci. 48:868-B73.

54. Sell, D.R., Lane, M.A., Johnson, W.A., Masoro, E.J., Mock, O.B., Reiser, K.M., Fogarty, J.F., Cutler, R.G., Ingram, D.K., Roth, G.S., and Monnier, V.M. Longevity and the genetic determination of collagen glycoxidation kinetics in mammalian senescence. Proc. Natl. Acad. Sci. 1996; 93:485-490.

55. Brownlee, M., Cerami, A. and Vlassara, H. Advanced products of nonenzymatic glycosylation and the pathogenesis of diabetic vascular disease. Diabetes Metabolism Reviews.1988; 4:437-451. 
56. Khatan, M., Soldan, Z., David, I., Li, W. and Rockey, J.H. Inhibitory effects of pyridoxal phosphate, ascorbate and aminoguanidine on nonenzymatic glycosylation. Life Sci. 1988; 43:1725-1731.

57. Odetti, P.R., Borgoglio, A., Pascale, A.D., Polandi, R. and Adezati, L. Prevention of diabetes-increased aging effect on rat collagen-linked fluorescence by aminoguanidine and rutin. Diabetes 1990; 39:796-801.

58. Mathieu, C., Waer, M., Casteels, K., Laureys, J., and Bouillon, R. Prevention of Type I diabetes in NOD mice by non-hypercalcemic doses of a new structural analog of 1, 25dihydroxyvitamin $\mathrm{D}_{3}$, DH 1060. Endocrinology 1995; 136:866-872.

59. Kochakian, M., Manjula, B.N., and Egan, J.J. Chronic dosing with aminoguanidine and novel advanced glycosylation endproduct-formation inhibitors ameliorates crosslinking of tail tendon collagen in STZ-induced diabetic rats. Diabetes 1998; 45:1694-1996.

60. Crijns, R.L., Boudier, H.A.J.S., Wolfenbuttel, B.H.R. Arteriolar reactivity in conscious diabetic rats: Influence of aminoguanidine treatment. Diabetes 1998; 47:918-923.

61. Booth, A.A., Khalifah, R.G., Todd, P. \& Hudson, B.G. In vitro kinetic studies of formation of antigenic advanced glycation end products (AGEs): Novel inhibition of post-Amadori glycation pathways. J. Biol. Chem. 1997; 272, 5430-5437.

62. Dyer, D.G., Blackledge, J.A., Thorpe, J.A., and Baynes, F.W. Formation of pentosidine during nonenzymatic browning of proteins by glucose: Identification of glucose and other carbohydrates as possible precursors of pentosidine in vivo. J. Biol. Chem. 1991b; 266:11654-6.

63. Eyre, D.R. Collagen: Molecular diversity in the body's protein scaffold. Science 1980; 207:1315-1327.

64. Sims, T.J., and Bailey, A. J. The structure and growth of muscle. Pages 27-47 in: Meat Science. R.A. Lawrie. ed. 5th, New York.1982.

65. Bailey, A.J., and Light, N.D. The structure and growth of muscle. Pages 27-47 in: Meat Science. R. A. Lawrie. ed. 5th, New York.1989.

66. Sims, T.J., and Bailey, A.J. Quantitative analysis of collagen and elastin crosslinks using a single column system. J. Chromatog 1992; 582: 49-55. 
67. Sims, T.J., Rasmussen, L.M., Oxlund, H., and Bailey, A.J. The role of glycation crosslinks in diabetic vascular stiffening. Diabetologia 1996; 39: 946-951.

68. Masoro, E., Katz, M., and McMahan, C. Evidence for the glycation hypothesis of aging from the food-restricted rodent model. J. Gerontol: Biol. Sci. 1989; 44:B20-B22.

69. Guitton, J.D., Le Pape, A., and Muh, J.P. Influences of in vitro nonenzymatic glycosylation on the physicochemical parameters of Type I collagen. Collagen Relat. Res. 1984; 4:253-264.

70. Le Pape, A., Guitton, J.D., and Muh, J.P. Distribution of non-enzymatically bound glucose in vivo and in vitro glycosylated Type I collagen molecules. FEBS Lett. 1984;170:23-27.

71. Lyons, T.J., Bailie, K.E., Dyer, D.G., Dunn, J.A., and Baynes, J.W. Decrease in skin collagen glycation with improved glycemic control in patients with insulin-dependent diabetes mellitus. J. Clin. Invest. 1991;87:1910-1915.

72. Monnier, V., Glomb, M., Elgawish, A., and Sell, D. The mechanism of collagen crosslinking in diabetes: A puzzle nearing resolution. Diabetes 1996; 45:S67-S72. 
Figure 1. Effect of age and diet restriction (DR) on (A) concentration of pentosidine $\left(\mathrm{P}_{\mathrm{t}}\right),(\mathrm{B})$ tendon breaking strength (TBS), (C) tendon breaking time (TBT) in flexor perforans et perforatus digiti iii tendon of broiler breeder hens. Each point represents the mean ( $\mathrm{n}=5$; except at $125 \mathrm{wks}(\mathrm{n}=2)$ for AL group) \pm SEM. Differences were significant, ${ }^{*} p<0.05$, $* * p<0.001$ and $* * * p<0.0001$, between AL (closed squares) and DR (open circles) groups at those points.

Figure 2. Effect of age and aminoguanidine (AG) supplementation with ad libitum-fed (AL) on (A) concentration of pentosidine $\left(\mathrm{P}_{\mathrm{t}}\right)$; (B) tendon breaking strength (TBS); (C) tendon breaking time (TBT) in flexor perforans et perforatus digiti iii tendon of broiler breeder hens. Each point represents the mean $(n=5$; except at 125 wks $(n=2)$ for AL group) \pm SEM. Differences were significant, ${ }^{*} p<0.05, * * p<0.001$ and $* * * p<0.0001$ between AL (closed squares) and AL+AG (open triangles) groups at those points.

Figure 3. Effect of age, diet restriction (DR) and aminoguanidine (AG) on (A) concentration of pentosidine $\left(\mathrm{P}_{\mathrm{t}}\right)$; (B) tendon breaking strength (TBS); (C) tendon breaking time (TBT) in flexor perforans et perforatus digiti iii tendon of broiler breeder hens. Each point represents the mean $(\mathrm{n}=5) \pm$ SEM. Supplementation of DR with AG did not affect these end-points significantly ( $p>0.05)$ between DR (closed diamonds) and DR+AG (open circles) groups. 
Figure 1

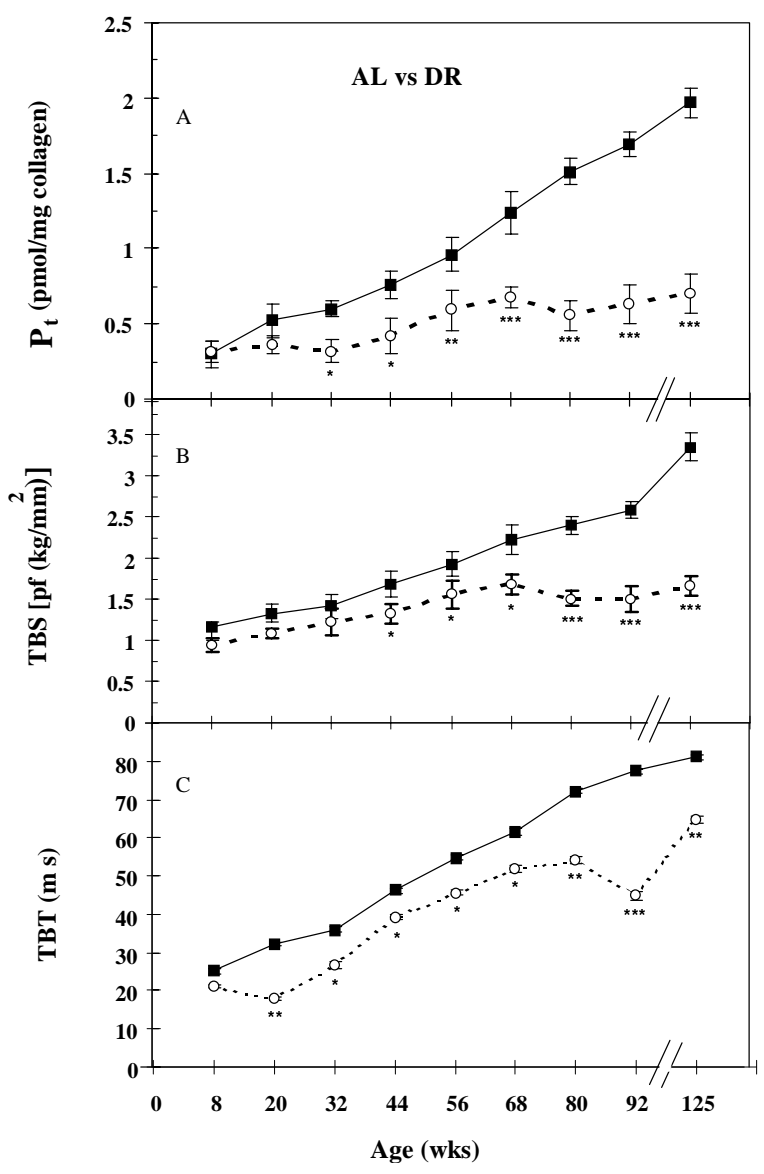




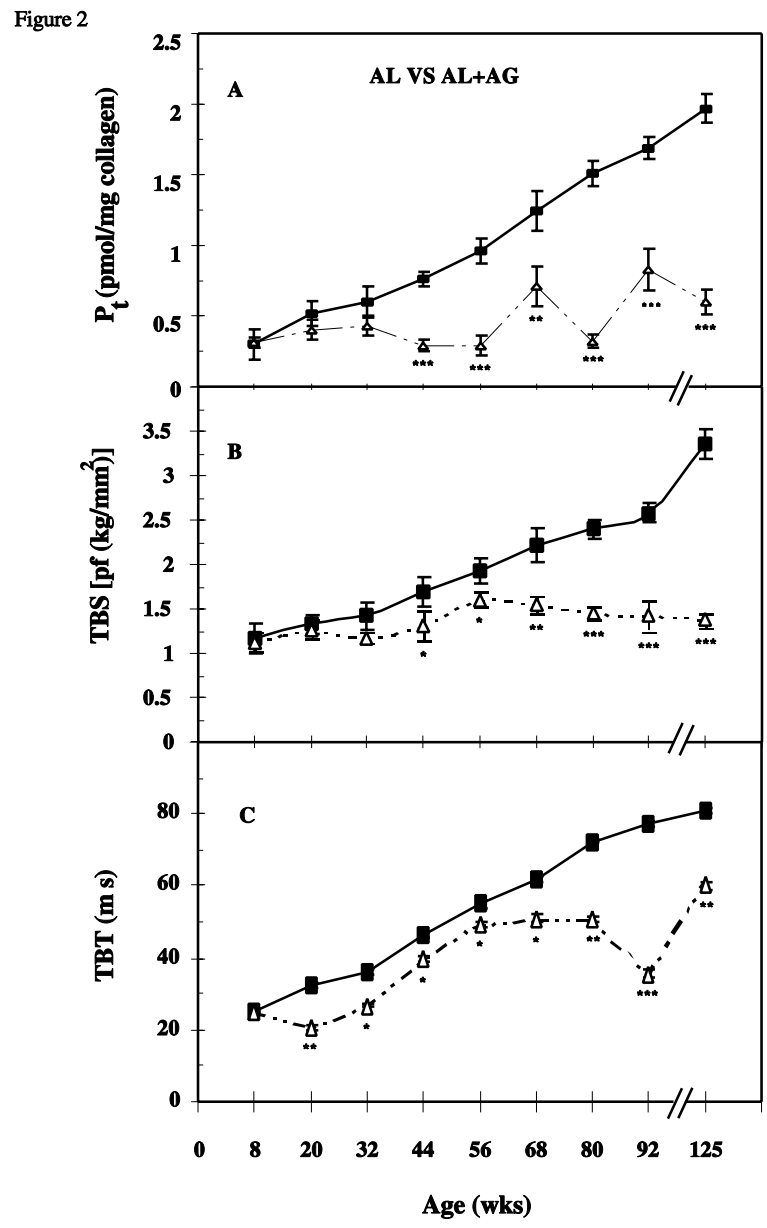




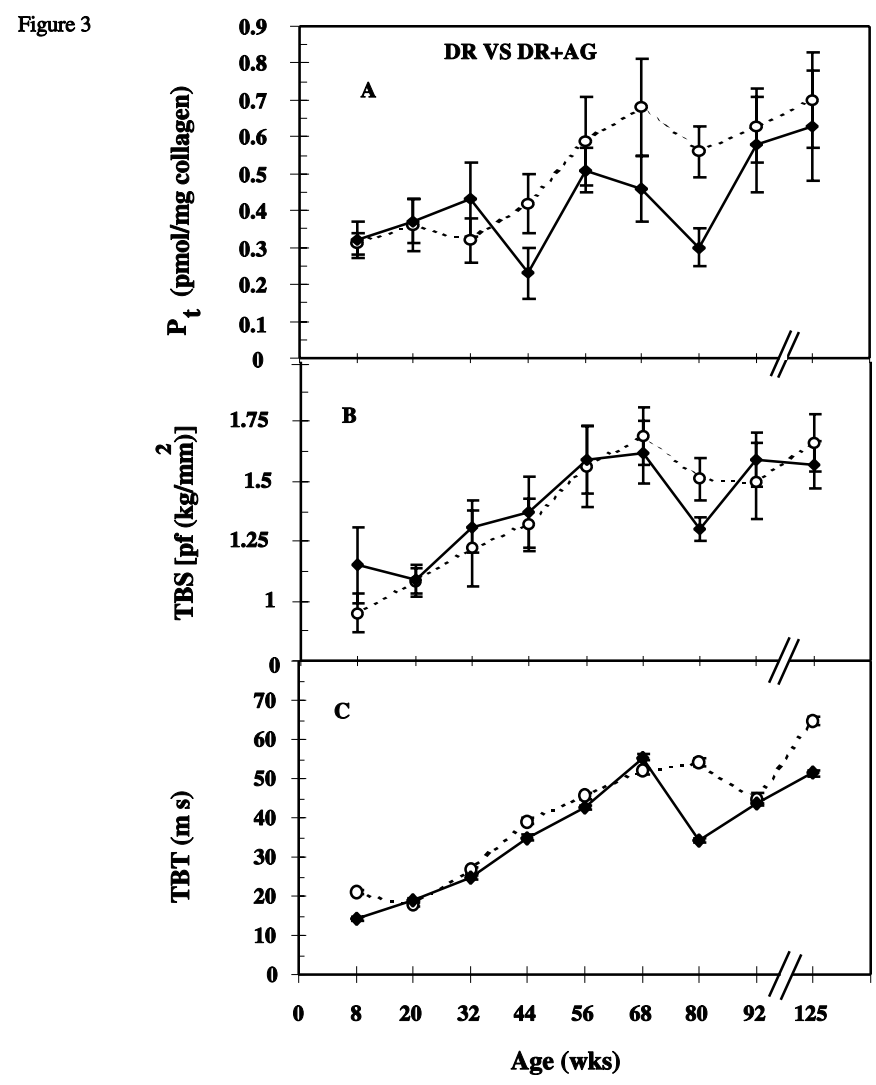




\title{
Study III
}

\section{Age-related Changes in Meat Tenderness and Tissue Pentosidine: Effect of Diet Restriction and Aminoguanidine in Broiler Breeder Hens}

\begin{abstract}
The nonenzymatic glycosylation of tissue protein contributes to the formation of crosslinks that leads to structural and functional deterioration in the long-lived tissue protein, collagen. The accumulation of these crosslinks thus contributes to the objectionable toughness of meat from aged animals, decreases its economic value and limits its use in whole muscle foods. The objectives of this study were to determine the effectiveness of diet restriction (DR) and the crosslinking inhibitor, aminoguanidine (AG), on reducing the accumulation of crosslinks, thereby improving meat tenderness in broiler breeder hens. The glycoxidation product, pentosidine, was also measured in skin (Ps) to determine if changes in its concentrations correlated with the changes in shear force $(\mathrm{SF})$. Chicks $(n=450)$ were randomly assigned to four treatment groups from 8 to 125 wks after hatch: ad libitum (AL); diet restricted (DR); ad libitum and diet restricted groups supplemented with $400 \mathrm{ppm}$ AG each (AL+AG and DR+AG respectively). SF was measured with an Instron Universal Mechanical Machine. Ps was isolated by reverse phase HPLC. There was an age-related, linear increase in SF $(p<0.0001, r=0.96)$ which correlated $(r=0.86)$ with the age-related increase in Ps in AL fed hens. DR significantly retarded the SF $(p<0.0001)$ over the sampling period. In general, SF values for AL+AG were similar to those measured in DR; whereas, no additive effect was observed for AG in DR birds. It was concluded that there was a linear increase in meat toughness (SF) with age that correlates with the accumulation of Ps, and that the decline in meat tenderness can be retarded by DR or
\end{abstract}


AG. Secondly, the effect of DR on accumulation of Ps was so pronounced that AG supplementation did not further enhance this effect.

Key words: Diet restriction, Aminoguanidine, Meat tenderness, Pentosidine, Aging.

\section{INTRODUCTION}

Meat of spent fowls becomes objectionably tough with increasing age and this toughness limits its use in whole muscle foods resulting in considerable economic losses to the poultry industry (Nakamura et al., 1975; Shimokomaki et al., 1972). For this reasons meat has been traditionally used in less-profitable, comminuted or retorted products where the small particle size or thermal processing is used to reduce toughness (Nurmahmudi and Sams, 1997). This problem is of particular importance to the poultry industry which produces spent fowl as a by-product of table and hatching egg production. Approximately 130,000,000 spent fowl being disposed of in the USA annually (Vandepopuliere and Lyons 1996); thus, the potential economic benefit to be gained by tenderizing this meat is tremendous.

The age-related decline in meat tenderness is due to many factors. Recent studies have suggested that glucose-derived, nonenzymatic collagen crosslinking plays a role in the normal aging process as well as the accelerated tissue aging associated with the hyperglycemic state (Brownlee et al., 1988). The structural, long-lived tissue protein collagen is the major component of connective tissue and functions as the basic supporting structure of tissues and organs. Nonenzymatic collagen crosslinking in muscle may contribute to the decline in meat tenderness of aged animals; although, its importance in skeletal muscle has not been established (Bailey, 1989; Klandorf et al., 1996). The glycosylation theory of aging suggests that modification of proteins by glucose and associated browning or Maillard reactions lead to the gradual crosslinking, polymerization, development of brown color and fluorescence characteristic of age-associated changes in tissue protein collagen (Cerami, 1985; Monnier, 1989). Accumulation of these crosslinks are postulated to lead to structural and functional deterioration of tissue protein and contribute to the pathophysiology of diabetic complications (Monnier, 1989; Bucala and Cerami, 1992; Vlassara et al., 1994; Cefalu et al., 1995). One such 
crosslink, pentosidine, has been isolated and characterized in numerous animal species (Sell and Monnier, 1989; Iqbal et al., 1997), and is an imidazole [4, 5b] pyridinium molecule containing a lysine and an arginine residue crosslinked by a pentose.

Presently, there is no economically feasible way to increase meat tenderness by decreasing collagen crosslinking in spent fowl meat (Kondaiah and Panda, 1992; Woods et al., 1997). Numerous studies have demonstrated that diet restriction (DR) (Masoro et al., 1989; Cefalu et al., 1995) and the crosslinking inhibitor, aminoguanidine (AG) (Brownlee et al., 1986; Khatan et al., 1988; Oxlund and Andrassen, 1992; Wu, 1995), retard the accumulation of crosslinks over an animal's life time. Whether DR, AG or both (Klandorf et al., 1996; Iqbal et al., 1997), can improve meat tenderness (SF) as compared to ad libitum fed birds has not been determined. In addition, the glycoxidation product, pentosidine, was measured in skin (Ps) to determine if changes in its concentration correlated with internal changes in shear force (SF) values of the pectoralis major muscle.

\section{MATERIALS AND METHODS}

\section{Birds and Management}

Day-old broiler breeder (Cobb X Cobb) female $(n=450)$ chicks were placed in electrically heated battery brooders and fed ad libitum until 4 wks of age. At this time, chicks were randomly assigned to four treatment groups: ad libitum (AL group); diet restricted (DR group); ad libitum with 400 ppm aminoguanidine ${ }^{1}$ (AG); and diet restricted groups with 400 ppm AG in feed ( $\mathrm{AL}+\mathrm{AG}$ and $\mathrm{DR}+\mathrm{AG}$ groups respectively). Birds were fed accordingly throughout the study. Diet restricted chicks were fed daily with a limited allowance diet according to the Cobb Management Guide ${ }^{2}$. At the selected dose of AG, a significant reduction in the accumulation of fluorescent endproducts occurred in B. femoris muscle of broiler breeder hens (Klandorf et al., 1996). All birds were fed daily between 0800 and $1000 \mathrm{hrs}$ and had free access to water.

\footnotetext{
${ }^{1}$ Aldrich Chemical Co., Inc., Milwaukee, WI 53233.

${ }^{2}$ Cobb-Vantress, Inc., Siloam Springs, AR 72761.
} 
The flock was reared in floor pens until 20 wks of age. At this time, hens were caged individually. All birds were kept under light tight conditions, and photoperiod was set according to the Cobb Management Guide. Body weight was monitored weekly.

\section{Meat Tenderness Evaluation}

Breast muscle tenderness was determined with modification of the methods described by Kenney et al., (1996).

Breast Muscle Collection. Tissue samples were obtained at 12 wk intervals from 8 to 92 wks and then again at 125 wks of age. Hens $(n=5)$ from each group were randomly selected for slaughter. Birds were electrically stunned and bled using a modified kosher technique. The pectoralis major muscle was isolated after $4 \mathrm{~h}$ of refrigeration at $4 \mathrm{C}$, vacuum packed, and stored at $-20 \mathrm{C}$ until thermal processing.

Measurement of Shear Force. The pectoralis major muscle from each bird was cooked to an internal temperature of $70 \mathrm{C}$ on a Farberware Smokeless Indoor Grill ${ }^{3}$. Endpoint internal temperature (CT) was monitored with an Industrial Datalogger ${ }^{4}$ equipped with a copper-constant thermocouple ${ }^{5}$. Cooked muscle was cooled to room temperature and refrigerated overnight at $4 \mathrm{C}$. Slices of $1.27 \mathrm{~cm}$ were cut perpendicular to the fiber orientation of the muscle. Four to six, $1.27 \mathrm{~cm}$ diameter cores were removed from these slices parallel to the fiber orientation through the thickest portion of the cooked muscle. Warner-Bratzler shear force (SF) was determined by using an Instron Universal Mechanical Machine ${ }^{6}$. A Warner-Bratzler apparatus was attached to

\footnotetext{
${ }^{3}$ Model 450N, Farberware, Inc., Bronx, NY 10462.

${ }^{4}$ Model 205, Beckman Instruments, San Diego, CA 92123-1898.

${ }^{5}$ Omega Technologies; Stamford, CT 06907.

${ }^{6}$ Model TM, Instron Corp., Canton, MA 45419.
} 
a $50 \mathrm{~kg} \mathrm{load} \mathrm{cell}{ }^{7}$ and tests were performed at a cross head speed of $127 \mathrm{~mm} / \mathrm{min}$. Output from a LVDT conditioner ${ }^{8}$ was acquired by a computer equipped with a DT 2805 data acquisition board $^{9}$. Signals were processed with the HP-VEE software package ${ }^{10}$.

Breast Weight and Cook Yield. Raw and cooked breast weights (Br. W) were recorded. Cook yield (CY) was calculated by expressing cooked weight as a \% of uncooked weight.

\section{Pentosidine Determination}

Preparation of Collagen Digest. Hens $(n=5)$ from the AL group at all time points were randomly selected and sacrificed as described earlier. Approximately $1 \mathrm{~g}$ of skin was removed from the breast area, washed with normal saline and stored at $-80 \mathrm{C}$ until assayed. The collagen digest used for pentosidine determination was prepared according to the techniques described by Monnier et al. (1986) and Sell et al. (1992). Briefly, this involved the removal of epidermal and adipose layers from skin samples, freezing in liquid nitrogen, and mincing. The minced samples were delipidated overnight in a chloroform-methanol (2:1) solution. Samples were rehydrated in a $50 \%$ methanol solution and hydrolyzed in $6 \mathrm{~N} \mathrm{HCl}$ at $110 \mathrm{C}$ for $18 \mathrm{~h}$. All tubes were flushed with $\mathrm{N}_{2}$ prior to capping for heating. Subsequent to the hydrolysis, the samples were placed into a Speed Vac centrifuge-type vacuum drier ${ }^{11}$ until the $\mathrm{HCl}$ was evaporated. Samples were reconstituted in $250 \mu \mathrm{L} \mathrm{H}_{2} \mathrm{O}$ and filtered using a Costar ${ }^{\circledR}$ Spin-X® centrifuge tube filter $^{12}$. A modified Stegman and Stadler spectrophotometric method was used for collagen estimation, using a hydroxyproline standard (Maekawa et al., 1970).

\footnotetext{
${ }^{7}$ Model 152050; Daytronic, Miamisburg, OH 45342.

${ }^{8}$ Model 9130, Daytronic Miamisburg, OH 45342.

${ }^{9}$ Data Translation, Marlboro, MA 01752.

${ }^{10}$ Hewllet packard Co., Love land, CO 80539-9929.

${ }^{11}$ Savant Instruments, Farmingdale, NY 11735.

${ }^{12}$ Corning Costar Corp., Cambridge, MA 02140.
} 
Skin Collagen Pentosidine. Age-related changes in skin pentosidine (Ps) from 8 to 92 wks of age were determined in a separate study (Iqbal et al., 1998). For purposes of establishing a relationship with shear force in this study, tissue pentosidine is reported in AL-fed birds only from 8 to 125 wks of age. Estimation of pentosidine was done by a modified reversed phase HPLC method (Iqbal et al., 1997). One mg of acid-hydrolyzed collagen digest in $100 \mu \mathrm{L}$ of water/0.01M heptafluorobutyric acid (HFBA) was injected into a 0.46X25 cm Vydac 218TP104 $(10 \mu \mathrm{m}) \mathrm{C}-18$ column $^{13}$ connected to a Shimadzu HPLC ${ }^{14}$. The apparatus consisted of two model LC-600 pumps, a model SIL-6B auto injector, a model RF 551 fluorescence monitor (excitation $325 \mathrm{~nm}$, emission $370 \mathrm{~nm}$ ). Separations were achieved by application of a linear gradient of 12$42 \%$ acetonitrile from 0 to $20 \mathrm{~min}$ in water and $0.01 \mathrm{M}$ HFBA. Quantitation of pentosidine was made by comparison of peak areas with a pentosidine standard ${ }^{15}$ injected under identical conditions. A software package (Shimadzu CLASS-VP 4.2) was used to analyze the data.

\section{Statistical Analyses}

Data were analyzed by the general linear models procedure using a $2 \times 2 \times$ time factorial design (SAS Institute, 1990). Regression analysis was carried out to determine the correlation between SF and Ps. Covariate analyses were carried out with age, CT and Br. W as covariates of SF in order to eliminate their potential effects on treatments. The Student-Newman-Keuls Range method was used to test the significance of difference between means.

\section{RESULTS AND DISCUSSION}

In the present study, the potential contribution of nonenzymatic crosslinks to meat tenderness has been addressed in broiler breeder hens over a 125 wks period. In both skin and

\footnotetext{
${ }^{13}$ Vydac, Hesperia, CA 92345.

${ }^{14}$ Shimadzu, Inc., Columbia, MD 21040.

${ }^{15}$ Vincent M. Monnier, Cleveland, $\mathrm{OH} 44120$.
} 
muscle Type I and III collagen are the two predominant genetic types of collagen (Sims and Bailey, 1982; Bailey and Light, 1989) and so a specific tissue crosslink, Ps, was used as an indicator of glycative and oxidative tissue damage in these tissues (Baynes, 1991). In mammals, pentosidine has been found to increase over the life span of the individual (Grandhee and Monnier, 1991; Dyer et al., 1991b; Cefalu et al., 1995). In agreement with these studies, there was an age-related, linear increase in Ps $(p<0.001, r=0.95)$ that correlated $(r=0.86)$ with the linear increase in SF of pectoralis major muscle in AL-fed hens (Fig. 1). Nakamura et al., (1975) reported that SF values of pectoralis major muscle increased with age $(r=0.955)$ in different strains of White Leghorn chicken up to 520 days of age. Similar observations were also reported by Awonorin and Ayoade (1992) in broilers; Robertson et al., (1984) in buffalo and BrahmanSouthorn steers; and Batcher et al., (1962) on lamb cuts from different ages.

Because crosslinks play an important role in the normal aging process and diabetes, the utility of DR and a pharmacological agent (AG) to attenuate the formation of these crosslinks was investigated. Various methods have been used to degrade the intrinsic collagen network to improve the texture of meat from older animals. These approaches include: acid marinades (Arganosa and Marriott, 1989); injection of plant proteinases such as papain (Brooks et al., 1985; Cronlund and Woychik, 1987); $\mathrm{Ca}^{+2}$ ion marinades and postmortem injections (Young and Lyon, 1989; Young et al., 1989; Papa et al., 1989; Koohmaraie et al., 1988; 1989; 1990); postmortem injection of bacterial collagenases into muscle (Foegeding and Larick, 1986; Cronlund and Woychik, 1987); activation of postmortem collagen proteolysis by the action of neutral proteases (Koohmaraie et al., 1987; Morgan et al., 1991; Birkhold et al., 1992; Nurmahmudi and Sams, 1997). However, most of these studies did not address changes in collagen and meat quality in older animals. DR is one of the most extensively used anti-aging measures (Weindrich and Walford, 1988; Synder, 1989) and has been reported to extend the life span in rodents (Berg and Simms, 1960; Yu et al., 1982a), reduce glycosylation (Masoro et al., 1989; Cefalu et al., 1995; Beuchat and Chong, 1997) and delay the onset of a number of major age-associated diseases (Yu, 1994b). In our study DR decreased SF values of pectoralis major muscle: the effect became particularly pronounced $(\mathrm{p}<0.0001)$ after 56 wks (Fig. 2). The mechanism whereby DR lowers 
the SF values is not clearly understood. However, a decrease in the level of oxidative stress and glycation in DR animals might have been limited the formation of glycoxidation products by increasing the concentration of antioxidant enzymes (Yu et al., 1982a; Youngman et al., 1992; $\mathrm{Yu}, 1994 \mathrm{~b})$, thus indirectly limiting the age-related decline in meat tenderness in birds.

Morgan et al., (1991) reported no differences in the thermal stability of collagen (total, heat-soluble, or heat-insoluble collagen) for $\mathrm{CaCl}_{2}$-injected vs control muscles obtained from mature cows. However, in young animals, Birkhold et al., (1992) reported increased meat tenderness due to structural degradation of the collagen network induced by calpains. However, these workers could not confirm that the proteolytic and tenderization were related. Measurement of calpain activity as a possible mechanism of action of DR in meat tenderness should be addressed in the future studies. Supplementation of AL with AG (AL+AG group) reduced ( $p<0.0001)$ SF values, however, no further decrease in $S F$ values was recorded ( $p>0.05$ ) for DR supplemented with AG (DR+AG group) (Fig. 2). This finding is consistent with a previous study in broiler breeder hens in which SF values of $B$. femoris muscle were not affected in DR birds supplemented with AG (Klandorf et al., 1996). It appears that AG is relatively ineffective in DR animals. This could be due to the fact that glycosylation does not continue infinitively and is limited in amounts that range from $\sim 0.4$ to $20 \%$ by weight of covalently attached carbohydrates, depending on the collagen's tissue of origin (Eyre, 1980; Sims and Bailey, 1982; Bailey and Light, 1989). Furthermore, pentosidine accounts for $<1 \%$ of the crosslinks formed during in vitro browning reaction of protein with glucose (Dyer et al., 1991). DR probably left little or no role to play for AG supplementation in reducing the glycosylation process and subsequently reduction in the meat toughness. Although the mode of action of AG in lowering SF values in the AL group is not clearly understood, AG has been found to lower oxidative stress (Iqbal et al., 1998) in broiler breeder hens. Whether its mode of action is comparable to that of DR has yet to be established.

There was also a linear, age-related increase in $\mathrm{Br}$. W ( $<0.0001, r=0.64)$ and $\mathrm{CT}$ $(\mathrm{p}<0.0001, r=0.95)$ in AL fed hens. These observations are consistent with those of Awonorin 
and Ayoade (1992) in broilers and turkeys, and Toumy and Lechnir (1964) in beef. DR significantly reduced $\mathrm{Br}$. W and CT $(\mathrm{p}<0.0001)$. Covariate analyses did not eliminate the treatment effects when Br. W, CT and age were kept as covariates of SF. In general, there was no consistent effect of AG supplementation on breast weight and CT, however, a marked decrease in CT was observed at 80 wks in the AL+AG group (Fig.3, 4). No consistent effect of DR and AG was observed on cook yield (data not reported).

In summary, increased meat toughness (SF) with age correlates with accumulation of Ps, in AL-fed animals. The decline in meat tenderness can be retarded by DR or supplementation with AG. Furthermore, the effect of DR on crosslink accumulation is so pronounced that AG supplementation cannot further enhance this effect. The results of this study have potential implications, particularly for the use of skin as an indicator of age-related changes in muscle. Using this approach a skin sample can be used for pentosidine estimation and hence a predicator of meat tenderness. Secondly, the data support the use of AG in the diets of AL animals as to improve meat tenderness. Future studies could address pentosidine accumulation in specific muscles and relate these changes to SF values.

\section{Acknowledgments}

The authors wish to thank Nabil H. Al-humadi, NIOSH/HELD, Morgantown WV 26505, for his assistance in the HPLC analysis, Edwin C. Townsend, Agriculture and Forestry experimental station, West Virginia University, Morgantown, WV 26506, for his help in statistical analyses, and Hakan Kocamis and Lorree L. Probert, Division of Animal and Veterinary Sciences, West Virginia University, Morgantown, WV 26506, for technical assistance. 


\section{REFERENCES}

Arganosa, G. C., and N. G. Marriott, 1989. Organic acids as tenderizers of collagen in restructured beef. J. Food Sci. 54:1173-1175.

Awonorin, S. O. and J. A. Ayoade, 1992. Texture and eating quality of raw- and thawed- roasted turkey and chicken breasts as influenced by age of birds and period of frozen storage. J. Foodserv. Syst. 6:241-255.

Bailey, A. J. 1989. The chemistry of collagen crosslinks and their role in meat texture. Proc. Reciprocal Meat Conference. 42:127-135.

Bailey, A. J., and N. D. Light, 1989. The structure and growth of muscle. Pages 27-47 in: Meat Science. R. A. Lawrie. ed. 5th, New York.

Batcher, O. M., E. H. Dawson, M. T. Pointer, and G. L. Gilpin, 1962. Quality of raw and cooked lamb meat as related to fatness and age of animal. Food Technol. 16:102-110.

Baynes, J. W. 1991. Role of oxidative stress in development of complications in diabetes. Diabetes 40:405-412.

Berg, B. N., and H. S. Simms, 1960. Nutrition and longevity in the rat. II. Longevity and onset of disease with different levels of food intake. J. Nutr. 71:255-259.

Beuchat, C. A., and C. R. Chong, 1997. Hyperglycemia in hummingbirds: Implications for hummingbird ecology and human health. FASEB J.11:3:A91.

Birkhold, S. G., J. B. Morgan, R. K. Miller, and A. R. Sams, 1992. Proteolytic mechanism of mature cow meat tenderization by calcium injection. Page 153 in: Proceedings of Institute of 
Food Technologists Annual Meeting, New Orleans, LA.

Brooks, B. A., K. C. Klasing, and J. M. Regenstein, 1985. Effects of antemortem injected crude papain in chicken muscle. J. Food Sci. 50:1370-1374.

Brownlee, M., A. Cerami, and H. Vlassara, 1988. Advanced products of nonenzymatic glycosylation and the pathogenesis of diabetic vascular disease. Diabetes Metabolism Reviews. 437-451.

Brownlee, M., H. Vlassara, A. Kooney, P. Ulrich, A. Cerami, 1986. Aminoguanidine prevents diabetes-induced arterial wall protein cross-linking. Sci. 232:1629-1632.

Bucala, R., and A. Cerami, 1992. Advanced glycosylation: chemistry, biology, and implications for diabetes and aging. Adv. Pharmacol. 23:1-34.

Cefalu, W. T., A. D. Bell-Farrow, Z. Q. Wang, W. E. M. Sonntag, W. E. Fu, J. W. Baynes, and S. R. Thorpe, 1995. Caloric restriction decreases age-dependent accumulation of the glycoxidation products, $\mathrm{N}-($ Carboxymethyl) lysine and pentosidine, in rat skin collagen. J. Gerontol: Biol. Sci. 50A:B337-B341.

Cerami, A. 1985. Hypothesis: glucose as a mediator of aging. J. Am. Geriatr. Soc. 33:626-634. Cronlund, A. C. And J. H. Woychik, 1987. Solubilization of collagen in restructured beef with collagenases and alpha-amylase. J. Food Sci.52:857-860.

Dyer, D. G., J. A. Blackledge, J. A. Thorpe and F. W. Baynes, 1991b. Formation of pentosidine during nonenzymatic browning of proteins by glucose: identification of glucose and other carbohydrates as possible precursors of pentosidine in vivo. J. Biol. Chem. 266:11654-6. 
Eyre, D. R. 1980. Collagen: molecular diversity in the body's protein scaffold. Science 207:1315-1327.

Foegeding, E. A., and D. K. Larick, 1986. Tenderization of beef with bacterial collagenases. Meat Sci. 18:201-214.

Grandhee, S. K., and V. M. Monnier, 1991. Mechanisms of formation of the Maillard protein crosslink pentosidine: ribose, glucose, fructose and ascorbate as pentosidine precursors. J. Biol. Chem. 266:11649-53.

Iqbal, M., L. L. Probert, and H. Klandorf, 1997. Effect of dietary aminoguanidine on tissue pentosidine and reproductive performance in broiler breeder hens. Poultry Sci. 76:1574-1579.

Iqbal, M., L. L. Probert, N. H. Al-humadi and H. Klandorf, 1998a. Protein glycosylation and advanced glycosylation endproducts (AGE's): an avian solution. J. Gerontol:Biol. Sci. In review.

Kenney, P. B., S. D. Slider, R. R. Nayak, and J. W. Massey, 1996. Stunning method and time held in a transport coop affect $\mathrm{pH}$ decline and muscle quality in broilers. Poultry Sci.75: (Suppl.1-72):55.

Khatan, M., Z. Soldan, I. David, W. Li, and J. H. Rockey, 1988. Inhibitory effects of pyridoxal phosphate, ascorbate and aminoguanidine on nonenzymatic glycosylation. Life Sci. 43:17251731.

Klandorf, H., Q. Zhou, and A. R. Sams, 1996. Inhibition by aminoguanidine of glucose-derived collagen crosslinking in skeletal muscle of broiler breeder hens. Poultry Sci. 75:432-437. Kondaiah, N., and B. Panda, 1992. Processing and utilization of spent hens. World's Poult. Sci. 56:255-265. 
Koohmaraie, M., A. S. Babiker, A. L. Schroeder, R. A. Merkel, and T. R. Dutson, 1988. Acceleration of postmortem tenderization in ovine carcasses through activation of $\mathrm{Ca}^{+2}$ dependent proteases. J. Food Sci. 53:1638-1641.

Koohmaraie, M., J. D. Crouse, and J. J. Mersman, 1989. Acceleration of postmortem tenderization in ovine carcasses through infusion of calcium chloride: effect of concentration and ionic strength. J. Anim. Sci.67:934-942.

Koohmaraie, M., S. C. Seideman, J. E. Schollmeyer, T. R. Dutson, and J. D. Crouse, 1987. Effect of post-mortem storage on $\mathrm{Ca}^{+2}$-dependent proteases, their inhibitor and myofibril fragmentation. Meat Sci. 19:187-196.

Koohmarie, M., G. Whipple, and J. D. Crouse, 1990. Acceleration of postmortem tenderization in lamb and Barham-cross beef carcasses through infusion of calcium chloride. J. Anim. Sci. 68:1278-1283.

Maekawa, T., Y. K. Ratinasamy, K. I. Altman and W. F. Forbes. 1970. Changes in collagen with age-I. The extraction of acid soluble collagens from skin of mice. Exp. Geront. 5:177-186.

Masoro, E. J., M. S. Katz, and C. A. McMahan, 1989. Evidence for the glycation hypothesis of aging from the food-restricted rodent model. J. Gerontol: Biol. Sci. 44:B20-B22. Monnier, V. M. 1989. Toward a Maillard reaction theory of aging . Prog. Clin. Biol. Res. $304: 1-22$.

Monnier, V. M., V. Vishwanath, K. E. Frank, C. A. Elmets, P. Dauchot and P. R. Kohn. 1986. Relationship between complications of type I diabetes mellitus and collagen-linked fluorescence. N. Engl. J. Med. 314:403-408. 
Morgan, J., R. K. Miller, F. M. Mendez, D. S. Hale, and J. W. Savell, 1991. Using calcium chloride injection to improve tenderness of beef from mature cows. J. Anim. Sci. 69:4469-4476.

Nakamura, R., S. Sekoguchi, Y. Sato, 1975. The contribution of intramuscular collagen to the tenderness of meat from chickens with different ages. Poultry Sci. 54:1604-1612.

Nurmahmudi and A. R. Sams, 1997. Tenderizing spent fowl meat with calcium chloride. 1. Effect of delivery method and tumbling. Poultry Sci. 76:534-537.

Odetti, P. R., A. Borgoglio, A. D. Pascale, R. Polandi and L. Adezati, 1990. Prevention of diabetes-increased aging effect on rat collagen-linked fluorescence by aminoguanidine and rutin. Diabetes. 39:796-801.

Oxuland, H. T., And T. Andreassen, 1992. Aminoguanidine treatment reduces increase in collagen stability of rats with experimental diabetes mellitus. Diabetologia 35:19-25.

Papa, C. M., L. L. Young, and C. E. Lyon, 1989. Calcuim-induced tenderization of earlydeboned breast fillets. Poultry Sci. 68:110.

Robertson, J., D. Ratcliff, P. E. Bouton, P. V. Harris, and W. R. Shorthose, 1984. Effect of cooking temperature and animal age on the shear properties of beef and buffalo meat. J. Food Sci. 49:1163-1166.

SAS Institute, 1990. SAT/STAT ${ }^{\circledR}$ User's Guide: Statistics. Release 6.04 SAS Institute Inc., Cary, $\mathrm{NC}$.

Sell, D. R., A. Lapolla, P. Odetti, J. Fogarty, and V. M. Monnier, 1992. Pentosidine formation in skin correlates with severity of complications in individuals with long-standing IDDM. Diabetes, 41:1286-1291. 
Sell, D. R. and V. M. Monnier, 1989. Structure elucidation of a senescence crosslink from human extracellular matrix: Implication of pentoses in the aging process. J. Biol. Chem. 264:21597-21602.

Shimokomaki, M., D. F. Elsden, and A. J. Bailey, 1972. Meat tenderness: age-related changes in bovine intramuscular collagen. J. Food Sci. 37:892.

Sims, T. J., And A. J. Bailey, 1982. The structure and growth of muscle. Pages 27-47 in: Meat Science. R. A. Lawrie. ed. 5th, New York.

Synder, D. L., 1989. Dietary restriction and aging. in: Proceedings of the Symposium on the effects of dietary restriction of aging and disease in Germfree and conventional lobund-Wistar rats. Prog. Clin. Biol. Res. 289. New York.

Toumy, J. M., and R. J. Lechnir, 1964. Effect of cooking temperatures and time on the tenderness of beef. Food Technol. 17: 119-124.

Vandepopuliere and J. J. Lyons. 1996. Overview of spent hen alternatives. Proc. National poultry waste management. PP 113-118.

Vlassara, H., R. Bucala, L. Striker, 1994. Pathogenic effects of advanced glycosylation: biochemical, biologic, and clinical implications for diabetes and aging. Lab. Invest. 70: 138-151.

Weindruch, R., R. L. Walford, 1988. The retardation of aging and disease by dietary restriction. Springfield, IL"Charles C Thomas.

Woods, K. L., K. S. Rhee, and A. R. Sams, 1997. Tenderizing spent fowl meat with calcium chloride. 4. Improved oxidative stability and the effects of additional aging. Poultry Sci. 76:548551. 
Wu, G. 1995. Nitric oxide synthesis and effect of aminoguanidine and N-monomethyl-L-arginine on the onset of diabetes in spontaneously diabetic BB rats. Diabetes, 44:364-365.

Young, L. L., and C. E. Lyon, 1989. Effect of muscle $\mathrm{pH}$ and calcium content on quality of preand post-rigor chicken breast muscle. J. Food Sci. 54:1155-1157.

Young, L. L., and C. E. Lyon, H. Kim, C. M. Papa, and M. F. Miller, 1989. Effect of varying calcium content on functional and textural properties of chicken breast muscle. Poultry Sci. $68: 212$.

Youngman, L. D., J. Y. K. Park, B. N. Ames, 1992. Protein oxidation associated with aging is reduced by dietary restriction of protein or calories. Proc. Natl., Acad. Sci. 89:9112-9116. Yu, B. P. 1994b. How diet influences the aging process of the rat. Proc. Soc. Exp. Biol. Med. 205:97-107.

Yu, B. P., E. J. Masoro, I. Murafa, H. Q. Berfrand and F. T. Lynd, 1982a. Life-span study of SPF Fisher 344 male rats fed ad libitum or restricted diets: longevity, growth, lean body mass and disease. J. Gerontol.: Biol. Sci. 37:130. 
Figure 1. Correlation between shear force (SF) in pectoralis major muscle and pentosidine (Ps) in the skin ( $\mathrm{r}=0.86)$ of AL fed hens. Each point is the mean of 5 birds.

Figure 2. Effect of age, diet restriction (DR) and aminoguanidine (AG) on shear force (SF). Each bar represents mean $(n=5) \pm$ SEM. No common letters differ significantly $(\mathrm{p}<0.001)$ at 68 and 80 wks, and ( $<<0.0001)$ at 92 and 125 wks between AL and DR groups at those time points only. $\mathrm{pf}=$ peak force in $\mathrm{kg}$.

Figure 3. Effect of age, diet restriction (DR) and aminoguanidine (AG) on breast muscle weight (Br. W). Each bar is the mean $(\mathrm{n}=5) \pm$ SEM. No common letters differ significantly $(\mathrm{p}<0.0001)$ between AL and DR groups at all time points, and $(\mathrm{p}<0.05)$ in supplemented $(\mathrm{AL}+\mathrm{AG}$ and $\mathrm{DR}+\mathrm{AG})$ as compared to non-supplemented groups (AL and DR) at those time points only.

Figure 4. Effect of age, diet restriction (DR) and aminoguanidine (AG) on cook time (CT). Each bar is the mean $(n=5) \pm$ SEM. No common letters differ significantly $(\mathrm{p}<0.0001)$ between AL and DR groups at all time points, and $(\mathrm{p}<0.05)$ in supplemented $(\mathrm{AL}+\mathrm{AG}$ and $\mathrm{DR}+\mathrm{AG})$ as compared to non-supplemented groups (AL and DR) at those time points only. 
Figure 1

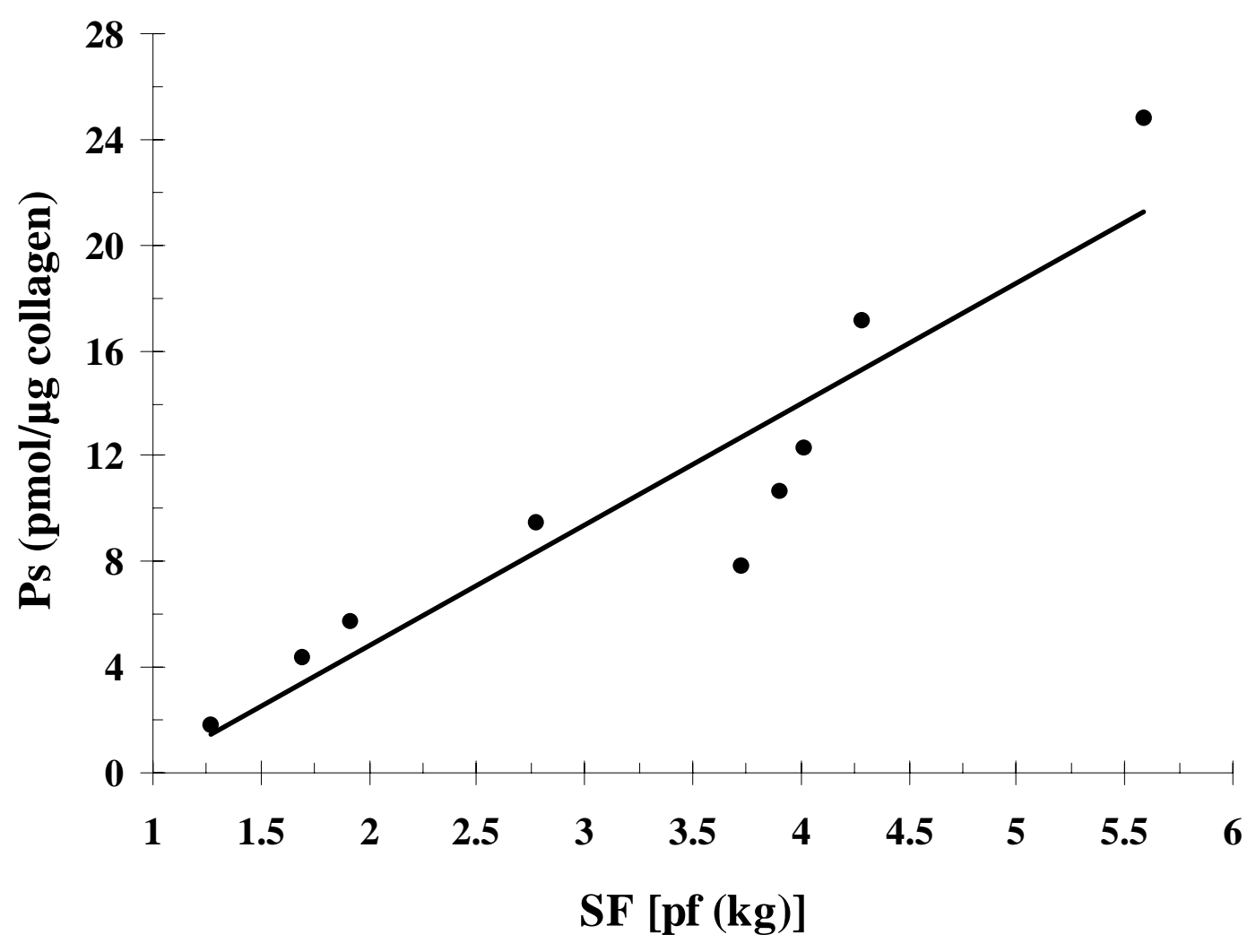


Figure 2

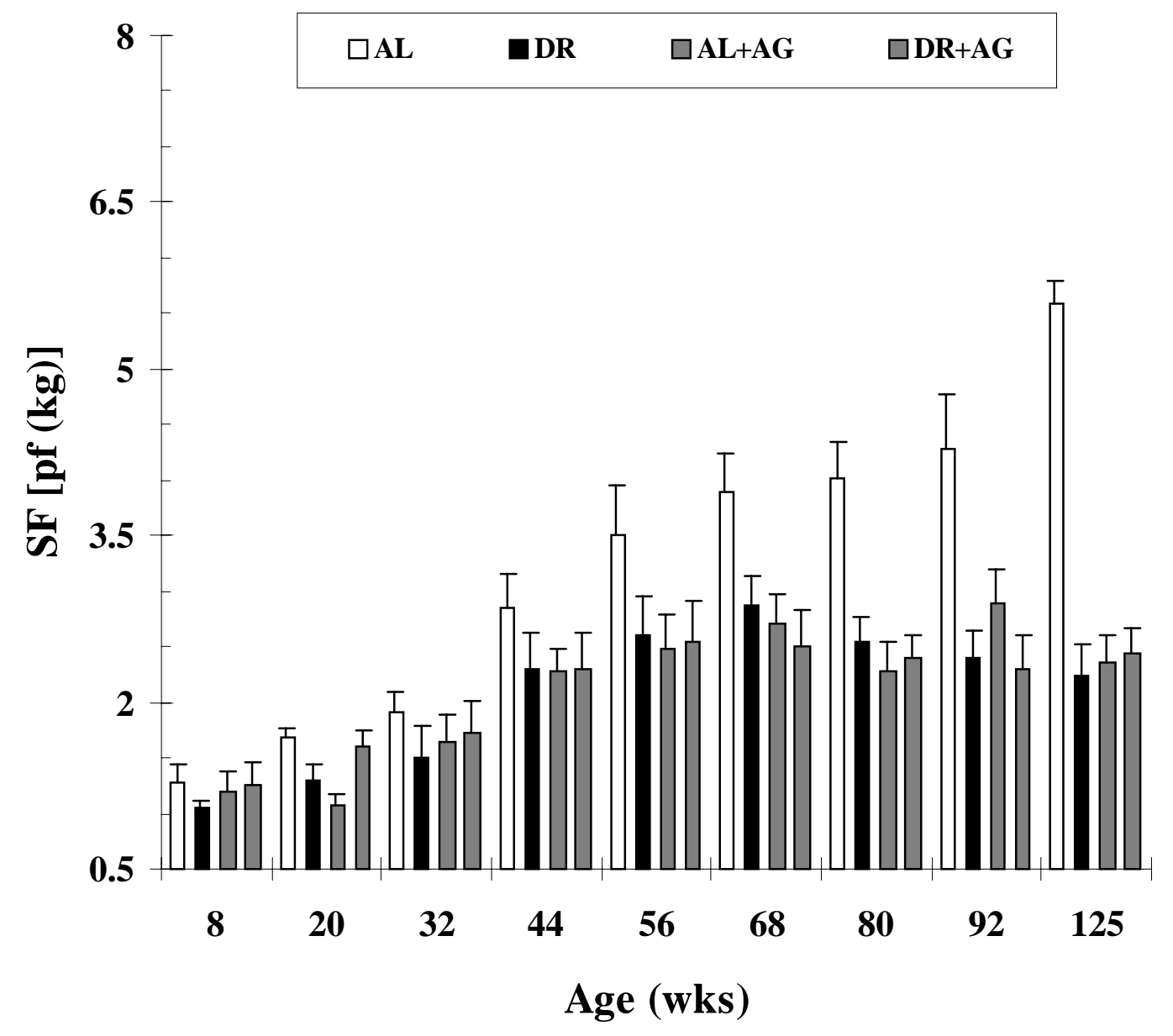




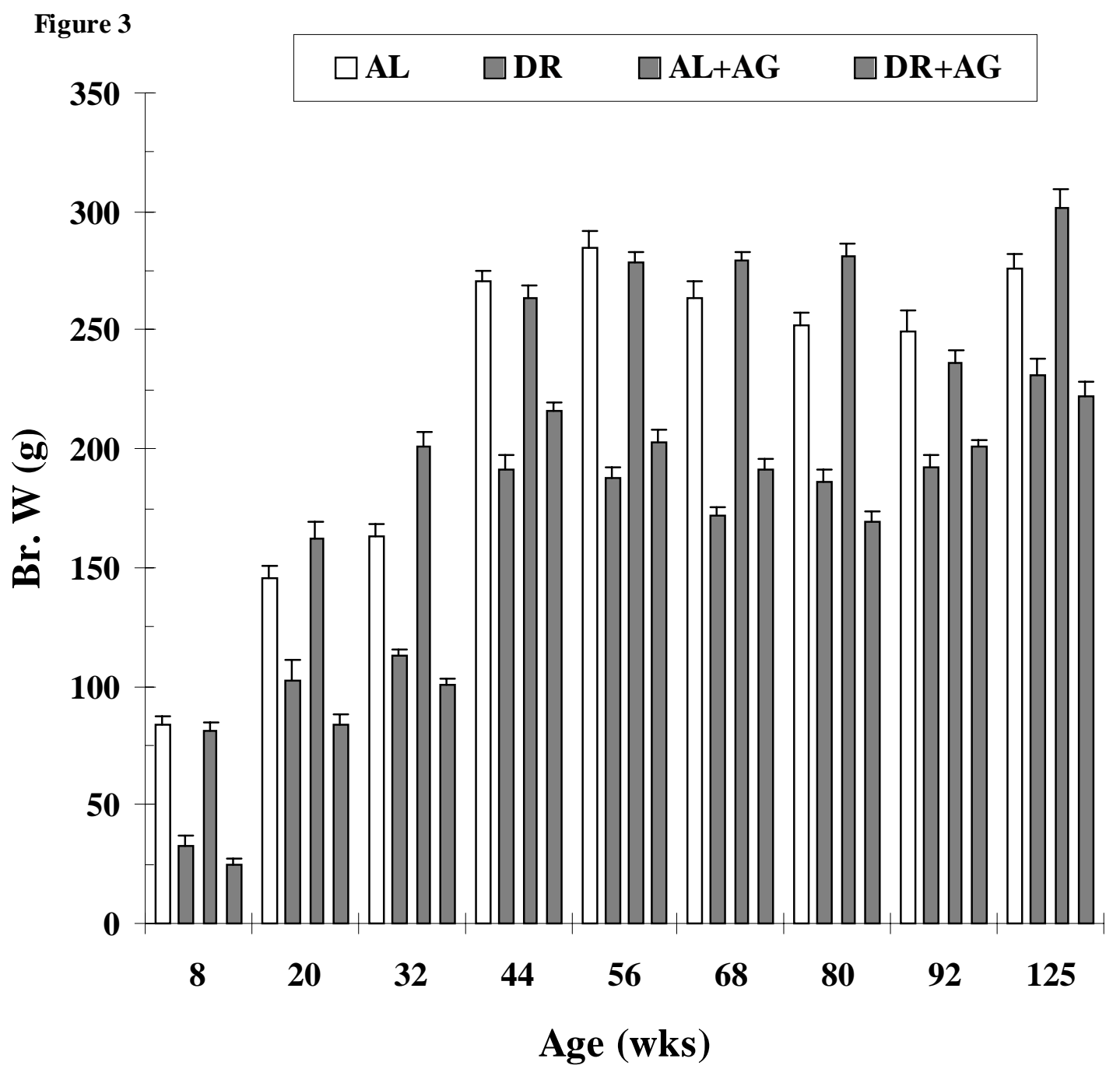


Figure 4

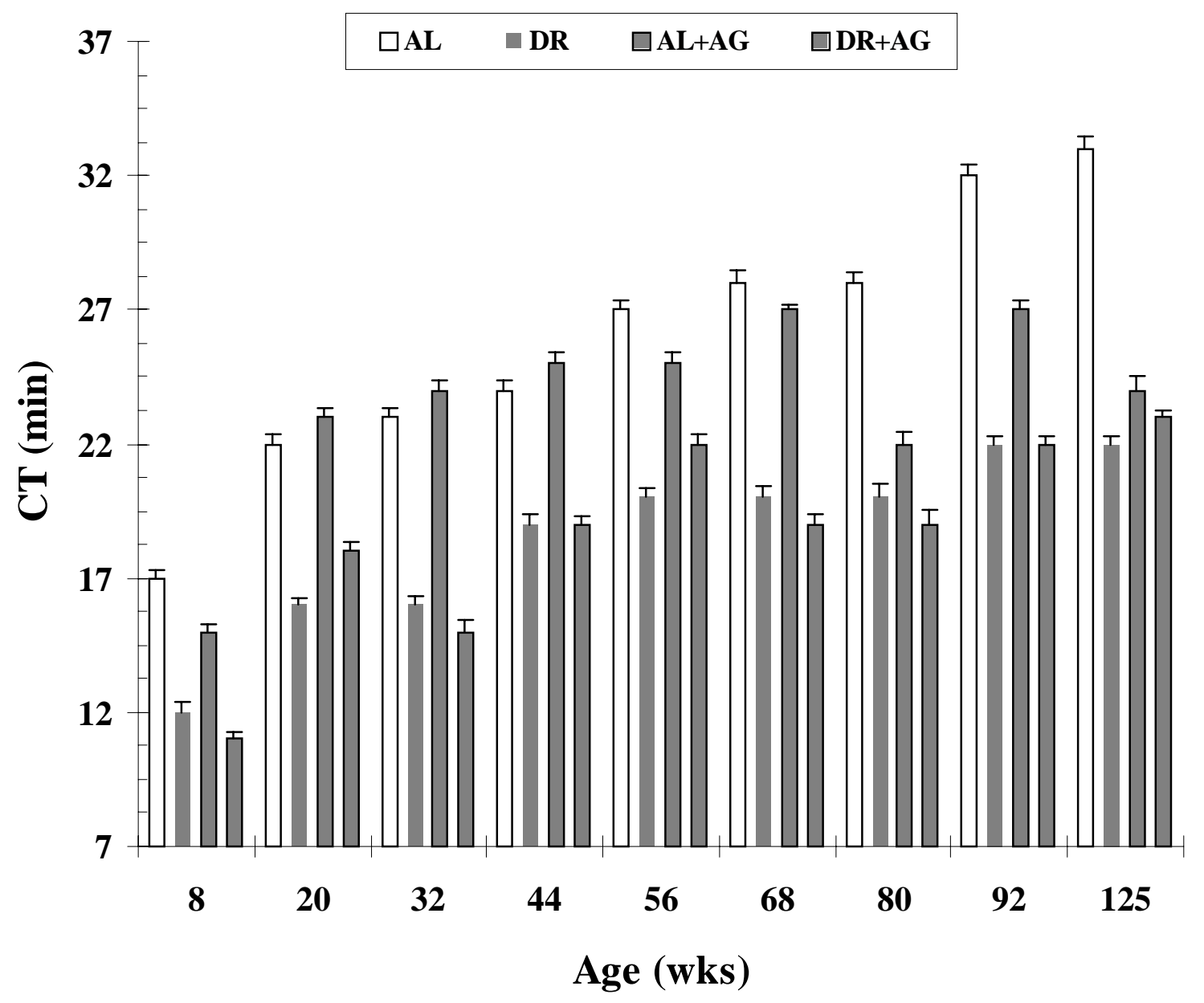




\title{
Study IV
}

\section{Effect of Dietary Aminoguanidine on Tissue Pentosidine and Reproductive Performance in Broiler Breeder Hens}

\begin{abstract}
Factors influencing the age-related decline in production parameters of broiler breeder hens are poorly understood. Elevated blood glucose concentrations measured in broiler breeder hens may contribute to this decline. The nonenzymatic attachment of glucose to proteins generates glycoxidation crosslinks in tissue proteins which can ultimately impair their function. One such glycoxidation crosslink, pentosidine, has been used as a biomarker for aging studies because of its accumulation on the structural protein collagen. The objectives of these studies were to determine if pentosidine accumulates with age in hens and if the crosslinking inhibitor, aminoguanidine (AG), could retard this accumulation. An additional objective was to determine if AG had any effect on production performance. In the first study broiler breeder hens $(n=318)$ were randomly assigned to two groups: control and supplemented (400 ppm AG). Pentosidine was measured in the skin of the birds at 20 and 67 wks of age. Egg production was measured daily. In a second study, broiler breeder hens $(n=60)$ were reared as previously described. Pentosidine was measured in the skin of the birds at 20 and 68 wks of age. Results showed that pentosidine was present in the skin of the hens, and that concentrations increased with age $(\mathrm{P}<0.001)$. Although pentosidine was reduced $(\mathrm{P}<0.001)$ in $\mathrm{AG}$ supplemented birds, production performance was not affected. In conclusion, AG retarded the rate of accumulation of pentosidine during lay in broiler breeder hens, but the reduction in pentosidine did not significantly affect production performance.
\end{abstract}

Key words: aging, diet restriction, aminoguanidine, pentosidine, broiler breeder 


\section{INTRODUCTION}

Advancing age adversely affects the reproductive success of many species including poultry. Once the genetic potential for maximum production of eggs has been reached, there is a steady, age-related decline in performance. Furthermore, eggs that are laid during the latter period of the reproductive lifespan are less likely to be fertilized and, if fertilized, are less likely to hatch (Lerner et al., 1993). The factors that influence these age-related decreases in reproductive success are poorly understood. However, there is evidence in male humans that adult onset diabetes accelerates the age-related decline in reproductive function and fertility (Kaiser and Korenman, 1988).

The reproductive lifespan of ad libitum fed broiler breeders is both shorter and less productive than their diet restricted counterparts; diet restriction (DR) is the only established means of delaying the decline in their productive performance. Extensive gerontological research within the last two decades has confirmed findings that restricting the food intake of laboratory animals extends their reproductive lifespan (Berg and Simms, 1960; Yu et al., 1982). The ability of DR to modulate reproductive aging and slow or prevent the onset of a number of major age-associated diseases, makes diet restriction a tremendously powerful and versatile tool (Yu, 1994b). DR also is used successfully in the poultry industry to achieve the desired body weight without significantly affecting reproductive performance (Fontana et al., 1992). However, the mechanism of action of diet restriction in chickens is not understood.

In animal models with hyperglycemia, DR is effective in retarding the accumulation of the fluorescent end-product, pentosidine, a glycoxidation crosslink in the long-lived tissue protein, collagen, that has been used as biomarker for aging studies (Cefalu et al., 1995). In mammals, pentosidine has been found to increase over the lifespan of the animal; the rate of this accumulation of pentosidine, which is formed by the nonenzymatic glycosylation of lysine and arginine residues, is accelerated in animal models with hyperglycemia (Sell and Monnier, 1989; Grandhee and Monnier, 1991; Dyer et al., 1991a,b; Sell et al., 1992; Reiser, 1994; Nagaraj et al., 1996). 
In addition to DR, aminoguanidine hydrochloride (AG), a nucleophilic hydrazine which acts as a crosslinking inhibitor, delays the formation of collagen-associated crosslinks in the tissues (Brownlee et al., 1988; Khatan et al., 1988; Odetti, 1990; Matuieu et al., 1995; Klandorf et al., 1995). Klandorf et al. (1996) reported that the addition of AG to the diet of broiler breeder hens decreased the accumulation of fluorescent end-products of collagen crosslinks in skeletal muscle. Whether this compound, coupled with industry practices such as diet restriction, can effectively delay the decline in production performance of broiler breeder hens was the basis for this study. The specific objectives of this study were to determine if pentosidine is present in tissue of broiler breeder hens, and to determine if its concentrations change over a production cycle. Because of the links between hyperglycemia, collagen crosslinking, and reproductive function an additional objective was to determine if the crosslinking inhibitor, AG, affected the rate of accumulation of pentosidine and could improve production performance.

\section{MATERIALS AND METHODS}

\section{Birds and Management}

Two studies were undertaken in order to investigate the role of AG in the accumulation of pentosidine in the tissues of broiler breeder hens. In the first study, day-old broiler breeder $\left(\mathrm{CobbXCobb}^{16}\right)$ female $(\mathrm{n}=318)$ and male $(\mathrm{n}=50)$ chicks were placed in electrically heated battery brooders and fed ad libitum until 4 wks of age. All birds were then fed daily with a limited allowance diet according to the Cobb Management Guide and chicks were randomly assigned to one of two experimental groups: control (diet restricted control) or supplemented (diet restricted supplemented with $400 \mathrm{ppm} \mathrm{AG}^{17}$ ) and were fed accordingly throughout the study. At this dose, a highly significant reduction in the accumulation of fluorescent end-products in the skeletal muscle of broiler breeder hens was reported by Klandorf et al. (1996). Roosters were

\footnotetext{
${ }^{16}$ Cobb-Vantress, Inc., Siloam Springs, AR 72761.

${ }^{17}$ Aldrich Chemical Co., Inc., Milwaukee, WI 53233.
} 
reared separately on diet restriction according to the Cobb Management Guide. All the birds were fed daily between 0800 and $1000 \mathrm{hrs}$ and body weight was monitored every two wks. The flocks were reared in floor pens until $20 \mathrm{wks}$ of age. At this time, they were caged individually. At 18 wks the photoperiod was gradually increased over four wks time from 8L:16D to 14L:10D. At 34 wks of age the photoperiod was increased to 15L:9D, for the duration of the study. In a second study, day-old broiler breeder chicks $(n=60)$ were reared as previously described. Since the production performance data in the first study were not significant, production data were not collected.

\section{Pentosidine Determination}

Preparation of Collagen Digest. In both studies, hens $(n=5)$ at 20 wks (before the onset of lay) and 67 to 68 wks of age (end of the production cycle) from both the control and supplemented groups were randomly selected and sacrificed by the industry-standard method of electrical stunning. Approximately $1 \mathrm{~g}$ of skin was removed from the abdominal area, washed with normal saline and stored at $-80^{\circ} \mathrm{C}$ until assayed. The collagen digest used for pentosidine determination was prepared according to a modification of the technique described by Monnier et al. (1986) and Sell et al. (1992). Briefly, this involved the removal of epidermal and adipose layers from skin samples, freezing in liquid nitrogen, and mincing. The minced samples were delipidated overnight in a chloroform-methanol (2:1) solution. Samples were rehydrated in a $50 \%$ methanol solution and hydrolyzed in $6 \mathrm{~N} \mathrm{HCl}$ at $110{ }^{\circ} \mathrm{C}$ for $18 \mathrm{~h}$. All tubes were flushed with $\mathrm{N}_{2}$ prior to capping for heating. Subsequent to the hydrolysis, the samples were placed into a SpeedVac ${ }^{18}$ centrifuge-type vacuum drier until the $\mathrm{HCl}$ was evaporated. Samples were reconstituted in $250 \mu \mathrm{L} \mathrm{H}_{2} \mathrm{O}$ and filtered using a Costar ${ }^{\circledR}$ Spin-X® centrifuge tube filter ${ }^{19}$. A modified Stegman and Stadler spectrophotometric method was used for collagen estimation, using a hydroxyproline standard (Maekawa et al., 1970).

\footnotetext{
${ }^{18}$ Savant Instruments, Farmingdale, NY 11735

${ }^{19}$ Corning Costar Corp., Cambridge, MA 02140
} 
Skin Collagen Pentosidine. The estimation of pentosidine was by a modified reverse phase HPLC method described by Sell et al. (1992). One mg of acid-hydrolyzed collagen digest of skin in $100 \mu \mathrm{L}$ of water/0.01M heptafluorobutyric acid (HFBA) was injected into a 0.46 X25 cm Vydac 218TP104 $(10 \mu \mathrm{m}) \mathrm{C}-18$ column $^{20}$ connected to a Shimadzu HPLC ${ }^{21}$. The apparatus consisted of two model LC-600 pumps, a model SIL-6B auto injector, a model RF 551 fluorescence monitor (excitation $325 \mathrm{~nm}$, emission $370 \mathrm{~nm}$ ). Separations were achieved by application of a linear gradient of $12-42 \%$ acetonitrile from 0 to $20 \mathrm{~min}$ in water and $0.01 \mathrm{M}$ HFBA. Quantitation of pentosidine was made by comparison of peak areas with a 58 pmol pentosidine standard ${ }^{22}$ in $50 \mu \mathrm{L}$ injected under identical conditions. A software package (Shimadzu CLASS-VP 4.2) was used to analyze the data.

\section{Production Trial}

Production Data. Individual egg production and total egg defects (extra large, small, soft, membranous, cracked, thin-shelled) were recorded daily, beginning with the first egg until 70 wks of age. Every 5 wks, beginning with the 25th wk and continuing to the 70th wk, 50 eggs were randomly selected from both groups for determination of eggshell thickness, egg weight, and specific gravity. Eggshell thickness was measured by Digimatic Indicator $543^{23}$ at two sites. Specific gravity was calculated by the computer-assisted displaced-water method (CADW) (Hempe et al., 1988).

Semen Collection, Artificial Insemination, and Fertilization Data. Semen was collected by a modified abdominal massage method (Burrows and Quinn, 1937) from CobbXCobb roosters. Beginning at 30 wks of age, 40 randomly selected hens from each group were artificially inseminated every $10 \mathrm{wks}$ (at 30,40,50,60, and $70 \mathrm{wks}$ ) with $50 \mu \mathrm{L}$ of fresh, undiluted semen. For each time point, artificial insemination was performed weekly beginning

\footnotetext{
${ }^{20}$ Vydac, Hesperia, CA 92345

${ }^{21}$ Shimadzu, Inc., Columbia, MD 21040

${ }^{22}$ Vincent Monnier, Cleveland, OH 44120

${ }^{23}$ Mitutoyo Corp., Japan; Charles Seifert Assoc., Inc., Carnegie, PA 15106 (U.S. reps).
} 
two wks prior to the period in which the fertilization data were to be collected (i.e. beginning insemination at $28 \mathrm{wks}$ for the $30 \mathrm{wk}$ time point). Inseminations were accomplished by the incage technique described by Moultrie (1956).

From each treatment group, fifty eggs were randomly selected from the pool of eggs collected the 3rd and 4th days following each insemination and incubated in a forced-air incubator $^{24}$ at $37.5{ }^{\circ} \mathrm{C}$ and $60 \%$ relative humidity. The eggs were removed from the incubator after seven days for testing of fertilization and viability. Fertilization was calculated as the number of fertilized eggs per 50 eggs set. Embryo viability was calculated as the number of viable embryos on day 7 of incubation per 50 fertilized eggs. Embryo production was measured as the number of viable embryos on day 7 of incubation per 50 eggs set (fertilization x embryo viability) (Robinson et al., 1991).

\section{Statistical Analyses}

One way ANOVA and Fisher's LSD test were done to compare the control and supplemented groups for each production parameter at each time point. The interaction between age and dietary groups for pentosidine was analyzed by a two-way ANOVA (SAS Institute, 1990).

\section{RESULTS AND DISCUSSION}

The Maillard hypothesis of aging proposes that aging results, in part, from chronic chemical modifications of proteins and other biomolecules by reducing sugars and by the accumulation of glycoxidation products and other advanced glycosylation end-products (AGEs) in tissue protein (Cerami, 1985; Monnier, 1990; Reiser, 1991). Because lifespan has been extended by DR in lab animals, we investigated the effect of DR, with supplementation of AG, on the formation of AGEs in the collagen of aging broiler breeder hens. The extent of crosslinking was quantified by measurement of the fluorescent end-product, pentosidine. While

\footnotetext{
${ }^{24}$ Jamesway Incubatory Co. Monroe, NC 28110
} 
this compound has been characterized in mammals, no investigations involving pentosidine have been reported in birds prior to this study. In agreement with results from mammalian studies (Dunn et al., 1989, 1991; Koizumi et al., 1989; Sell and Monnier, 1989, 1990; Dyer et al, 1991a,b; Sell et al., 1992; Reiser, 1994; Cefalu et al., 1995; Nagaraj et al., 1996), the glycoxidation product, pentosidine was detected in the tissues of birds. We also found that the accumulation of pentosidine in skin collagen of control and supplemented broiler breeder hens increased with age $(\mathrm{P}<0.001)$ (Fig. 1). Skin pentosidine increased $75 \%$ and $78 \%$ in the control and supplemented groups, respectively. Aminoguanidine $(\mathrm{P}<0.001)$ retarded the accumulation of skin pentosidine (Fig. 1). Comparable results have been reported in mammals (Brownlee et al., 1986; Hayase et al., 1991; Odetti, 1990; Edelstein and Brownlee, 1992 a,b; Oxuland and Andreassen, 1992; Yagihashi et al., 1992; Wu, 1995).

In spite of the elevated metabolism and plasma concentrations of glucose in birds (Holmes and Austad, 1995), the measured concentrations of pentosidine were markedly lower in diet-restricted broiler breeder hens as compared to age-corrected diet-restricted mammals (Sell and Monnier, 1989; Cefalu et al., 1995). In a comparable study, the concentration of glycosylated hemoglobin $(\mathrm{GHb})$ in hummingbirds is lower as compared to mammals in spite of circulating concentrations of plasma glucose in excess of $650 \mathrm{mg} / \mathrm{dL}$ (Beuchat and Chong, 1997). It appears that the bird has evolved mechanisms to limit dramatically the rate of accumulation of these crosslinks. It has been hypothesized that the mechanisms of free radical production and antioxidant defenses differ between birds and mammals (Holmes and Austad, 1995). Conlon et al., (1991) reported that oxygen free radical production in avian (Red-tailed hawk and chicken) heterophils was significantly lower than in analogous bovine neutrophils. In a recent study using pigeons and rats, $\mathrm{Ku}$ and Sohal (1993) found that mitochondrial generation of both oxygen free radicals and peroxide was lower in pigeons, and that pigeons had higher antioxidant activities. Antioxidants such as vitamin A (retinol and retinyl esters) and $\alpha$-tocopherol (vitamin E), have been found at higher concentrations in a variety of birds than in mammals (Schweigert et al., 1991). In addition, uric acid and its' anion form, urate, are recognized as ubiquitous antioxidants in humans, monkeys, and insects (Souza et al., 1996; Hellsten et al., 1996). Because the 
concentration of plasma uric acid in birds ( 8 to $12 \mathrm{mg} / \mathrm{dL}$; Shapiro et al., 1997) is approximately two-fold greater than the urate concentrations measured in humans (2.6 to $7.2 \mathrm{mg} / \mathrm{dL}$; Bishop et al., 1992), it may be speculated that the lower tissue concentrations of the glycoxidation product, pentosidine, in hens as compared to mammals is due to a more efficacious avian antioxidant system. Further exploration into this area may contribute to the understanding of the aging process.

Although AG effectively reduced skin pentosidine there were no consistent effects of AG treatment on the reproductive cycle. Production data (egg production, egg weight, eggshell thickness, egg defects, and specific gravity) in birds fed AG did not differ consistently from that of the control animals (Fig. 2, Tables 1 and 2). Fertility, viability and embryo production also were not significantly different between the two treatments (Table 3). It appears that the dose of AG (400 ppm) is effective in lowering the pentosidine from the skin collagen but not in ovarian collagen which suggests the use of higher AG dosage. Alternatively, there could be a negative correlation between increase of skin pentosidine concentrations and decrease in egg production over the period of time.

In conclusion, although supplementation of diet restricted broiler breeder hens with AG retarded the rate of accumulation of pentosidine over a laying cycle, the reduction in pentosidine did not significantly affect mean production performance.

\section{Acknowledgments}

The authors wish to thank Vincent M. Monnier, Institute of Pathology, Case Western Reserve University for supplying the pentosidine standard and other technical guidance. We are also grateful to Nabil Al-Humadi, NIOSH for his valuable assistance in HPLC procedure, Edwin C. Townsend for his assistance in statistical analysis and Christine Dezerte, DESS Inovation Tecnologique et union Europeenne, France for her assistance in the analyses of production parameters. 


\section{REFERENCES}

Beuchat, C. A., and C. R. Chong, 1997. Hyperglycemia in hummingbirds: Implications for hummingbird ecology and human health. FASEB J. 11:3:A91.

Berg, B. N., and H. S. Simms, 1960. Nutrition and longevity in the rat. II. Longevity and onset of disease with different levels of food intake. J. Nutr. 71:255-259.

Bishop, M. L., J. L. Duben-Engelkink, E. P. Fody, 1992. Clinical chemistry, principles, procedures, correlations. 2nd. Ed. Philadelphia, PA: Lippincott.

Brownlee, M., H. Vlassara, A. Kooney, P. Ulrich, A. Cerami, 1986. Aminoguanidine prevents diabetes-induced arterial wall protein cross-linking. Sci. 232: 1629-1632.

Brownlee, M., A. Cerami, and H. Vlassara, 1988. Advanced products of nonenzymatic glycosylation and the pathogenesis of diabetic vascular disease. Diabetes Metabolism Reviews. $4: 437-451$

Burrows, W. H., and J. P. Quinn, 1937. The collection of spermatozoa from the domestic fowl and turkey. Poultry Sci. XVI:19-24.

Cefalu, W. T., A. D. Bell-Farrow, Z. Q. Wang, W. E. Sonntag, M. Fu., J. W. Baynes and S. R. Thorpe, 1995. Caloric restriction decreases age-dependent accumulation of the glycoxidation products, N-(Carboxymethyl)lysine and pentosidine, in rat skin collagen. J. Geront: Biol. Sci. 50A:B337-B341.

Cerami, A., 1985. Hypothesis: glucose as a mediator of aging. J. Am. Geriatr. Soc. 33: 626-634.

Conlon, P., D. Smith, T. Gowlett, 1991. Oxygen radical production by avian leukocytes. Can. J. Vet. Res. 55:193-195. 
Dunn, J. S., J. S. Patrick, S. R. Thrope, J. W. Bayens, 1989. Oxidation of glycated protein: age dependent accumulation of $\mathrm{N}$-(Carboxymethyl)lysine in lens proteins. Biochemistry 28: 94649468.

Dunn, F.A., D. R. McCance, D. R. Thorpe, T. J. Lyons, J. W. Baynes, 1991. Age-dependent accumulation of $\mathrm{N}$-(carboxymethyl)lysine and $\mathrm{N}$-(Carboxymethyl)hydroxylysine in human skin collagen. Biochemistry 30:1205-1210.

Dyer, D. G., J. A. Blackledge, S. R. Thorpe, J. W. Baynes, 1991a. Formation of pentosidine during nonenzymatic browning of proteins by glucose. J. Biol. Chem. 266:11554-1660.

Dyer, D. G., J. A. Blackledge, J. R. Thorpe, and F. W. Baynes, 1991b. Formation of pentosidine during nonenzymatic browning of proteins by glucose: identification of glucose and other carbohydrates as possible precursors of pentosidine in vivo. J. Biol. Chem. 266:11654-60.

Edelstein, D., and M. Brownlee, 1992a. Mechanistic studies of advanced glycosylation end product inhibition by aminoguanidine. Diabetes 41:26-29.

Edelstein, D., and M. Brownlee, 1992b. Aminoguanidine ameliorates albuminuria in diabetic hypertension rats. Diabetologia, 35:96-97.

Fontana, E. A., W. D. Weaver, Jr., B. A. Watkins, and D. M. Denbow, 1992. Effect of early feed restriction on growth, feed conversion and mortality in broiler chickens. Poultry Sci. 72: 243250.

Grandhee, S. K., and V. M. Monnier, 1991. Mechanisms of formation of the Maillard protein cross-link pentosidine: ribose, glucose, fructose and ascorbate as pentosidine precursors. J. Biol. Chem. 266: 11649-53. 
Hayase, F., Y. H. Kim, and H. Kato, 1991. Inhibition of the protein crosslinking in a Maillard reaction by aminoguanidine and Semicarbazide. Agric. Biol. Chem. 55:1435-1436.

Hellsten, Y., P. C. Tullson, E. A. Richter, J. Bangsbo, 1997. Oxidation of urate in human skeletal muscle during exercise. Free Radical Biol. and Med. 22:169-174.

Hempe, J. M., R. C. Lauxen, and J. E. Savage, 1988. Rapid determination of egg weight and specific gravity using a computerized data collection system. Poultry Sci. 67:902-907.

Holmes, J. D., and S. N. Austad, 1995. Birds as animal models for the comparative Biology: A prospectus. J. Geront: Biol. Sci. 50A:B59-B66.

Kaiser, F. E., and S. G. Korenman, 1988. Impotence in diabetic men. Amer. J. Med. 85:147152.

Khatan, M., Z. Soldan, I. David, W. Li, and J. H. Rockey, 1988. Inhibitory effects of pyridoxal phosphate, ascorbate and aminoguanidine on nonenzymatic glycosylation. Life Sci. 43:17251731.

Klandorf, H., S. B. Holt, J. A. McGowan, Y. Pinchasov, D. Deyette, and R. A. Peterson, 1995. Hyperglycemia and non-enzymatic glycation of serum and tissue proteins in chickens. Comp. Biochem. Physiol. 110C:215-220.

Klandorf, H., Q. Zhou, and A. R. Sams, 1996. Inhibition by aminoguanidine of glucose-derived collagen cross-linking in skeletal muscle of broiler breeder hens. Poultry Sci. 75:432-437.

Koizumi, A., Y. Wada, M. Tsukada, J. Hasegawa, 1989. Low blood glucose levels and small Islets of Langerhans in the pancreas of caloric-restricted mice. Age 12:93-96.. 
Ku, H., and R. S. Sohal, 1993. Comparison of mitochondrial prooxidant generation and antioxidant defenses between rat and pigeon: possible basis of variation in longevity and metabolic potential. Mech. Aging Dev. 72:67-76.

Lerner, L. P., N. French, D. McIntyre, and C. Baxter-Jones, 1993. Age-related changes in egg production, fertility, embryonic mortality, and hatchability in commercial turkey flocks. Poultry Sci. 72:1025-1039.

Maekawa, T., Y. K. Ratinasamy, K. I. Altman and W. F. Forbes. 1970. Changes in collagen with age-I. The extraction of acid soluble collagens from skin of mice. Exp. Geront. 5:177-186.

Mathieu, C., M. Waer, K. Casteels, J. Laureys, and R. Bouillon, 1995. Prevention of Type I diabetes in NOD mice by non-hypercalcemic doses of a new structural analog of 1, 25dihydroxyvitamin D3, DH 1060. Endocrinology 136:866-872.

Monnier, V. M., V. Vishwanath, K. E. Frank, C. A. Elmets, P. Dauchot and P. R. Kohn, 1986. Relationship between complications of type I diabetes mellitus and collagen-linked fluorescence. N. Engl. J. Med. 314:403-408.

Monnier, V. M.1990. Nonenzymatic glycosylation, the Maillard reaction and the aging process. J. Gerontol: Biol. Sci. 45: B105-B111.

Moultrie, F., 1956. A new technique for the artificial insemination of caged hens. Poultry Sci. 35:1230-1234.

Nagaraj, R. H., T. S. Timothy, D. R. Sell, J. Fogarty, R. L. Engerman, and V. M. Monnier, 1996. Evidence of a glycemic threshold for the formation of pentosidine in diabetic dog lens but not in collagen. Diabetes 45:567-594. 
Odetti, P. R., A. Borgoglio, A. D. Pascale, R. Polandi and L. Adezati, 1990. Prevention of diabetes-increased aging effect on rat collagen-linked fluorescence by aminoguanidine and rutin. Diabetes. 39:796-801.

Oxuland, H. and T. T. Andreassen, 1992. Aminoguanidine treatment reduces increase in collagen stability of rats with experimental diabetes mellitus. Diabetologia 35: 19-25.

Reiser, K. M., 1991. Nonenzymatic glycation of collagen in aging and diabetes. Prec. Soc. Exp. Biol. Med. 196:17-29.

Reiser, K. M., 1994. Influence of age and long-term dietary restriction on enzymatically mediated crosslinks and nonenzymatic glycation of collagen in mice. J. Gerontol: Biol.Sci. 49:B71-B79.

Robinson, F. E., R. T. Hardin, and B. J . Williams, 1991. The influence of egg sequence position on fertility, embryo viability, and embryo weight in broiler breeders. Poultry Sci. $70: 760-765$.

SAS Institute, 1990. SAT/STAT ${ }^{\circledR}$ User's Guide: Statistics. Release 6.04 SAS Institute Inc., Cary, NC.

Schweigert, F.F., S. Uehlein-Harrell, G. Von Hegel, H. Wiesner, 1991. Vitamin A (retinol and retinyl esters), $\alpha$-tocopherol and lipid levels in plasma of captive wild mammals and birds. Xentralblatt fur Veterinarmedizin-Reihe A 38:35-42.

Sell, D. R., V. M. Monnier, 1989. Structural elucidation of senescence cross-link from human cross-link from human extra-cellular matrix: Implication of pentoses in the aging process. J. Biol. Chem. 264:21597-21602. 
Sell, D. R., and V. M. Monnier, 1990. End-state renal disease and diabetes catalyze the formation of a pentose-derived cross-link from aging human collagen. J. Clin. Invest. 85:380-84.

Sell, D. R., A. Lapolla, P. Odetti, J. Fogarty, and V. M. Monnier, 1992. Pentosidine formation in skin correlates with severity of complications in individuals with long-standing IDDM. Diabetes, 41:1286-1291.

Shapiro, F., M. Mahagna, I. Nir, 1997. Stunting syndrome in broilers: effect of glucose or maltose supplementation on digestive organs, intestinal disaccharidase, and some blood metabolites. Poultry Sci. 76:369-380.

Souza, A. V. G., J. H. Petretski, M. Demasi, E. J. H. Bechara, P. L. Oliveira, 1997. Urate protects a blood-sucking insect against hemin-induced oxidative stress. Free Radical Biol. and Med, 22:209-214.

Wu, G. 1995. Nitric oxide synthesis and effect of aminonguanidine and N-monomethyl-Larginine on the onset of diabetes in spontaneously diabetic BB rats. Diabetes, 44:36-365.

Yagihashi, S., M. Kamigo, M. Baba, N. Yahishi, and N. Nagai, 1992. Effect of aminoguanidine on functional and structural abnormalities in peripheral nerve of STZ-induced diabetic rats. Diabetes, 41:47-52.

Yu, B. P., E. J. Masoro, I. Murafa, H.Q. Berfrand and F.T. Lynd, 1982. Life-span study of SPF Fischer 344 male rats fed ad libitum or restricted diets: longevity, growth, lean body mass and disease. J. Gerontol. 37:130.

Yu, B. P. 1994b. How diet influences the aging process of the rat. Proc. Soc. Exp. Biol. Med. 205:97-107. 
Table 1: Egg production and egg defects (Mean \pm SEM)

\begin{tabular}{ccccccc}
\hline \hline \multirow{2}{*}{$\begin{array}{c}\text { Age } \\
(\text { wks })\end{array}$} & \multicolumn{2}{c}{ H } & \multicolumn{2}{c}{ Hen Day Egg Production (\%) } & \multicolumn{2}{c}{ Egg Defects (\%) $\dagger$} \\
\hline 25 & 106 & $34 \pm 2$ & 112 & $33 \pm 2$ & $46 \pm .3 *$ & $51 \pm .3$ \\
30 & 105 & $80 \pm 3$ & 111 & $79 \pm 3$ & $11 \pm .3$ & $6 \pm .2 *$ \\
35 & 105 & $75 \pm 3$ & 110 & $73 \pm 3$ & $10 \pm .3$ & $9 \pm .2$ \\
40 & 104 & $75 \pm 3 *$ & 110 & $70 \pm 3$ & $2 \pm .2$ & $2 \pm .2$ \\
45 & 103 & $71 \pm 3$ & 109 & $71 \pm 3$ & $1 \pm .1$ & $.49 \pm 0$ \\
50 & 102 & $66 \pm 2^{*}$ & 107 & $60 \pm 3$ & $5 \pm .2$ & $4 \pm .1$ \\
55 & 101 & $58 \pm 2$ & 106 & $63 \pm 2 *$ & $3 \pm .1$ & $2 \pm .1$ \\
60 & 101 & $51 \pm 2$ & 106 & $48 \pm 2$ & $4 \pm .1$ & $5 \pm .1$ \\
65 & 100 & $45 \pm 2$ & 106 & $44 \pm 2$ & $3 \pm .1 *$ & $6 \pm .2$ \\
70 & 99 & $41 \pm 3$ & 105 & $39 \pm 3$ & $3 \pm .1 *$ & $7 \pm .2$ \\
\hline
\end{tabular}

$* \mathrm{P}<0.05$ between dietary groups at those time points only

Control $=$ diet restricted control

Suppl. $=$ diet restricted supplemented with $400 \mathrm{ppm}$ aminoguanidine

$\dagger \%$ of extra large, small, soft, thin-shelled, membranous, deformed, cracked eggs. 
Table 2: Eggshell thickness, egg weight, and specific gravity (Mean \pm SEM)

\begin{tabular}{cccccccc}
\hline \hline \multirow{2}{*}{$\begin{array}{c}\text { Age } \\
\text { (wks) }\end{array}$} & $\mathrm{n}$ & \multicolumn{2}{c}{ Shell thickness (mm) } & \multicolumn{2}{c}{ Egg weight (g) } & \multicolumn{2}{c}{ Specific gravity } \\
\cline { 2 - 7 } & Control & Suppl. & Control & Suppl. & Control & Suppl. \\
\hline 25 & 50 & $.36 \pm 0.003$ & $.36 \pm .0003$ & $52.9 \pm 0.94$ & $49.1 \pm 0.94$ & $1.083 \pm 0.001$ & $1.081 \pm 0.001$ \\
30 & 50 & $.35 \pm 0.004$ & $.34 \pm 0.004$ & $58.7 \pm 0.51$ & $57.9 \pm 0.51$ & $1.080 \pm 0.001$ & $1.083 \pm 0.001$ \\
35 & 50 & $.38 \pm 0.004$ & $.37 \pm 0.004$ & $62.1 \pm 0.60$ & $61.0 \pm 0.60$ & $1.080 \pm 0.001$ & $1.079 \pm 0.001$ \\
40 & 50 & $.38 \pm 0.003$ & $.37 \pm 0.003$ & $62.6 \pm 0.85$ & $64.8 \pm 0.85$ & $1.079 \pm 0.002$ & $1.082 \pm 0.002$ \\
45 & 50 & $.35 \pm 0.003$ & $.36 \pm 0.003$ & $63.8 \pm 0.60$ & $63.8 \pm 0.60$ & $1.078 \pm 0.006$ & $1.078 \pm 0.006$ \\
50 & 50 & $.37 \pm 0.004$ & $.39 \pm 0.004$ & $68.1 \pm 0.56$ & $67.0 \pm 0.56$ & $1.076 \pm .0002^{*}$ & $1.062 \pm 0.002$ \\
55 & 50 & $.36 \pm 0.004$ & $.37 \pm 0.004$ & $68.0 \pm 0.61$ & $67.6 \pm 0.61$ & $1.061 \pm 0.001$ & $1.062 \pm 0.001$ \\
60 & 50 & $.32 \pm 0.003 *$ & $.29 \pm 0.003$ & $69.6 \pm 0.55 *$ & $66.5 \pm 0.55$ & $1.065 \pm 0.001$ & $1.071 \pm 0.001 *$ \\
65 & 50 & $.37 \pm 0.003$ & $.38 \pm 0.004$ & $72.4 \pm 0.47 *$ & $70.0 \pm 0.47$ & $1.049 \pm 0.001$ & $1.048 \pm 0.001$ \\
70 & 50 & $.34 \pm 0.003$ & $.34 \pm 0.003$ & $72.4 \pm 0.47 *$ & $70.0 \pm 0.47$ & $1.048 \pm 0.00$ & $1.047 \pm 0.003$ \\
\hline
\end{tabular}

$* \mathrm{P}<0.05$ between dietary groups at those time points only

Control $=$ diet restricted control

Suppl. $=$ diet restricted supplemented with 400 ppm aminoguanidine

Table 3: Fertility, viability and embryo production (Mean \pm SEM)*

\begin{tabular}{llcccccc}
\hline \hline \multirow{2}{*}{$\begin{array}{c}\text { Age } \\
\text { (wks) }\end{array}$} & $\mathrm{n}$ & \multicolumn{2}{c}{ Fertility (\%) } & \multicolumn{2}{c}{ Viability (\%) } & \multicolumn{2}{c}{ Embryo production (\%) } \\
\cline { 2 - 7 } & Control & Suppl. & Control & Suppl. & Control & Suppl. \\
\hline 30 & 50 & $94 \pm 2$ & $88 \pm 2$ & $94 \pm 1$ & $84 \pm 1$ & $94 \pm 1$ & $84 \pm 2$ \\
40 & 50 & $100 \pm 2$ & $96 \pm 2$ & $100 \pm 1$ & $94 \pm 1$ & $100 \pm 2$ & $94 \pm 2$ \\
50 & 50 & $98 \pm 2$ & $96 \pm 2$ & $98 \pm 1$ & $90 \pm 1$ & $96 \pm 2$ & $90 \pm 2$ \\
60 & 50 & $76 \pm 2$ & $74 \pm 2$ & $68 \pm 1$ & $74 \pm 1$ & $68 \pm 1$ & $74 \pm 1$ \\
70 & 50 & $72 \pm 2$ & $70 \pm 2$ & $66 \pm 1$ & $64 \pm 1$ & $66 \pm 1$ & $66 \pm 1$ \\
\hline
\end{tabular}

* Non significant difference between dietary groups $(\mathrm{P}>0.05)$

Control $=$ diet restricted control

Suppl. $=$ diet restricted supplemented with 400 ppm aminoguanidine 
Figure 1. Skin pentosidine in Control and AG-supplemented groups. Data is the mean of five birds. $* * * \mathrm{P}<0.001$.

Figure 2. Hen day egg production in Control and AG-supplemented groups. 
Figure 1

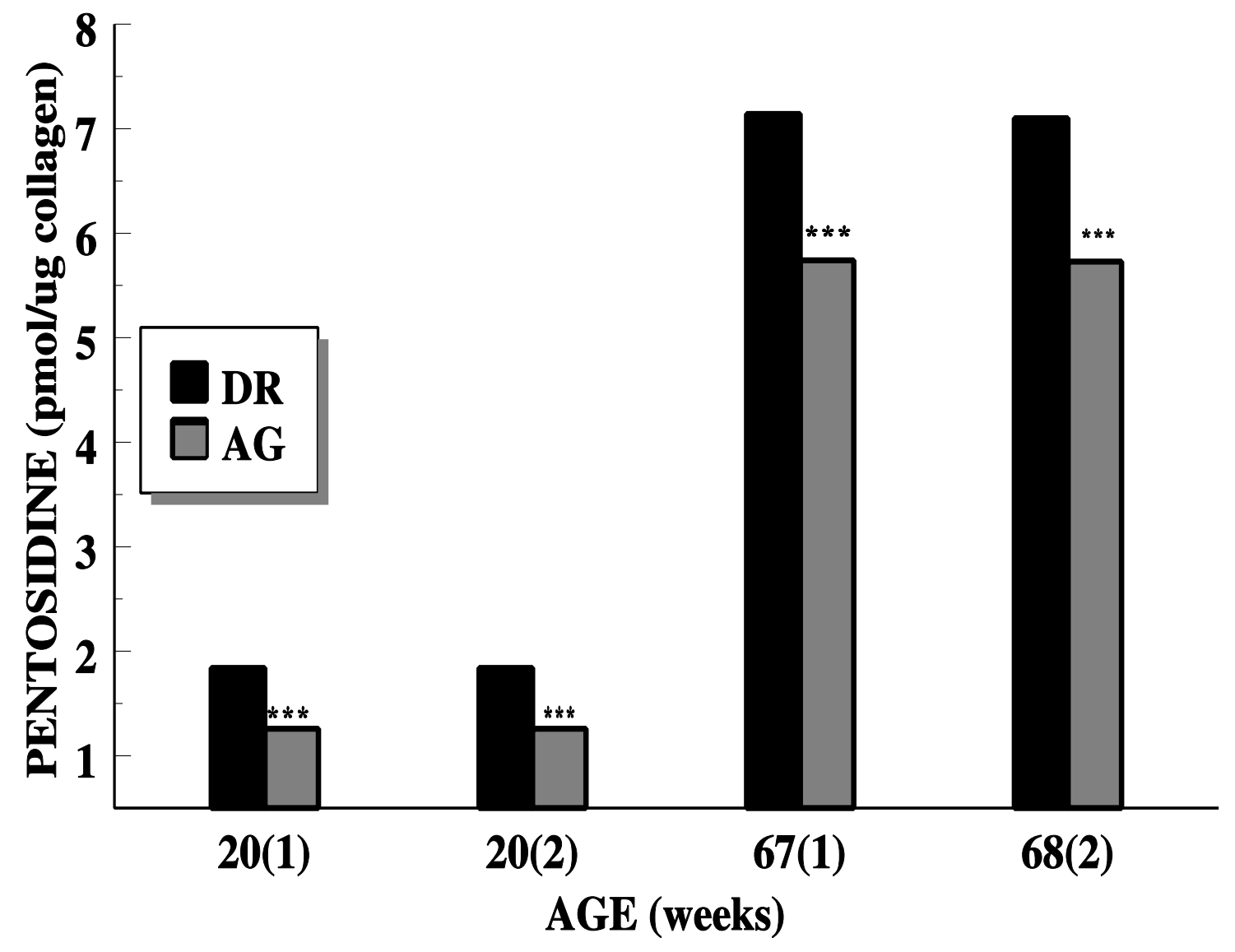


Figure 2

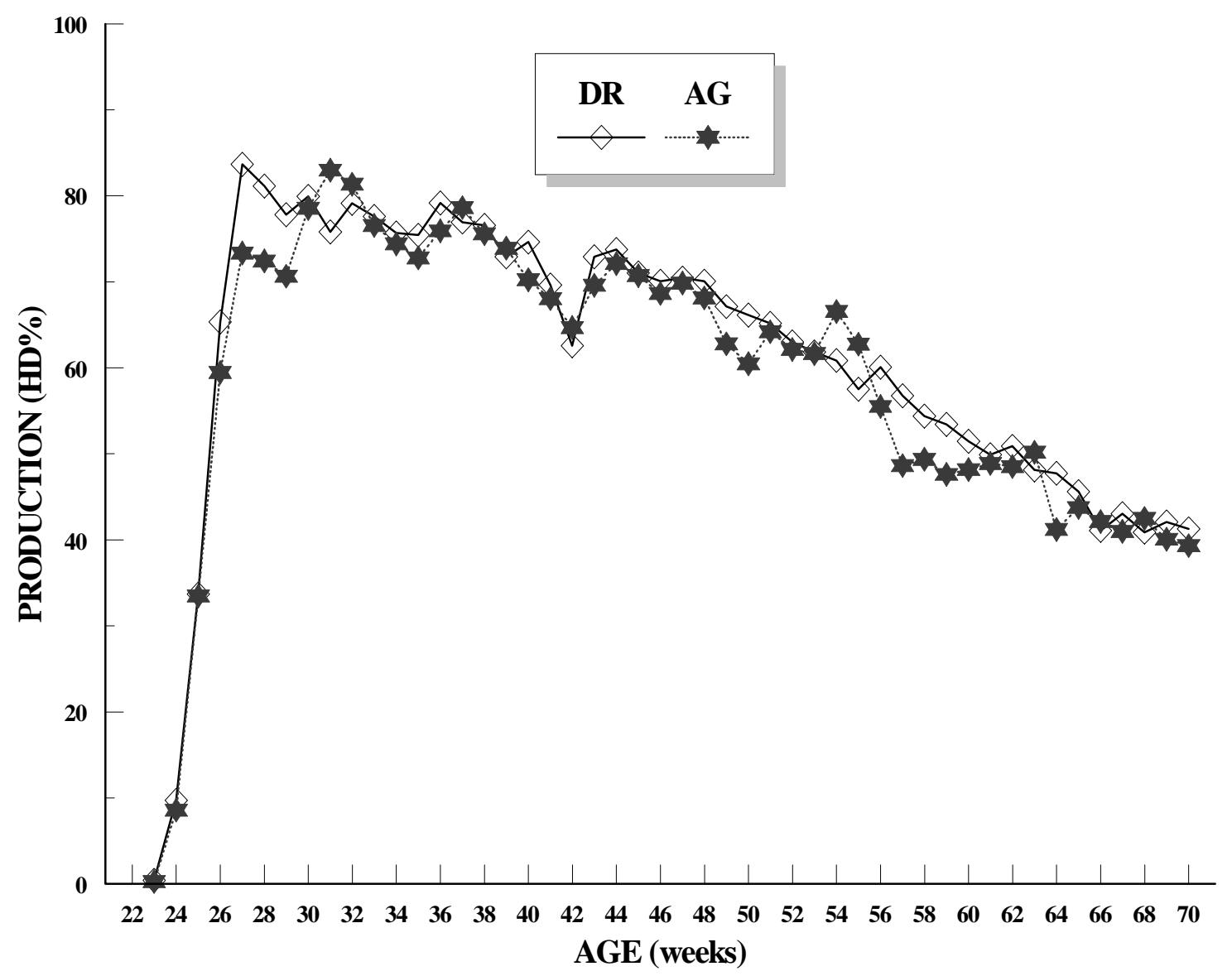




\section{Chapter 4}

\section{SUMMARY}

The factors which influence the age-related decline in production performance and meat toughness in broiler breeder hens are poorly understood. A characteristic of broiler breeder hens is the rapid, age-related decline in production performance once the genetic potential for maximum production of eggs has been reached at 32 weeks. In addition, the meat of aged hens becomes objectionably tough, limiting its use in whole muscle food. Broiler breeder hens are characterized by both obesity and hyperglycemia (plasma glucose concentrations 2-4 fold higher than humans). We have hypothesized that the elevated plasma glucose concentrations contribute to the nonenzymatic attachment of glucose to proteins and generate glycoxidation crosslinks in tissues which can ultimately impair their function. One such crosslink, pentosidine, is an established indicator of both glycative and oxidative tissue damage in mammals. Because of its linear, age-related accumulation on the structural protein, collagen pentosidine can also be used as a biomarker for the study of aging. Diet restriction (DR) is the only known reliable measure for slowing the glycosylation process and, subsequently, the formation of advanced glycosylation endproducts (AGEs) in tissues. Specifically, we examined the role of DR and a nucleophilic hydrazine compound, aminoguanidine (AG), a crosslinking inhibitor, on tissue crosslinking with the view towards both extending the production period and improving carcass quality of broiler breeder hens. The objectives of these studies were to determine if: 1) pentosidine is present in the skin $\left(\mathrm{P}_{\mathrm{s}}\right)$ and tendon $\left(\mathrm{P}_{\mathrm{t}}\right)$ of broiler breeder hens (isolation and quantification) ; 2) pentosidine accumulates over the the life time of the animal; 3) dietary modulation and supplementation with the crosslinking inhibitor, aminoguanidine (AG), could retard the accumulation of pentosidine, improve production performance, and limit the agerelated increase in toughness of meat and tendon. Chicks were randomly divided into four dietary groups from 8 to 125 wks after hatch: ad libitum (AL); diet restricted (DR, fed 60\% of ad libitum); AL and DR groups supplemented with 400 ppm AG (1.35 mg/kg BW/day) each 
(AL+ AG and DR+AG respectively) for studies I, II and III. Two experiments (study IV) were carried ( $\mathrm{n}=318 ; 60$ respectively) were carried out for reproductive performance (\%HD egg production, egg defects, egg shell thickness, egg weight, specific gravity, fertility, viability, embryo production) until $70 \mathrm{wk}$ of age. Collagen estimation was done by a hydroxyproline spectrophotometric method. Pentosidine was isolated and quantified by reversed phase HPLC. Plasma glucose and uric acid concentrations were estimated by standardized procedures. Oxidative stress was determined by a modified luminometric method. Tenderness of pectoralis major muscle was measured in terms of shear force (SF) using an Instron Universal Mechanical Machine. Tendon (flexor perforatus et perforans digiti iii) breaking time (TBT) and strength (TBS) were also determined by developing a new technique using an Instron Universal Mechanical Testing Machine. Egg production and egg defects were recorded daily. Egg shell thickness, egg weight, specific gravity $(n=50)$ were measured every 5 wks. Fertility, viability and embryo production trials were carried out on fertile eggs obtained from artificially inseminated hens at an interval of $10 \mathrm{wk}$ starting from 30th wk until 70th wk.

The results showed that pentosidine was present in the skin and tendon of the broiler breeder hens, and that there was an age-related, linear increase in its concentration $(p<0.001$, $\left.r^{2}=0.94\right)$ for skin and $\left(p<0.0001 ; r^{2}=0.79\right)$ for tendon in AL-fed hens. DR and supplementation of AG with AL-fed hens (AL+AG) retarded $\mathrm{P}_{\mathrm{s}}(p<0.001 ; p<0.001)$ and $\mathrm{P}_{\mathrm{t}}(p<0.001 ; p<0.01)$ respectively as compared to AL-fed hens. Concurrently, there was also an age-related parallel increase in TBS $\left(p<0.0001 ; r^{2}=0.56\right)$, and in TBT $(p<0.0001 ; r=0.67)$ in the same hens. Similarly, DR significantly retarded the overall TBS $(p<0.01)$ and TBT $(p<0.02)$ values as compared to AL-fed hens. Oxidative stress was lower $(p<0.0001)$ and plasma uric acid higher ( $p<0.0003)$ in both the $\mathrm{DR}$ and $\mathrm{DR}+\mathrm{AG}$ groups than in the $\mathrm{AL}$ and $\mathrm{AL}+\mathrm{AG}$ groups. There was a linear age-related increase in SF $\left(p<0.0001 ; r^{2}=0.95\right)$, cook time (CT) $\left(p<0.0001 ; r^{2}=0.95\right)$ and breast weight $(\mathrm{Br} . \mathrm{W})\left(p<0.0001 ; r^{2}=0.64\right)$ in AL-fed hens. DR significantly retarded SF $(p<0.0001)$ throughout the study. Age-related linear increase in SF correlated $\left(r^{2}=0.86\right)$ with age-related increase in $\mathrm{P}_{\mathrm{s}}$ in $\mathrm{AL}-\mathrm{fed}$ hens. In the $\mathrm{AL}+\mathrm{AG}$ group, $\mathrm{SF}$ values were similar to those of the DR group. Although, supplementation of DR with $A G(D R+A G)$ retarded the rate of 
accumulation of $\mathrm{P}_{\mathrm{s}}(p<0.001)$ over the laying cycle, the reduction in $\mathrm{P}_{\mathrm{s}}$ did not significantly affect mean production. It is therefore concluded that $\mathrm{P}_{\mathrm{s}}, \mathrm{P}_{\mathrm{t}}, \mathrm{TBS}, \mathrm{TBT}$ and the age-related increase in meat toughness can be retarded by either DR or AG. $\mathrm{P}_{\mathrm{s}}$ can also be used as a predicator of age-related changes in meat. The same approach has the potential to be initiated in other animal production systems. Because of the linear, age-related increase in the concentrations of tissue pentosidine, it can be used as biomarker for the study of aging. 


\section{Bibliography}

Ames, B.N., Cathcart, R., Schwiers, E., Hochstein, P. 1981. Uric acid provides an antioxidant defense in humans against oxidant- and radical-caused aging and cancer: A hypothesis. Proc. Natl. Acad. Sci. U.S.A. 78:6858-6862.

Ames, B.N., Shigenaga, M.K., and Hagen, T.M. 1993. Oxidants, antioxidants, and the degenerative diseases of the aging. Proc. Natl. Acad. Sci. USA 90:7915.

Amudeswari, S., and Liang, J.N., Chakrabarti, B. 1987. Polar-apolar characteristics and fibrillogenesis of glycoslylatd collagen. Collagen Relat. Res. 7:215-223.

Andreassen, T.T., Seyer-Hansen, K., and Oxlund, H. 1981. Biomechanical changes in connective tissues induced by experimental diabetes. Acta Endocrinol. 98:432-436.

Andreassen T.T., and Oxlund, J. 1985 Thermal stability of collagen in relation to non-enzymatic glycosylation and browning in vitro. Dabetologia 28:687-69.

Andreassen, T.T., and Oxlund, H. 1987. Changes in collagen and elastin of rat aorta induced by experimental diabetes and food restriction . Acta Endocrionl. 98: 432-436.

Andreassen, T.T., Oxlund, J., and Danielsen, C.C. 1988. The influence of nonenzymatic glycosylation and formation of fluorescent reaction products on the mechanical properties of rat tail tendons. Conn Tissue Res. 17:1-9.

Aoki, Y., Yanagisawa, Y., Yazaki, K., Oguchi, H., Kiyosawa, K., and Furuta, S. 1992. Protective effect of vitamin E supplementation on increased thermal stability of collagen in diabetic rats. Diabetologia 35:913-916.

Arganosa, G.C., and Marriott, N.G. 1989. Organic acids as tenderizers of collagen in restructured beef. J. Food Sci. 54:1173-1175.

Aust, S.D., Morehouse, L.A.L, and Thomas, C.E. 1985. Role of metals in oxygen radical reactions. J. Free Radic. Biol. Med. 1:3.

Austad, S., and Fischer, K. 1991. Mammalian aging, metabolism, and ecology: evidence from bats and marsupials. J. Gerontol: Biol. Sci. 46:B47-B53.

Awonorin, S.O., and Ayoade, J. A., 1992. Texture and eating quality of raw- and thawed- roasted turkey and chicken breasts as influenced by age of birds and period of frozen storage. J. Foodserv. Syst. 6: 241-255. 
Bailey, A. J., and Light, N.D. 1989. The structure and growth of muscle. Pages 27-47 In: Meat Science. R. A. Lawrie. ed. 5th, New York.

Bailey, A.J., Sims, T.J., Avery, N.C., and Halligan, E.P. 1995. Nonenzymatic glycation of fibrous collagen: reaction products of glucose and risbose. Biochem. J. 305:385-390.

Bandy, B. and Davison, A. 1990. Mitochondrial mutations may increase oxidative stress: implications for carcinogenesis and aging? Free Radic. Biol. Med. 8:523.

Barja, G., Lopez-Torres, M., and Perez-Campo, R. 1992. Relationship between antioxidants, lipid peroxidation and aging. I. Emerit and B. Chance, eds. In: Free Radicals and Aging. Basel, Switzerland, Birkhauser Verlag. pp. 109-123.

Barja, G., Cadenas, S., Rojas, C., Perez-Compo, R., and Lopez-Torrez, M. 1994. Low mitochondrial free radical production per unit of consumptions can explain the simultaneous

presence of high longevity and high aerobic metabolic rate in birds. Free Radic. Res. 21:37-328.

Batcher, O.M., Dawson, E.H., Pointer, M.T., and., Gilpin, G.L. 1962. Quality of raw and cooked lamb meat as related to fatness and age of animal. Food Technol. 16:102-110.

Baynes, J.W., Monnier, V.M. 1989. eds. The Maillard reaction in aging, diabetes and nutrition. In: Progress in clinical biology research. New York Alan R. Liss.

Baynes, J.W. 1991. Role of oxidative stress on development to complications in diabetes. Diabetes 40: 405-412.

Becker, F.B. 1993. Towards the physiological function of uric acid. Free Radic. Biol. Med. 14:615-631.

Bensusan, H.B. 1965. A novel hypothesis for the mechanism of crossliniking in collagen: Structure and chemistry of collagen. In: structure and Function of connective and Skeletal Tissue. Harkness N.D., Partridge, S.M., Tristiam, D.R. Eds. Butteworth, London, P. 42-46.

Berg, B.N., and H.S. Simms, 1960. Nutrition and longevity in the rat. II. Longevity and onset of disease with different levels of food intake. J. Nutr. 71:255-259.

Berg, T. 1996. Increased serum levels of advanced glycosylation end products (AGEs) in children and adolescents with IDDM. Diabetes Abstracts.

Beuchat, C.A., and Chong, C.R. 1997. Hyperglycemia in hummingbirds: Implications for hummingbird ecology and human health. FASEB J. 11:3:A91. 
Bilous, R.W., Mauer, S.M., Sutherland, E.R., and Steffes, M.W. 1989. Mean glomerular volume and rate of development of diabetic nephropathy. Diabetes 38:1142-1147.

Birkhold, S.G., Morgan, J.B., Miller, R.K., and Sams, A.R. 1992. Proteolytic mechanism of mature cow meat tenderization by calcium injection. Page 153 in: Proceedings of Institute of Food Technologists Annual Meeting, New Orleans, LA.

Bishop, M.L., Duben-Engelkink, J.L., and Fody, E. P. 1992. Clinical chemistry, principles, diabetes. Diabetes 40, 405-412.

Boje, K.M., and Arora, P.K. 1992. Microglial-produced nitric oxide and reactive nitrogen oxides mediate neuronal cell death. Brain Res. 587:250-256.

Booth, A.A., Khalifah, R.G., Todd, P., and Hudson, B.G. 1996. Biochem. Biophys. Res. Commun. 220, 113-119.

Booth, A.A., Khalifah, R. G., Todd, P., and Hudson, B. G. 1997. In vitro kinetic studies of formation of antigenic advanced glycation end products (AGEs): novel inhibition of post-Amadori glycation pathways. J. Biol. Chem. 272, 5430-5437.

Bottje, W.G., and Wideman, R.F., Jr. 1995. Potential role of free radicals in the pathogenesis of pulmonary hypertension syndrome. Poultry and Avian Biology. 6:211-231.

Brandy, B., and Davison, A.J. 1990. Mitochondrial mutations may increase oxidative stress: Implications for carcinogenesis and aging? Free Radic. Biol. Med. 8:523-539.

Braun, E.J., and Dantzler, W.H. 1997. Vertebrate renal system. In: Dantzler, W.H. (Ed) Handbook of Physiology: Comparative Physiology. Vol. 1. pp. 481-576. New York, Oxford University Press.

Brennan, M. 1989. Changes in solubility, nonenzymatic glycation, and fluorescence of collagen in tail tendons from diabetic rats. J Biol. Chem. 35:20947-20952.

Brooks, B.A., Klasing, K.C., and Regenstein, J.M. 1985. Effects of antemortem injected crude papain in chicken muscle. J. Food Sci. 50:1370-1374.

Brownlee, M., Cerami, A., and Vlassara, H. 1988. Advanced products of nonenzymatic glycosylation and the pathogenesis of diabetic vascular disease. Diabetes Metabolism Reviews. 37-451.

Brownlee, M., Pngor, S., and Cerami, A., 1983. Covalent attachment of soluble proteins by nonenzymatically glycosylated collagen. J. Exp. Med. 158:1739-1744. 
Brownlee, M., Vlassara, H., and Cerami, A. 1985. Nonenzymatic glycosylation products on collagen covalently trap lwo-density lipoprotein. Diabetes 34:938-941.

Brownlee, M., Vlassara, H., Kooney, A., Ulrich, P., and Cerami, A. 1986. Aminoguanidine prevents diabetes-induced arterial wall protein cross-linking. Science 232:1629-1632.

Bucala, R., and Cerami, A. 1992. Advanced glycosylation: chemistry, biology, and implications for diabetes andaging. Adv. Pharmacol. 23:1-34.

Buckingham, B., and Reiser, K.M. 1990. Relationship between the content of lyslyl-oxidase dependent crosslinks in skin collagen, nonenzymatic glycosylation and the long-term complications in type I diabetes mellitus. J. Clin. Invest. 86:104-1054.

Bunn, J.F., Higgins, P.J., 1981. Reaction of monosaccharides with proteins; possible evolutionary significance. Science 213:222.

Burrows, W.H., and Quinn, J.P. 1937. The collection of spermatozoa from the domestic fowl and turkey. Poultry Sci. XVI:19-24.

Busse, E.W. 1973. Theory and therapeutics of aging. New York: Medical Press.

Cadenas, S., Rojas, C., Perez-Compo, R., Lopez-Torrez, M., Barja, G. 1994. Caloric and carbohydrate restriction in the kidney: Effects on free radical metabolism. Exp. Gerontol. 29:77-78.

Calkins, E. Aging of cells and people. Clin. Obstet. Gynecol. 1981; 24:165-179.

Cathcart, R., Schwiers, E., Saul, R. Ames, B.N. 1984. Thymine glycol and thymidine glycol in human and rat urine: A possible assay for oxidative DNA damage. Proc. Natl. Acad. Sci. USA 81:5633-5637.

Cefalu, W. T., Bell-Farrow, A. D., Wang, Z., Sonntag,W. E. M., Fu, W.E.,. Baynes, J.W., and Thorpe, S.R. 1995. Caloric restriction decreases age-dependent accumulation of the glycoxidation products, $\mathrm{N}$-(Carboxymethyl) lysine and pentosidine, in rat skin collagen. J. Gerontol: Biol. Sci. 50A:B337-B341.

Cerami, A., Steves, V.J., and Monnier, V.M. 1979. Role of nonenzymatic glycosylation in the development of sequelae of diabetes mellitus. Metabolism 38:431-438.

Cerami, A. 1985. Hypothesis: Glucose as a mediator of aging. J. Am. Geriatr. Soc. 33:626-634.

Cerami, A., Vlassara, J., and Brownlee, M., 1987. Glucose and aging. Science 256: 90-96. 
Ceriello, A., Giugliano, D., Quataro, A., Donzella, C., Dipalo, G. and Lefebvre, P.J. 1991 Vitamin E reduction of protein glycosylation in diabetes. Diabetes Care 14,68-72.

Chandler, M.I., Shannon, W.A., and DeSantis, L. 1984. Prevention of capillary basement membrane thickening in diabetic rats by aldose reductase inhibitors. Invest. Ophthalmol. Visual Sci. 25 (Suppl. ):159.

Chang, K., Uitto, J., Rowold, E.A., Grant, G.A., Kilo, C., and Williamson, J.R., 1980. Increased collagen crosslinkages in experimental diabetes: reversibly $\beta$-aminopropionitrile and Dpenicillamine. Diabetes 29:778-781.

Chaudhry, A.K., Nobubo, M., Reddy, F.R., Yeola, S.N., Morrow, J.D., Blair, I.A., and Marnetti, L. J. 1994. Detection of endogenous malondialdehyde-deoxiguanosine adducts in human liver. Science 265: 1580-1582.

Chen, J.J., Bertrand, J., Yu, B.P. 1995. Inhibition of adenine nucleotide translocase by lipid peroxidation byproducts. Free Radic. Biol. Med. 19:583-590.

chickens. Camp. Biochem. Physiol.. 110C:215-220.

Chipalkatti, S., De, A.K., and Aiyar, A.S. 1983. Effect of diet restriction on some biochemical parameters related to aging in mice. J. Nutr. 113:944-950.

Chou, M.W., Pegram, R.A., Gao, P., and Allaben, W.T., 1991. Effect of caloric restriction on aflatoxin Bl metabolism and DNA modification in Fischer-344 rats. In: Fishbein, L., ed. Biological effects of dietary restriction. Berlin: Springer Verlag ;42-54.

Chung, M.H., Kasai,H., Nishimura, S., Yu, B.P. 1992. Protection of DNA damage by dietary restriction. Free Radic. Biol. Med. 12:523-525.

Comfort, A., 1979. The biology of senescence, 3rd ed. New York: Elsevier.

Conlon, P., Smith, D., and Gowlett, T. 1991. Oxygen radical production by avian leukocytes. Can. J. Vet. Res. 55:193-195.

Corpas, E., Harman, S.M., Blackman, M.R. 1993. Human growth hormone and human aging. Endor. Rev. 14:20-39.

Crijns, R.L., Boudier, H.A.J.S., Wolfenbuttel, B.H.R. 1998 Arteriolar reactivity in conscious diabetic rats: Influence of aminoguanidine treatment. Diabetes; 47:918-923.

Cronlund, A.C. And Woychik, J.H. 1987. Solubilization of collagen in restructured beef with collagenases and alpha-amylase. J. Food Sci. 52:857-860. 
Cutler, R.G. 1984a. Antioxidants, aging, and longevity. In: Pryor, W.A., ed. Free Radicals in Biology. Vol. VI. Orlando: Academic Press. 371-428.

Cutler, R.G. 1984b. Urate and ascorbate: Their possible roles as antioxidants in determining longevity of mammalian species. Arch. Gerontol. Geriatr. 3:321-348.

Danby, R., Bluff, L., Deheny, T.P., and Gibson, W.R. 1982. Effects of alloxan and streptozotocin at high doses on blood glucose levels, glucose tolerance, and responsiveness to sulphonylureas in chickens. Gen. Comp. Endocrinol. 47:159-169.

Davis, P.F., and Stehbens, W.E. 1984. Comparison of nonenzymatic glycosylation of arterial and venous collagen. Biochem. Med. 32:15-22.

Davie, S.J., Gould, B.J. and Yadkin, J.S. 1992. Effect of Vitamin C on glycosylation of protein. Diabetes 41:167-173.

Dean, R.T., Gebricki, J., Gieseg, S., Grant, A. J., and Simpson, J.A. 1992. Hypothesis: A damaging role in aging for reactive protein oxidation products? Mutat. Res. 275:387-393.

Del Maestro, R. F. 1980. An approach to free radical in medicine and biology. Acta Physiol.

Scand. 492:153-168.

Dunn, J.S., Patrick, J. S.,Thrope, S. R., and Bayens, J. W. 1989. Oxidation of glycated protein: age dependent accumulation of $\mathrm{N}$-(Carboxymethyl)lysine in lens proteins. Biochemistry 28:9464-9468.

Dunn, F.A., McCance, D. R., Thorpe, D. R., Lyons, T. J., Baynes, J. W. 1991. Age-dependent accumulation of $\mathrm{N}$-(carboxymethyl) lysine and $\mathrm{N}$-(Carboxymethyl)hydroxylysine in human skin collagen. Biochemistry 30:1205-1210.

Dyer, D.G., Blackledge, J.A., Thorpe, S.R., and Baynes, J.W. 1991a. Formation of pentosidine during nonenzymatic browning of proteins by glucose. J. Biol. Chem. 266:11554-11560.

Dyer, D.G., Blackledge, J.A., Thorpe, S.R., Baynes, J.W. 1991b. Formation of pentosidine during nonenzymatic browning of proteins by glucose: identification of glucose and other carbohydrates as possible precursors of pentosidine in vivo. J. Biol. Chem. 266:11654-11660.

Dyer, D.G., Dunn, J.A., Thorpe, S.R., and Lyons, T.J. 1992. Accumulation of Maillard reaction products in skin collagen in diabetes and aging. Annals of the NY Acad. of Sci. 663:421-422.

Edelstein, D., and M. Brownlee, 1992 a. Mechanistic studies of advanced glycosylation end product inhibition by aminoguanidine. Diabetes 41:26-29. 
Edelstein, D., and M. Brownlee, 1992 b. Aminoguanidine ameliorates albuminuria in diabetic hypertension rats. Diabetologia, 35:96-97.

Eichhorn, G.L. 1979. Aging, genetics and environment. Potential of errors introduced into genetic information transfer by metal ions. Mech. Aging Dev. 9:291-301.

Elgawish, A., Glomb, M., Friedlander, M. and Monnier, V.M. 1996. Involvement of hydrogen peroxide in collagen crosslinking by high glucose in vitro and in vivo. The J. Biol. Chem. 271:12964-12971.

Enesco, J., and Kurk, P. 1981. Dietary restriction reduces fluorescent age-related pigment accumulation in mice. Exp. Gerontology. 16:357-361.

Esterbauer, J., and Schaur, R.J. 1990. Aldehyde formed by lipid peroxidation: Mechanisms of formation, occurrence, and determination. In: Bigo-Pelfrey, C., Ed. Membrane lipid oxidation. Boca Raton, FL: CRC Press :239-268.

Everitt, A.V. 1971. Food intake, growth and the aging of collagen in rat tail tendon. Gerontologia; 17:98-108.

Everitt, A.V., Porter, B.D., and Steele, M. 1981. Dietary, caging and temperature factors in the aging of collagen fibers in rat tail tendon. Gerontology; 27:37-41.

Everitt, A.V., Wyndham, J.R., Barnare, D.L. 1983. The anti-aging action of hypophysectomy in hypothalamic obese rats: effects on collagen aging, age-associated proteinuria development and renal histopathology. Mech. Aging Dev.; 22:233-25.

Eyre, D. R. 1980. Collagen: molecular diversity in the bodys protein scaffold. Science 207:1315-1327.

Farmer, K.J., and Sohal, R.S. 1989. Relationship between superoxide anion radical generation aging in the houseflies, Musca domestica. Free Radic. Biol. Med. 7:23-29.

Foegeding, E.A., and D.K. Larick, 1986. Tenderization of beef with bacterial collagenases. Meat Sci. 18:201-214.

Fontana, E.A., Weaver, Jr., W.D., Watkins, B.A. and Denbow, D.M. 1992. Effect of early feed restriction on growth, feed conversion and mortality in broiler chickens. Poultry Sci. 72: 243-250.

Francis, M.J.O., Ellis, J.P., and Hockaday, T.D.R. 1974. Skin collagen in diabetes in relation to treatment. Proc. R Soc. Med. 67:35-36. 
Freeman, B.A. 1984. Biological sites and mechanisms of free radical production. In: Armstrong, D., Sohal, R., Cutler, R.G., Slater, T., eds. Free radicals in molecular biology, aging, and disease. New York: Raven Press :43-52.

Frei, B. 1994. Reactive oxygen species and antioxidant vitamins: Mechanisms of action, Am. J. Med. 97:5.

Fu, M.-X., Knecht, K. J., Thrope, S.R., Baynes, J. W. 1992. Role of oxygen in crosslinking and chemical modification of collagen by glucose. Diabetes 41 (Suppl. 2):42-48.

Fu, M.X., Thorpe, S.R., and Baynes, F.W. 1994a. Effects of aspirin on glycation, glycosylation and crosslinking of collagen. In: Maillard Reactions in Chemistry, Food and Health. Labuza T.P., Reineccius, G.A., Monnier, V.M., O’Brien, J.O., Byenes, J.W. Eds. Royal Society of Chemistry, Cambridge, U.K. p 95-100.

Fu, M-X, Wells-Knect, K.J., Blackledge, J.A., Lyons, T.J., Thorpe, S.R., and Baynes, J.W. 1994b. Glycation, glycoxidation and crosslinking of collagen by glucose: kinetics, mechanisms, and inhibition of late stages of the Maillard reaction. Diabetes 43:676-683.

Fujimoto, D. 1977. Isolation and characterization of a fluorescent material in bovine Achilles tendon collagen. Biochem Biopsy Res connum 76:1124-1129.

Fujimoto, D. 1982. Aging and crosslinking in human aorta. Biochem. Biophys Res commun. 109:1264-1269.

Fujimoto, D. 1988. Crosslinking of collagen. In: Klignan, A.M., Takase, Y., eds. Cutaneous aging. Tokyo: U. Tokyo Press:263-274.

Galeski, A., Kastelic, J, Baer, E., and Kohn, R.R. 1977. Mechanical and structural changes in rat tail tendon induced by alloxan diabetes and aging. J. Biomech 10:775-782.

Glomb, M.A., and Monnier, V.M. 1995. Mechanism of protein modification by glyoxal and glycolaldehyde: reactive intermediates on the Maillard reaction J Biol Chem 270: 10017-10026.

Golub, L.M., Greenwald, R.A., Zebrwoski, E.J., and Ramamurthy, N.S. 1978. The effect of experimental diabetes on the molecular characteristic of soluble rat-tail tendon collagen. Biochem Biophys Acta 534:73-81.

Grandhee, S.K., and Monnier, V.M. 1991. Mechanisms of formation of the Maillard protein cross-link pentosidine: ribose, glucose, fructose and ascorbate as pentosidine precursors. J. Biol. Chem. 266:11649-53. 
Greshaman, R., Gilbert, D.L., Nye, S.W., Dwyer, P., and Fenn, W.O. 1954. Oxygen poisoning and x-irradiation: A mechanism in common. Science 119; 623-6261.

Grgic, A., Rosenbloom, A.L., Weber, F.T, and Giordano, B. 1975. Joint contract in childhood diabetes. N. Engl. J Med. 292:372-379.

Grinna, L.S. 1977. Changes in cell membrane during aging. Gerontology 23:452-464.

Guarnieri, C., Muscari, C., and Caldarera, C.M. 1993. Mitochondrial production of oxygen free radicals in the heart muscle during the life-span of the rat: peak at middle age. Experiencia Suppl.

$62: 47$.

Guitton, J.D., Le pape, A., Sizaret, P.Y., and Muh, J.P. 1981. Influences of in vitro nonenzymatic glycosylation of type I collagen fibrillogenesis. Biosci. Rep. 1;945-954.

Guitton, J.D., Le Pape, A., and Muh, J.P. 1984. Influences of in vitro non-enzymatic glycosylation on the physicochemical parameters of Type I collagen. Collagen Relat. Res. 4:253-264.

Hadley, E.C. 1993. Morbid consequences of aging processes: mythodologic issues. In: Musculoskeletal soft-tissue Aging: Impact on Mobility. Buckwalter, J.A., Goldberg, B.M., Woo SL-y, Eds. Rosemont IL, American Academy of Orthopedic Surgeons, p.35-47.

Hamlin, C.R., Kohn, R.R., and Luschin, J.H. 1975. Apparent accelerated aging of human collagen in diabetes mellitus. Diabetes 24:902-904.

Hammes, H.P., Martin, S. Federlin, K., Geisen, K., and Brownlee, M. 1991. Aminoguanidine treatment inhibits the development of experimental diabetic retinopathy. Proc Natl Acad Sci USA 88: 11555-11558.

Hansford, R.F. 1983. Bioenergetics in aging. Biochem. Biophys. Acta 726:41-80.

Harrison, D.E., and Archer, J. R. 1978. Measurement of changes in mouse tail collagen with age: Temperature dependence and procedural details. Exp. Gerontol. 13:75-82;

Harrison, D.E.; and Archer, J. R. 1983. Physiological assays for biological age in mice: Relationship of collagen, renal function, and longevity. Exp. Aging Res.; 9:245-251.

Harrison, D.E., Archer, J.R., Sacher, G.A., and Boyce, F.M. 1992. Tail collagen aging in mice of thirteen different genotypes and two species: Relationship to biological age. Exp. Gerontol. 1978; 13:63-73. 
Harman, D. 1956. Aging: A theory based on free radical and radiation chemistry. J. Gerontol. 11:298-300.

Hata, F., Igaki, N., Nakamiki, T., Masuda, S., Nishimoto, S., Oimomi, M., Baba, S., and Kato, H. 1988. Suppressive effect of $\propto$-ketoaldehyde dehydrogenase on the advanced process of the Maillard reaction. Diabetes Res. Clin. Pract. 5:5413.

Haut, R.C. 1986. The influence of specimen length on the tensile failure properties of tendon collagen. J. Biomechanics 19:951-955.

Hayase, F., Kim, Y.H., and Kato, H. 1991. Inhibition of the protein crosslinking in a Maillard reaction by aminoguanidine and Semicarbazide. Agric. Biol. Chem. 55:1435-1436.

Hazelwood, R.L. 1986. Carbohydrate metabolism. In: Sturkie, P.D., Avian Physiology. 4th ed. Springer-Verlag, New York. p. 303-325.

Hazelwood, R.L., and Lorenz, F. 1995. Effects of fasting and insulin on carbohydrate metabolism of the domestic fowl. Am. J. Physiol. 197:47-51.

Hegner, D. 1980. Age-dependence on molecular and functional changes in biological membrane properties. Mech. Ageing Dev. 14: 101-118.

Heller, D.A., and McClearn, G.E. 1992. A longitudinal genetic study of tail tendon fibre break time. Age Ageing 21:129-134.

Hellerstrom, C., Anderson, A., Sandler, S., and Swenne, I. 1986. Mechanisms of destruction and repair of the pancreatic-cell. Transpl. Proc. 18:1509-1512.

Hellsten, Y., Tullson, P.C., Richter, E.A., and Bangsbo, J. 1997. Oxidation of urate in human skeletal muscle during exercise. Free Radic. Biol. and Med. 22:169-174.

Hempe, J. M., Lauxen, R. C., and Savage, J. E. 1988. Rapid determination of egg weight and specific gravity using a computerized data collection system. Poultry Sci. 67:902-907.

Higgins, K.A., Stout, J.T., Heller, D.A., Parker, R.F. 1991. Individual variability in tail tendon fiber break time in three age cohorts of different strains of mice. Exp. Gerontol.; 26:467-477.

Hirsch, J., Petrakova, E., Feather, M.S., and Bransens, C.L. 1995. Carbohydr. Res. 267:17-25.

Holehan, A.M., and Merry, B.J. 1986. The experimental manipulation of aging by diet. Biol. Rev. 61:329-368. 
Holliday, R. 1992. The ancient origins and causes of ageing. News Physiol. Sci. 7:34-40.

Holmes, J. D., and S. N. Austad, 1995. Birds as animal models for the comparative Biology: prospectus. J. Geront: Biol. Sci. 50A:B59-B66.

Horton, A.A., and Fairhurst, S., 1987. Lipid peroxidation and mechanisms of toxicity. In CRC Critical Reviews in Toxicology :27.

Hunt, J.V., Bottoms, M.A., and Mitchinson, M.J. 1993. Oxidative alterations in the experimental glycation model of diabetes mellitus are due to protein-glucose adduct oxidation. Biochem $\mathbf{J}$ 291:529-535.

Iqbal, M., Probert, L.L., and Klandorf, H. 1997. Effect of dietary aminoguanidine on tissue pentosidine and reproductive performance in broiler breeder hens. Poultry Sci. 76:1574-1579.

Iqbal, M., Probert, L.L., Al-humadi, N.H. and Klandorf, H. 1998 a. Protein glycosylation and advanced glycosylation endproducts (AGEs): An avian solution. J. Gerontol: Biol. Sci. In review.

Iqbal, M., Kenney, P.B., Klandorf, H. 1998 b. Age-related changes in meat tenderness and tissue pentosidine: Effect of diet restriction and aminoguanidine in broiler breeder hens. Poultry Sci. In review.

James, V.J, Delbridge, L., McLennan, S.V, and Yue, D.K. 1991. Use of X-rasy diffraction in study of human diabetic and aging collagen. Diabetes 40:391-394.

Jiang, Z.Y., Woollard, A.C.S., and Wolff, S.P. 990. Hydrogen peroxide production during experimental protein glycation. FEBS Lett 69-71.

Junod, A., Lambert, A., Stauffacher, W., and Renold, A. 1969. Diabetogenic action of streptozotocin: relationship of dose to metabolic response. J. Clin. Invest. 48:2129.

Kaiser, F. E., and S. G. Korenman, 1988. Impotence in diabetic men. Amer. J. Med. 85:147.

Kawada, J., Toide, J., Nishida, M., Yoshimura, Y., and Tsujihara, K. 1986. New diabetogenic streptozotocin analogue, 3-O-methyl-2\{[(methylnitrosoamino) carbonyl $]$ amino $\}$-Dglucopyranose: Evidence for a glucose recognition site on pancreatic-cells. Diabetes. 35:74.

Kehrer, J. P., and Smith, C.V. 1994. Free radical in biology: Source, reactivities, and role in the etiology of human diseases. In: Frie, B., ed. Natural antioxidants in human healths and disease. San Diego: Academic Press :25-62.

Kenney, P.B., Slider, S.D. Nayak, R.R. and Massey, J.W. 1996. Stunning method and time held 
in a transport coop affect $\mathrm{pH}$ decline and muscle quality in broilers. Poultry Sci. 75: (Suppl.1-72):55.

Kent M. J.C., Light, N.D., and Bailey, A.J. 1985. Evidence for glucose-mediated covalent crosslinking of collagen after glycosylation in vitro. Biochem J 225-: 545-52.

Khalifah, R.G., Todd, P., Booth, A.A., Yang, S.X., Mott, J.D., and Hudson, B.G. 1996. Biochemistry 35, 4645-4654.

Khatan, M., Soldan, Z., David, I., Li, W. and Rockey, J.H. 1988. Inhibitory effects of pyridoxal phosphate, ascorbate and aminoguanidine on nonenzymatic glycosylation. Life Sci. 43:1725-1731.

Kochakian, M., Manjula, B.N., and Egan, J.J. 1998. Chronic dosing with aminoguanidine and novel advanced glycosylation endproduct-formation inhibitors ameliorates crosslinking of tail tendon collagen in STZ-induced diabetic rats. Diabetes 45:1694-1996.

Kohn R.R., Cerami, A., and Monnier, V.M. 1984. Collagen aging in vitro by nonenzymatic glycosylation and browning. Diabetes 33:57-59.

Khatami, M., Suldan, A., David, I., Li., W., and Rockey, J.H. 1988. Life Sci. 43:1725-1731.

Kilo, C. Volger, N., Williamson, J.R. 1972. Muscle capillary basement membrane charges related to aging and to diabetes mellitus. Diabetes 2:881-905.

Klandorf, H., Holt, S., McGowan, J., Pinchasov, Y., Deyette, D., and Peterson, R. 1995. Hyperglycemia and nonenzymatic glycation of serum and tissue proteins in chickens. Comp Biochem. Physiol. 110C/2:215-220.

Klandorf, H., Zhou, Q., and Sams, A.R. 1996. Inhibition by aminoguanidine of glucose-derived collagen crosslinking in skeletal muscle of broiler breeder hens. Poultry Sci. 75:432-437.

Klandorf, H., Porbert, L.L., and Iqbal, M. 1998. In the defense against hyperglycemia: Avian strategy. Br. Poutry Sci. In review.

Klein, L., Butcher, M.A., Sudilovsky, O., Kikkawa, R., and Miller, M. 1975. Quantification of collagen from human nondiabetic and diabetic kidneys. Diabetes 24:1057-1065.

Kohn, R.R. 1983. Effects of age and diabetes mellitus on cyanogen bromide digestion of human dura mater collagen. Connect Tissue Res. 11:169-173.

Kohn, R.R., and Schnider, S.L. 1982. Glycosylation of human collagen. Diabetes 31:47-51. 
Kohn, R.R., Cerami, A., and Monnier, V. 1984. Collagen aging in vitro by nonenzymatic glycosylation and browning. Diabetes 33:57-59.

Koizumi, A., Weindruch, R., Walford, R.L. 1987. Influences of dietary restriction and age on liver enzyme activities and lipid peroxidation on mice. J. Nutr. 117:361-367.

Koizumi, A., Wada, Y., Tsukada, M., and Hasegawa, J. 1989. Low blood glucose levels and small Islets of Langerhans in the pancreas of caloric-restricted mice. Age 12:93-96.

Kondaiah, N., and Panda, B. 1992. Processing and utilization of spent hens. Worlds Poult. Sci. $56: 255-265$.

Koohmaraie, M., Seideman, S. C., Schollmeyer, J. E., Dutson, T. R., and Crouse, J. D. 1987. Effect of post-mortem storage on $\mathrm{Ca}^{+2}$-dependent proteases, their inhibitor and myofibril fragmentation. Meat Sci. 19:187-196.

Koohmaraie, M., Babiker, A. S., Schroeder, A. L. Merkel, , R. A., and Dutson, T. R. 1988. Acceleration of postmortem tenderization in ovine carcasses through activation of $\mathrm{Ca}^{+2}$ dependent proteases. J. Food Sci. 53:1638-1641.

Koohmaraie, M., Crouse, J. D., and Mersman, J. J. 1989. Acceleration of postmortem tenderization in ovine carcasses through infusion of calcium chloride: Effect of concentration and ionic strength. J. Anim. Sci. 67:934-942.

Koohmarie, M., Whipple, G., and Crouse, J. D. 1990. Acceleration of postmortem tenderization in lamb and Barham-cross beef carcasses through infusion of calcium chloride. J. Anim. Sci. 68:1278-1283.

Kowland, A., and Kirkwood, T.B.L. 1994. Towards a network theory of aging: A model combining the free radical theory and protein error theory. J. Thor. Biol. 168:75-94.

Kristal, B., and Yu, B. 1992. An emerging hypothesis: Synergistic induction of aging by free radicals and Maillard reactions. J. Gerontology: Biol. Sci. 47:B107-B114.

Kristal, B.S., Park, B.K., and Yu, B.P. 1996. 4-Hydroxyhexenal is a potent inducer of the mitochondrial permeability transition. J Biol. Chem. 271:6033-6038.

Kristein, M., Brett, J., Rodoff, S., Stern, D. and Vlassara, J. 1990. Advanced glycosylation endproducts selectively induce monocyte migration across intact endothelial cell monlayers, and elaboration of growth factors: role in aging and diabetic vasculopathy. Proc. Natl. Acd. Sci. USA 87:9010-9014. 
Kirstein, M., Aston, C., Hintz, R., and Vlassara, H. 1991. Receptor-specific induction of insulinlike growth factor I (IGF-I) in human monocytes by advanced glycoslation endproduct-modified proteins. J Clin. Invest. 90:439-446.

$\mathrm{Ku}, \mathrm{H}$., and Sohal, R.S. 1993. Comparison of mitochondrial prooxidant generation and antioxidant defenses between rat and pigeon: possible basis of variation in longevity and metabolic potential. Mech. Aging Dev. 72:67-76.

Kumari., K., Umar, S., Bansal, B., and Sahib, M.K. 1991. Inhibition of diabetes-associated complications by nucleophilic compound. Diabetes 40:1079-1084.

Laganiere, S., and Yu, B.P. 1993. Modulation of membrane phospholipid fatty acid composition by age and food restriction. Gerontology 39:7-18.

Laleski, A., Kastelic, J., Baer, E., and Kohn, R.R. 1977. Mechanical and structural changes in rat tail tendon induced by alloxan diabetes and aging. J Biomech; 10:775-782.

Le Pape, A., Guitton, J.D., Muh, J.P. 1984. Distribution of non-enzymatically bound glucose in vivo and in vitro glycosylated Type I collagen molecules. FEBS Lett.170:23-27.

Lerner, L. P., French, N., McIntyre, D., and Baxter-Jones, C. 1993. Age-related changes in egg production, fertility, embryonic mortality, and hatchability in commercial turkey flocks. Poultry Sci. 72:1025-1039.

Lindstedt, S. L., and Calde, W. A. 1976. Body sized and longevity in Birds. Condor 78,91-94.

Lyons T.J., and Kennedy, L., 1985. Effect of in vitro nonenzymatic glycoslation of human skin collagen on susceptibility to collagenase digestion. Eur J Cain Invest 15: 128-131.

Lyons, T.J., Bailie, D.E., Dyer, D.G., Dunn, J.A., and Baynes, J.W. 1991. Decrease in skin collagen glycation with improve glycemic control in patients with insulin-dependent diabetes mellitus. J Clin. Invest. 87:1910-1915.

Madia A.A., Roxovski, S.J., Kagan, H.M., 1979. Cahgnes in lung lysyl oxidase activity in streptozotocin-diabetes and in starvation. Biochem. Biophys Acta 585:481-487.

Maekawa, T., Ratinasamy, K. I., Altman, Y. K., and Forbes., W. F. 1970. Changes in collagen with age-I. The extraction of acid soluble collagens from skin of mice. Exp. Geront. 5:177-186.

Masoro, E.J. 1988. Food restriction in rodents: An evaluations of its role in the study of aging. J Gerontol. Biol. Sci. 43:B59-B64. 
Masoro, E. J., Katz, M.S., Mcmahan, C.A. 1989. Evidence for the glycation hypothesis of aging from the food restricted rodent model. J. Gerontol. 44:B20-B22.

Masoro, E.J., and McCarter, R.J.M. 1991. Aging as a consequence of fuel utilization. Aging Clin. Exp. Res. 3:117-128.

Masters, B.S.S. 1994. Nitric oxide syntheses: Why so complex? Annu. Rev. Nutr. 14:131-145.

Mathieu, C., M. Waer, K. Casteels, J. Laureys, and R. Bouillon, 1995. Prevention of Type I diabetes in NOD mice by non-hypercalcemic doses of a new structural analog of 1, 25dihydroxyvitamin $\mathrm{D}_{3}$, DH 1060. Endocrinology 136:866-872.

Matsuo, M.O. 1986. Age-related alterations in antioxidant defense, in: Free Radicals in Aging, Meadows, J., Smith, R.C., Reeves, J. Uric acid protects membranes and linolenic acid from ozone-induced oxidation. Biochem. Biophys. Res. Commun. 137:536-541.

Medvedev, Z. 1990. An attempt at rational classification $f$ theories of aging. Biol. Rev. 65: 375-398.

Menzel, E.J., and Reihsner, R. 1991. Alterations of biochemical and biomechanical properties of rat tail tendon caused by nonenzymatic glycation and their inhibition by dibasic amino acid arginine and lysine. Diabtologia 34:12-16.

Miksik, I, Struzinsky, R., and Deyl, Z. 1991. Change with age of UV absorbance and fluorescence of collagen and accumulation of epsilon-hexosyllysine in collagen from Wistar Rats living on different food restriction regimes. Mech Ageing Dev 57:163-174.

Miksik, I., and Deyl, Z. 1991. Change in the amount of epsilon-hexoyllysine, UV absorbance, and fluorescence of collagen with age in different animal species. J Gerontol 46:B111-B116.

Minaker K.L. 1987. Aging and diabetes mellitus are risk factors for vascular disease. Am. J Med. 82 (Suppl. 1B):47-53.

Moncada, S., Palmer, R.M.J., Higgs, E.A. 1992. Nitric oxide: Physiology, pathology, and pharmacology. Pharm. Rev. 43: 109142.

Monnier, V.M, and Cerami, A. 1981. Nonenzymatic browning in vivo: possible process for aging of long lived proteins. Science 211:493.

Monnier, V.M., Kohn, R.R., and Cerami, A., 1984. Accelerated age-related browning of human collagen in diabetes mellitus. Proc. Nalt Acad Sci USA 81:583-587. 
Monnier, V.M., Vishwanath, V., Frank, K.E., Elmets, C.A., Dauchot, P., and Kohn, P.R. 1986. Relationship between complications of type I diabetes mellitus and collagen-linked fluorescence. N. Engl. J. Med. 314:403-408.

Monnier, V.M. 1989. Toward a Maillard reaction theory of aging. In: The Maillard Reaction in Aging, diabetes and Nutrition, Prog. Clin. Biol. Res., Baynes, J.W. and Monnier, V.M., Eds., Alan R. Liss, New York.

Monnier, V.M., Sell, D.R., Abdul-Karim, F.W., and Emancipator, S.N. 1988. Collagen browning and crosslinking are increased in chronic experimental hyperglycemia. Diabetes 37:867-872.

Monnier, V. M. 1989. Toward a Maillard reaction theory of aging. Prog. Clin. Biol. Res. 304:1-22.

Monnier, V. M. 1990. Nonenzymatic glycosylation, the Maillard reaction and the aging process. J. Gerontol: Biol. Sci. 45, B105-B111.

Monnier, V.M., Sell, D.R., Nagaraj, R.H., Miyata, S. 1991. Mechanisms of protection against damage mediated by the Maillard reaction in aging. Gerontology 37:152-165.

Monnier, V., Glomb, M., Elgawish, A., and Sell, D. 1996 The mechanism of collagen crosslinking in diabetes: A puzzle nearing resolution. Diabetes 45:S67-S72.

Morgan, J., R. K. Miller, F. M. Mendez, D. S. Hale, and J. W. Savell, 1991. Using calcium chloride injection to improve tenderness of beef from mature cows. J. Anim. Sci. 69:4469 -4476.

Mote, P.L., Grizzle, J. M, Walford, R.L., and Spindler, S.R. 1991. Influences of age and calorie restriction expression of hepatic genes for xenobiotic and oxygen metabolizing enzymes in the mouse. J. Gerontol. 46: B95-B100.

Moultrie, F. 1956. A new technique for the artificial insemination of caged hens. Poultry Sci. $35: 1230-1234$.

Mullarkey, C.J., Edelstein, D., and Brownlee, M. 1990. Free radical generation by early glycation products: a mechanism for accelerated atherogenesis in diabetes. Biochem. Biophys. Res. commun. 173:932-939.

Nagaraj, R.H., Timothy, T.S., Sell, D.R., Fogarty, J., Engerman, R.L., and Monnier, V.M. 1996. Evidence of a glycemic threshold for the formation of pentosidine in diabetic dog lens but not in collagen. Diabetes 45:587-594.

Nakamura, R., Sekoguchi, S., and Sato, Y. 1975. The contribution of intramuscular collagen to the tenderness of meat from chickens with different ages. Poultry Sci. 54:1604-1612. 
Namiki, M., and Hayashi, T. 1975. Development of novel free radicals during the animo-carbonyl reaction of sugars with amino acids. J Agric. Food Chem. 23:487-491.

Narayanan, A.S., Sandberg, L.B., Jones, K., Coleman, S.S., and Bagley, R.A. 1982. Lysyl oxidase activities of male and female turkey aorta. Exp. Mol Pathol. 36:107-117.

Nohl, H. and Hegner, D. 1990. Do mitochondria produce oxygen radicals in vivo? Eur. J. Biochem. 82:563.

Nolen, G.A. 1972. Effect of various restricted dietary regimens on the growth, health, and longevity of albino rats. J. Nutr. 102:1477-1494.

Nurmahmudi and Sams, A. R. 1997. Tenderizing spent fowl meat with calcium chloride. 1. Effect of delivery method and tumbling. Poultry Sci. 76:534-537.

Odetti, P. R., Borgoglio, A., Pascale, A.D., Polandi, R. and Adezati, L. 1990. Prevention of diabetes-increased aging effect on rat collagen-linked fluorescence by aminoguanidine and rutin. Diabetes. 39:796-801.

Oimoni, M., Kitamura, Y., Nishimoto, S., Matsumoto, S., Hatanaka, H., and Baba, S. 1981. Age-related acceleration of glycation of tissue proteins in rats. J. Gerontol. 421:695-698.

Oldham, K.T., Guice, K.S., Ward, P.A., and Johnson, K.J. 1988.The role of oxygen radicals in immune complex injury. J. Free Radic. Biol. Med., 4:387.

Orr, W.C., and Sohal, R.S. 1994. Extension of life-span by over expression of superoxide dismutase and catalase in Drosophila melanogaster. Science 263:1128-1130.

Oxuland, H. and Andreassen, T. 1992. Aminoguanidine treatment reduces increase in collagen stability of rats with experimental diabetes mellitus. Diabetologia 35a:19-25.

Pahlavani, M.A., Haley-Zitlin, B.V., Richardson, A. 1994. Influence of dietary restriction on gene expression: Changes in transcription of specific genes. In; Yu, B.P. ed. Modulation of aging processes by dietary restriction. Boca Raton, FL: CRC Press:143-156.

Ou, R., and Wolff, P. 1993. Aminoguanidine: A drug proposed for prophylex in diabetes inhibits catalase and generates hydrogen peroxide in vitro. Biochem. Parmacol. 46:1139-1144.

Papa, C. M., Young, L.L., and Lyon, C.E. 1989. Calcium-induced tenderization of early-deboned breast fillets. Poultry Sci. 68:110.

Parmley, L., Mufti, A.G., and Downey, J.M. 1992. Allopurinol therapy of ischemic heart disease with infarct extension. Can. J. Cardiol. 8:280-286. 
Paul, R.G. and Bailey, A.J. 1996. Glycation of collagen: the basis of its central role in the late complications of aging and diabetes. Int. J. Biochem. Cell. Biol. 28:1297-1310.

Perejda, A.J., and Uitto, J. 1982. Nonenzymatic glycosylation of collagen and other protiens: Relationship to development of diabetic complications. Collagen Relata. Res. 2:81-88.

Perejda A.J., Zaragoza, E.J, Eriksen, E., and Uitto, J. 1984. Nonenzymatic glycosylation of lysyl and hydroxylysyl residues in type I and Type II collagen. Collagen Relat. Res 4:427-439.

Pierrefiche, G., and Laborit, H. 1995. Oxygen free radicals, melatonin, and aging. Experimental Gerontology. 30:3/4:213-227.

Pillsbury, H.C., Hung, W., Kyle, M.C., and Freis, E.D. 1974. Arterial pulse wave velocity and systolic time intervals in diabetic children. Am Heart J 87:783-790.

Porta, E.A. 1988. Role of oxidative damage in the aging process. In: Chow, C.K., ed. Antioxidant defense mechanisms, vol. 3. Boca Raton, FL: CRC Press :1-52.

Ramamurthy, N.S., Zebrowski, E.J., and Golub, L.M. 1972. The effects of alloxan diabetes on gingival collagen metabolism in rats. Arch Oral Biol 17:1551-1560.

Regan, T.J., Lyons, M.M., Ahmed,S.S., Levinson, G.E, Oldewurtel, H.A., Ahmed, M.R., and Haider, B. 1977. Evidence for cardiomyopathy in familial diabetes mellitus. J. Clin Invest 60:885-899.

Rehkter, M.D., Yhang, K., Naryana, A.S., Phan, S., Schork, M.A. and Gordon, D. 1993. Type I collagen expression in human atherosclerosis. Am. J pathol. 143:1634-1648.

Reiser, K.M. 1991. Nonenzymatic glycation of collagen in aging and diabetes. Proc. Soc. Exp. Biol. Medl 196: 17-29.

Reiser, K. M., 1991 Nonenzymatic glycation of collagen in aging and diabetes. Prec. Soc. Exp. Biol. Med. 196:17-29.

Reiser, K.M., McCormick, R. and Rucker, R.B. 1992. Crosslinking in collagen and elastin. FASEB J. 6:2439-2449.

Reiser, K.M. 1994. Influence of age and long-term dietary restriction on enzymatically mediated crosslinks and nonenzymatic glycation of collagen in mice. J. Gerontol: Biol. Sci. 49:B71-B79.

Richard, S., Tamas, C., Sell, D.R., Monnier, V.M. 1991. Tissue-specific effects of aldose reductase inhibition on fluorescence and crosslinking of extracellular matrix in chronic galactosemia. Relationship to pentosidine crosslinks. Diabetes 40:1049-1056. 
Rendell, M., Stephen, P.M., Paulsen, R., Valentine, J.L., Rasbold, K., Hestorff, T., Eastberg, S., and Shint, D. 1985. An interspecies comparison of normal levels of glycosylated hemoglobin and glycosylated albumin. Comp. Biochem. and Physiol. 81B:819-822.

Robertson, J., Ratcliff, D., Bouton, P.E., Harris, P.V. and. Shorthose, W.R. 1984. Effect of cooking temperature and animal age on the shear properties of beef and buffalo meat. J. Food Sci. 49:1163-1166.

Robins, S.P., and Bailey, A.J. 1972. Age related changes in collagen: The identification of reducible lysine-carbohydrate concentration products. Biochem Biopshys Res Commum 48:76-84.

Robins, S.P. 1982. Analysis of the crosslinking components in collagen and elastin. Meth. Biochi. Analysis 28:330-379.

Robinson, F.E., Hardin, R.T. and Williams, B.J. 1991. The influence of egg sequence position on fertility, embryo viability, and embryo weight in broiler breeders. Poultry Sci. 70 :760-765.

Robison, W.G., Kador, P.F., and Kinoshita, J.H. 1983. Retinal capillaries: Basement membrane thickening by galactosemia prevented with aldose reductase inhibitor. Science 221:1177-1179.

Roch-Ramel, F. and Peters, G. 1978. Urinary excretion in nonhuman mammalian species. In: Kelley, W. N.; Weiner, I. M. eds. Uric acid, Handbook of Experimental Pharmacology. New York: Springer; 211-225.

Rodnan, G.P., Lipinsky, E., and Luksick, J. 1979. Skin thickness and collagen content in progressive systemic sclerosis and localized scleroderma. Arthritis Rheum 22:130-140, 1979.

Rogozinski, S., Blumenfeld, O., and Seifter, S. 1992. The nonenzymatic glycosylation of collagen. Arch Biochem Biophys 221;428-437.

Rosenthal, M., and Olenick, L.J. 1988. Evaluation of a single color reading method for determining fructosamine. Clin. Chem. 34:360-363.

Rossini, A.A., and Dulin, W.E. 1976. Studies of streptozotocin enamors on pancreatic beta cell toxicity. Diabetes. 35:384.

Sabatino, F., Masoro, E.J., McMahan, A., and Kuhn, R.W. 1991. Assessment of the role of the glucocorticoid system in aging processes and in the action of food restriction. J. Gerontol. Biol. Sci. 46:B171-179.

Sapolsky, R.W., Krey, L., and McEwen, B.S. 1986. The neuroendocrinology of stress and aging: 
Glucocorticoid cascade hypothesis. Endocr. Rev. 7:284-301.

SAS Institute, 1990. SAT/STAT7 Users Guide: Statistics. Release 6.04, SAS Institute Inc., Cary, NC.

Saul, R.L., Gee, P., and Ames, B.N. 1987. Free radicals, DNA damage, and aging. In: Warner, J. R., Butler, R.N., Sprott, R.L., Schneider, E. L., eds. Modern biological theories of aging. New York: Raven Press :113-129.

Scheynius, A., and Jedal, I.B. 1971. On the mechanism of glucose protection against alloxan toxicity. Diabetologia. 7:252-255.

Schnider, S.L., and Kohn, R.R. 1982. Effects of age and diabetes mellitus on the solubility of collagen from human skin, tracheal cartilage and dura mater. Exp Gerontol. 17:185-194.

Schreiber, G., Tiemeyer, W., Flurer, C.I., and Zucker, H. 1986. Purine metabolites in serum of higher primates, including man. Int. J. Primatol. 7:521-531

Schuyler, M.R., Niewohner, D., Inkley, S.R., and Kohn, R.R. 1976. Abnormal lung elasticity in juvenile diabetes mellitus. Am. Rev. Respir. Dis. 13:37-41.

Schweiger, F.F., Uehlein-harrell, S., Von Hegel, G., and Wiesner, H. 1991. Vitamin A (retinol and retinyl esters), $\alpha$-tocopherol and lipid levels in plasma of captive wild mammals and birds. Xentralblatt fur Veterinarmedizin-Reihe. A38:35-42.

Sell, D.R. and Monnier, V.M. 1989. Structure elucidation of a senescence crosslink from human extracellular matrix: Implication of pentoses in the aging process. J. Biol. Chem. 264:21597-21602.

Sell, D.R., and Monnier, V.M. 1990. End-stage renal disease and diabetes catalyze the formation of a pentose-derived crosslink from aging human collagen. J. Clin. Invest. 85:380-384.

Sell, D.R., Lapolla, A., Odetti, P., Fogarty, J,. and Monnier, V.M. 1992. Pentosidine formation in skin correlates with severity of complications in individuals with long-standing IDDM. Diabetes, 41:1286-1291.

Sell, D.R., Lane, M.A., Johnson, W.A., Masoro, E.J., Mock, O.B., Reiser, K.M., Fogarty, J.F., Cutler, R.G., Ingram, D.K., Roth, G.S., and Monnier, V.M. 1996. Longevity and the genetic determination of collagen glycoxidation kinetics in mammalian senescence. Proc. Natl. Acad. Sci. 93:485-490. 
Sell, R. And Monnier, V. M. 1997. Age-related association of tail tendon break time with tissue pentosidine in DBA/2 vs C57BL/6 mice: the effect of dietary restriction. J. Gerontol: Biol. Sci. 52A:B277-B284.

Sevanian, A., Davies, K.J.A., and Hochstein, P. 1991. Serum urate as an antioxidant for ascorbic acid. Am. J. Clin. Nutr. 54:1129S-1143S.

Shapiro, F. Mahagna, M. and Nir, I. 1997. Stunting syndrome in broilers: Effect of glucose or maltose supplementation on digestive organs, intestinal disaccharidase, and some blood metabolites. Poultry Sci 76,369-380.

Shimokawa, I., Yu, B.P., Higani, Y., Ikeda, T., and Masoro, E.J. 1993. Dietary restriction retards onset but not progression of leukemia in male F344 rats. J. Gerontol: Biol. Sci. 48:868-B73.

Shimokomaki, M., Elsden, D. F. and Bailey, A. J. 1972. Meat tenderness: Age-related changes in bovine intramuscular collagen. J. Food Sci. 37:892.

Short, R., Williams, D.D., and Bowden, D.M. 1997. Circulating antioxidants as determinants of the rate of biological aging in Pigtailed Macaques (Macaca nemestrina). J Gerontol.: Biol. Sci. 52A:B26-B30.

Siegel, R.C. 1979. Lyslyl oxidase. Int. Rev. Connect. Tissue Res. 8:73-118.

Sies, H. 1985. Oxidative stress: Introductory remarks. In: Sies, H., ed. Oxidative stress. New York: Academic Press:1-8.

Simon, H., and Dubois, M.P. 1980. Failure of a sublethal streptozotocin dose to induce diabetes in the chicken. Horm. Metab. Res. 12:631-632.

Sims, T.J., And Bailey, A.J. 1982. The structure and growth of muscle. Pages 27-47 in: Meat Science. R. A. Lawrie. ed. 5th, New York.

Sims, T.J., and Bailey, A.J. 1992. Quantitative analysis of collagen and elastin crosslinks using a single column system. J. Chromatog 582: 49-55.

Sims, T.J., Rasmussen, L.M., Oxlund, H., and Bailey, A.J. 1996. The role of glycation crosslinks in diabetic vascular stiffening. Diabetologia 39: 946-951.

Sohal, R.S., and Allen R.G. 1990. Oxidative stress as a causal factor in differentiations and aging: A unifying hypothesis. Exp. Gerontol. 25, 499-522.

Sohal, R.S., Agrawal, S., Dubey, A., and Orr, W.C. 1993. Protein oxidative damage is associated with life expectancy of houseflies. Proc. Natl. Acad, Sci. USA 90:7255-7259. 
Sohal, R.S., Ku, H.H., Agrawal, S., Forster, M.J., and Lal, H. 1994. Oxidative damage, mitochondrial oxidant generation and antioxidant defense during aging and in response to food restriction in the mouse, Mech. Ageing Dev. 74:121-133.

Sohal, R. S., and Orr, W. C. 1995. In molecular aspect of aging, K. Esser and G. M. Martin, Eds (wiley NY) p.109-127.

Souza, A.V.G., Petretski, J.H., Demasi, M., Bechara, E.J.H., and Oliveira, P.L. 1996. Urate protects a blood-sucking insect against hemin-induced oxidative stress. Free Radical Biol. \& Med. 22:209-214.

Stellenwerf, W.A., Jr., and Hazelwood, R.L. 1979. Periperal utilization of a glucose load after alloxan and streptozotocin in the rat and chicken: a comparison. Gen. Comp. Endocrinol. 39:131-140.

Synder, D.L., 1989. Dietary restriction and aging. In: Proceedings of the Symposium on the effects of dietary restriction of aging and disease in Germfree and conventional lobund-Wistar rats. Prog. Clin. Biol. Res. 289. New York.

Thomas, C.E. and Reed, D.J. 1988. Effect of extracellular $\mathrm{Ca}^{++}$ommission on isolated hapatocytes. I. Introduction of oxidative stress and cell injury. J. Pharamacol. Exp. Ther. 245:493.

Thomas, C.E., and Reed, D.J. 1990. Radical-induced inactivation of kidney $\mathrm{Na}^{+}, \mathrm{K}^{+}$-ATPase: Sensitivity to membrane lipid peroxidation and the protective effect of vitamin E, Arch. Biochem. Biophys., 281:96.

Toumy, J.M., and Lechnir, R.J. 1964. Effect of cooking temperatures and time on the tenderness of beef. Food Technol. 17: 119-124.

Vaca, C.E., Whilhelm, J., and Harms-Ringdahl, M. 1988. Interaction of lipid peroxidation

products with DNA :A review. Mutant. Res 195:137-149.

Vandepopuliere and Lyons, J. J. 1996. Overview of spent hen alternatives. Proc. National poultry waste management. PP 113-118.

Van Rammen, J., Ward, W., Sabia, R.V., and Richardson, A. 1995. Gene expression and protein degradation. In Masoro, E.J., ed. Handbook of physiology, Sec. 11, aging. New York: oxford University Press :171-234.

Velasquez, M.T., Kimmel, P.L., and Michaelis, O.E, 1990. Animal models of spontaneously diabetic kidney disease. FASEB J 4:2850-2859. 
Vishwanath, V., Frank, D.E., Frank, D.E., Elmets, C.A., Dauchot, P.J., and Monnier, V.M. 1986. Glycation of skin collagen in type I diabetes mellitus: Correlation with long-term complications. Diabetes 35:916-921.

Vlassara, H., Brownlee, M., and Cerami, A. 1984a. Accumulation of diabetic rat peripheral nerve myelin by macrophages increases with the presence of advanced glycosylation endproducts. J. Exp. Med. 160: 197.

Vlassara, H., Brownlee, M., and Cerami, A. 1984b. High-affinity receptor-mediated uptake and degradation of glucose-mediated uptake and degradation of glucose-modified proteins: A potential mechanism for the removal of sencesecent macromolecules. Proc. Natn. Acad. Sci. USA 82: 5588.

Vlassara, H., Valinsky, J., Brownlee, M., Cerami, C., Nishimoto, S., and Cerami, A. 1987. Advanced glycosylation endproducts of the erythrocyte cell surface induce receptor-mediated phagocytosis by macrophages: a model for turnover of aging cells. J.exp. Med. 166: 539.

Vlassara, H., Brownlee, M., Manogue, K., Dinarello, C., and Pasagian, A., 1988. Cachectin/TNF and IL-1 induced by glucose-modified proteins: Role in normal tissue remodeling. Science 240:1546.

Vlassara, H., Bucala, R., and Striker, L. 1994. Pathogenic effects of advanced glycosylation: Biochemical, biologic, and clinical implications for diabetes and aging. Lab. Invest. 70: 38-151.

Vlassara, H., and Bucala, R. 1996. Recent progress in advanced glycation nd diabetic vascular disease: Role of advanced glycation endproduct receptors. Diabetes 45 (Suppl. 3):S65-S66.

Vater, C.A., Harris, Jr., E.D., and Siegel, R.C. 1979. Native crosslinks in collagen fibrils induce resistance to human synovial collagenase. Biochem. J. 181:639-645.

Verzar, F. 1963. The aging of collagen. Sci. Am. 208:104-114.

Voet, D., and Voet, J.G. 1990. Three-dimensional structure of proteins. In: Biochemistry. 1st ed. John Wiley \& Sons Inc. New York, NY.;144-190.

Weindruch, R., and Walford, R.L. 1982. Dietary restriction in mice beginning at 1 year of age: Effect of life-span and spontaneous cancer incidence. Science 215a;1415-1418.

Weindruch, R., and Walford, R.L. 1988. The retardation of aging and disease by dietary restriction. Springfield, IL Charles C Thomas.

Wess, T.J., Wess, L., Miller, A., Lindsay, R.M. and Baird, J.D. 1993. The in vivo glycation of diabetic tendon collagen studied by neutron diffraction. J. Mol. Biol. 230:1297-1303. 
Williamson, J.R., Rowold, E., Chang, K., Marvell, J., Tomlinson, M., Sherman,W.R., Ackermann, K.E., Berger, R.A., and Kilo, C. 1986. Sex steroid dependency of diabetes-induced changes in polyol metabolism, vscualar permeability, and collagen crosslinking. Diabetes $35: 20-27$.

Wolff, S.P., and Dean, R.T. 1987. Glucose autoxidation and protein modification: the potential role of autoxidative glycosylation in diabetes. Biochem J 245:253-250.

Wolff, S.P., Crabble, M.J.C., and Thornalley, P.J. 1987. The autoxidation of glyceraldehyde and other simple monosaccharides. Experientia 40:244.

Woods, K.L., Rhee, K.S. and Sams, A.R. 1997. Tenderizing spent fowl meat with calcium chloride. 4. Improved oxidative stability and the effects of additional aging. Poultry Sci. 76:548-551.

Wu, J. 1993. Advanced glycosylation end products: A new disease marker for diabetes and aging. J. Clin. Lab. Anal. 7:252-255

Yagi, K. 1991. Role of lipid peroxides in aging and age-related diseases. In: Ozawa, T., ed. New trends in biological chemistry. Berlin: springer Berlag; 1991; 207-223.

Yagihashi, S., M. Kamigo, M. Baba, N. Yahishi, and N. Nagai, 1992. Effect of aminoguanidine on functional and structural abnormalities in peripheral nerve of STZ-induced diabetic rats. Diabetes, 41:47-52.

Yamauchi, M., and Mechanic, G.L. 1987. Crosslinking of collagen. In: Nimni, M. E., ed. Collagen: vol. I. Biochemistry. Boca Raton, FL: CRC Press.

Yosa, S.F., Elden, F.R., Rabionoveitch, A., Mintz, D.H., and Boueckm R.J. 1983. Experimental diabetes mellitus and age-stimulated changes in intact rat dermal collagen. Diabetes 32:739-742.

Young, L.L., and Lyon, C.E. 1989. Effect of muscle pH and calcium content on quality of preand post-rigor chicken breast muscle. J. Food Sci. 54:1155-1157.

Young, L.L., and. Lyon, C.E., Kim, H., Papa, C.M., and Miller, M.F. 1989. Effect of varying calcium content on functional and textural properties of chicken breast muscle. Poultry Sci. $68: 212$.

Youngman, L.D., Park, J.Y., K., and Ames, B.N. 1992. Protein oxidation associated with aging is reduced by dietary restriction of protein or calories. Proc. Natl., Acad. Sci. 89:9112-9116. 
Youngman, L.D. 1993. Protein restriction (PR) and calorie restriction (CR)compared: Effects on DNA damage, carcinogenesis, and oxidative damage. Mutat. Res. 295:165-179.

Youngman, L.D., Park, J-YK., and Ames, B.N. 1994. Protein oxidation associated with aging is reduced by dietary restriction of proteins or calories. Proc. Natl. Acad. Sci. 1992;89:9112-9116.

Yu, B.P., Masoro, E.J., Murafa, I., Berfrand, H.Q and Lynd, F.T. 1982. Life-span study of SPF Fisher 344 male rats fed ad libitum or restricted diets: longevity, growth, lean body mass and disease. J. Gerontol.: Biol. Sci. 37:130.

Yu, B.P., Suescun, E.A., Yang, S.Y. 1992. Effect of age-related lipid peroxidation on lipid peroxidation on membrane fluidity and phospholipase A2: Modulation by dietary restriction. Mech. Ageing Dev. 65:17-33.

Yu, B.P. 1993. Need the free radical theory of aging be linked to the metabolic rate theory? Aging Clin. Exp. Res. 5:243-244.

Yu, B.P. 1993. Oxidative damage by free radicals and lipid peroxidation in aging. In: Yu, B.P., ed. Free radical in aging. Boca Raton, FL: CRC Press:57-88.

Yu, B.P. 1994. How diet influences aging process of the rat. Proc. Soc. Exp. Biol. Med. 205:97-105.

Yu, B.P. 1994a. Cellular defenses against damage from reactive oxygen species. Physiol. Rev. 74,139-162.

Yu, B. P. 1994b. How diet influences the aging process of the rat. Proc. Soc. Exp. Biol. Med. 205:97-107.

Yu, B.P. 1995. Putative intervention. In: Masoro, E.J., ed. Handbook of physiology, sect. 11. Aging. New York: Oxford University Press: P613-631.

Yu, B.P., Yang, Y. 1996. A critical evaluation of free radical theory of aging: Anew proposal of oxidative stress hypothesis. Ann. NY Acad. Sci. 786:1-11.

Yu, P.Y. 1996. Aging and oxidative stress: Modulation by dietary restriction. Free Radic.Biol. and Med. 21:651-668.

Yue, D.K., McLennan, S., Delbridge, L., Handlesman, D.J., Reeve, T., and Turtle, J.R. 1983. The thermal stability of collagen in diabetic rats: correlation with severity of diabetes and nonenzymatic glycosylation. Diabetologia 24:282-285. 
Yue, K.K., McLennan, S., Handlesman D.J., Delbridge, L., Reeve, T., and Turtle, J.r. 1984. The effect of salicylates on nonenzymatic glycosylation and thermal stability of collagen in diabetic rats. Diabetes 33:745-751.

Yue, K.K., McLennan, S., Handlesman D.J., Delbridge, L., Reeve, T., and Turtle, J.R. 1985. The effects of cyclooxygenase and lipoxygenase inhibitors on the collagen abnormalities of diabetic rats. Diabetes 34: 74-78.

Zs-Nay, I. 1994. The membrane hypothesis of aging. Boca Raton, FL: CRC Press. 